\title{
Vergleich der Radikalreaktionen von Melatonin und strukturverwandten Indolaminen in unterschiedlichen Oxidationssystemen
}

\author{
Dissertation \\ zur Erlangung des Doktorgrades \\ der Mathematisch-Naturwissenschaftlichen Fakultäten \\ der Georg-August-Universität zu Göttingen
}

\author{
vorgelegt von \\ Joachim Rosen \\ aus Lingen
}

Göttingen, den 18. September 2006 
D 7

Referent:

Korreferent:

Tag der mündlichen Prüfung:
Prof. Dr. Rüdiger Hardeland

Prof. Dr. Ralf Heinrich

02.11.2006 
Sic luceat lux 


\section{Inhaltsverzeichnis}

\section{Inhaltsverzeichnis}

\section{Abkürzungsverzeichnis}

1. Einleitung $\quad 1$

1.1 Kurze Geschichte des Melatonins 1

1.2 Biosynthese von Melatonin 2

1.3 Abbau von Melatonin 3

1.4 Ziel der Arbeit 7

$\begin{array}{ll}\text { 2. } & \text { Material und Methoden }\end{array}$

$2.1 \quad$ Geräte 8

$\begin{array}{lll}2.2 & \text { Chemikalien } & 10\end{array}$

$2.3 \quad$ Reaktionssysteme 11

2.3.1 Photokatalytischer Umsatz mit Protoporphyrin-IX 11

$\begin{array}{lll}2.3 .3 .1 & \text { Eichkurven } & 12\end{array}$

2.3.2 Hämin katalysiertes $\mathrm{H}_{2} \mathrm{O}_{2}$-System 14

$\begin{array}{lll}\text { 2.3.3 Oxidation mit ABTS-Kationradikalen } & 16\end{array}$

$\begin{array}{lll}2.4 & \text { Chromatographie } & 17\end{array}$

$\begin{array}{ll}\text { 2.4.1 Analytische Dünnschichtchromatographie } & 17\end{array}$

$\begin{array}{ll}2.4 .2 & 19\end{array}$

$\begin{array}{lll}2.5 & \text { Chemilumineszenzmessung } & 19\end{array}$

$\begin{array}{lll}2.6 & \text { Fluorometrie } 20\end{array}$

$\begin{array}{lll}2.7 & \text { Photometrie } & 21\end{array}$

$2.8 \quad$ Massenspektroskopie 21 
$2.9 \quad$ HPLC-MS

$2.10 \quad$ NMR

$2.11 \quad$ DBE-Wert

3. Ergebnisse

3.1 Photokatalytischer Umsatz von Indolen mit Protoporphyrin-IX

3.1.1 Umsatz von Melatonin mit Protoporphyrin-IX 23

3.1.2 Umsatz von 5-Methoxytryptamin mit Protoporphyrin-IX 25

3.1.3 Umsatz von $N$-Acetyltryptamin mit Protoporphyrin-IX 27

3.1.4 Umsatz von $N$-Acetylserotonin mit Protoporphyrin-IX 29

3.1.5 Umsatz von Serotonin mit Protoporphyrin-IX 31

3.1.6 Umsatz von Tryptamin mit Protoporphyrin-IX 33

3.2 Hämin katalysiertes $\mathrm{H}_{2} \mathrm{O}_{2}$-System 35

3.3 Oxidation mit ABTS-Kationradikalen 39

3.3.1 Umsatz von Melatonin mit ABTS- Kationradikalen 40

3.3.2 Umsatz von 5-Methoxytryptamin mit ABTS- Kationradikalen 41

3.3.3 Umsatz von $N$-Acetyltryptamin mit ABTS- Kationradikalen 42

3.3.4 Umsatz von $N$-Acetylserotonin mit ABTS- Kationradikalen 43

3.3.5 Umsatz von Serotonin mit ABTS- Kationradikalen 44

3.3.6 Umsatz von Tryptamin mit ABTS- Kationradikalen 45

3.4 Produkte der ABTS-Kationradikalreaktion 46

3.4.1 Produkt 2<smiles>CCC(=O)N/C=C1/C(=O)c2cc(OC)ccc2N1C=O</smiles>
49 
3.4.2 Produkt 3

3.4.2.1 Umsatzanalyse von Produkt 2 und 3

3.4.3 Produkt 4

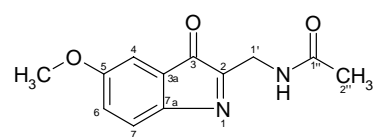

3.4.4 Produkt 5
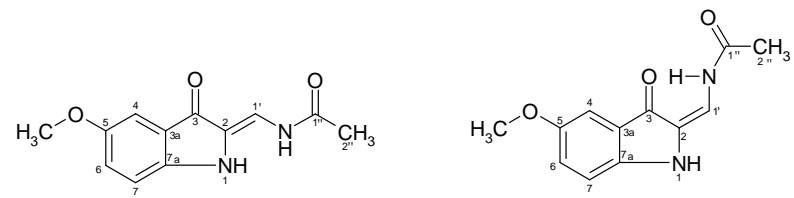

4. Diskussion

5. Zusammenfassung

6. Literaturverzeichnis 


\section{Abkürzungsverzeichnis}

5-MT

Abb.

ABTS

ABTS $^{\circ+}$

AFDK

AFMK

ALS

AMK

BEW

br

c3OHM

$\mathrm{CDCl}_{3}$

$\mathrm{CD}_{3} \mathrm{OD}$

CI-MS

COSY

$\mathrm{Cq}$

CYP

d

DBE

DCM

$\delta$

¿as

$\delta_{\mathrm{C}}$

$\delta_{\mathrm{H}}$

dd

dest. Wasser

DMSO

EDTA

EI-MS

ESI-HRMS

ESI-MS/MS
5-Methoxytryptamin

Abbildung

2,2'-Azino-bis(3-ethylbenzothiazolin-6-sulfonat)

2,2'-Azino-bis(3-ethylbenzothiazolin-6-sulfonat)-Kationradikal

$N^{1}$-Acetyl- $N^{2}$-formyl-5,6-dimethoxykynuramin

$N^{1}$-Acetyl- $N^{2}$-formyl-5-methoxykynuramin

amyotrophe Lateralsklerose

$N^{1}$-Acetyl-5-methoxykynuramin

Butanol, Eisessig, Wasser

breit

zyklisches 3-Hydroxymelatonin

Chloroform-d

Methanol-d

Chemical Ionization Mass Spectrometry

Correlated Spectroscopy

quaternäres Kohlenstoff-Atom

Cytochrom $\mathrm{P}_{450}$

Dublett

Double Bond Equivalence

Dichlormethan

Deformationsschwingung

asymmetrische Deformationsschwingung

chemische Verschiebung im ${ }^{13} \mathrm{C}-\mathrm{NMR}$ (in ppm relativ zu TMS)

chemische Verschiebung im ${ }^{1} \mathrm{H}-\mathrm{NMR}$ (in ppm relativ zu TMS)

Doppeldublett

destilliertes Wasser

Dimethylsulfoxid

Ethylendiamintetraessigsäure

Electron Ionization Mass Spectrometry

Electronspray Ionization High Resolution Mass Spectrometry

Electronspray Tandem Mass Spectrometry 


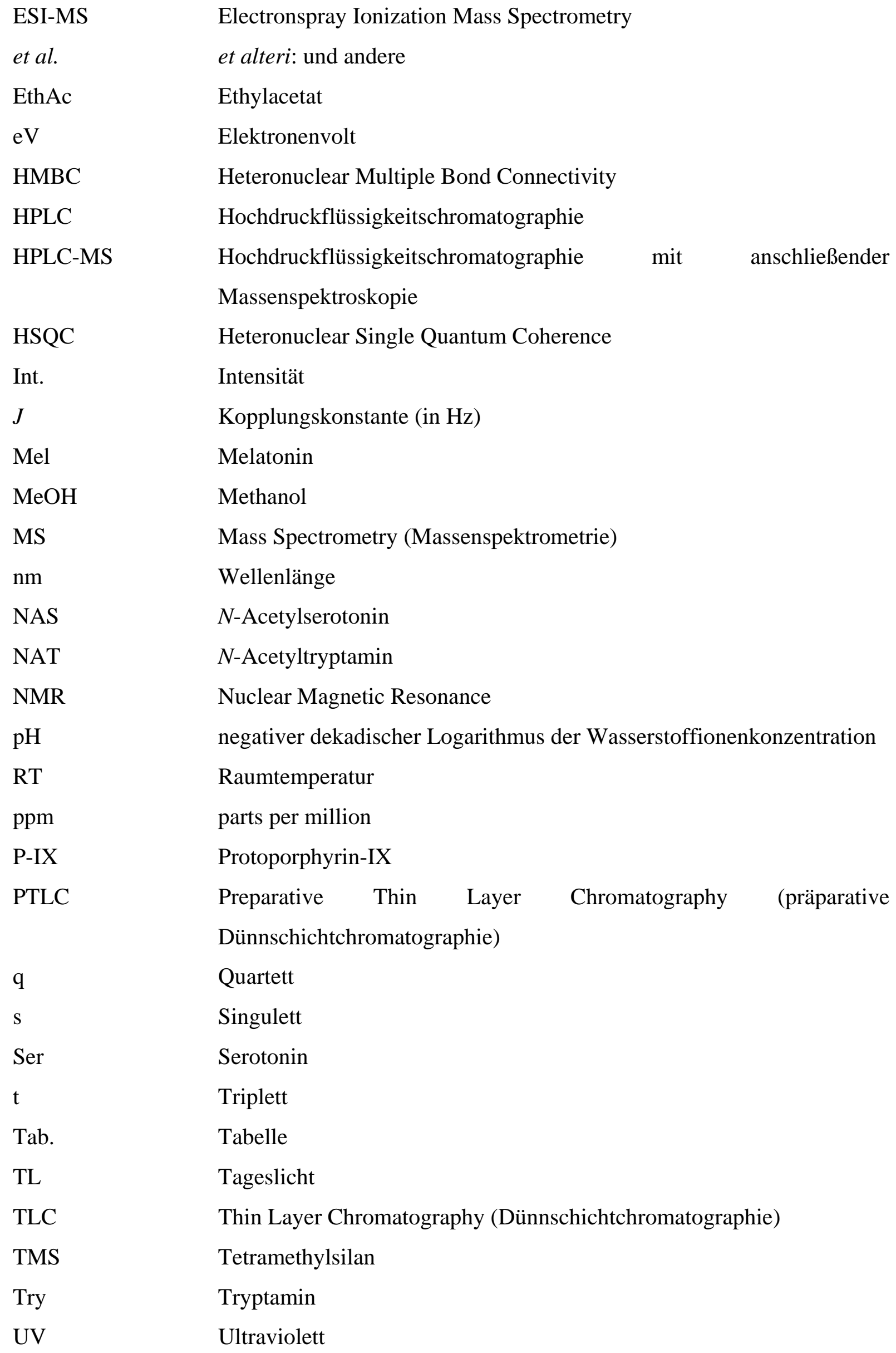




\section{Einleitung}

\subsection{Kurze Geschichte des Melatonins}

1917 entdeckten McCord und Allen, dass der Extrakt des Pinalorgans ${ }^{1}$, den sie Kaulquappen zu fressen gaben, zu einer dramatischen Aufhellung von deren Haut führte. 1958 schließlich gelang es Lerner und seinen Mitarbeitern (Lerner 1958), die Substanz aus dem Pinealorgan von Rindern zu isolieren und knapp ein Jahr später seine Struktur aufzuklären (Lerner 1959). Der Name Melatonin entstand aus der Zusammensetzung des Wortes melas (gr. für schwarz) und tonein ( $g r .$, im hier verwendeten Sinne: zusammenziehen) in Hinblick auf eine lokale Konzentrierung von Melanosomen in den Melanocyten.

Zunächst glaubte man, dass das Neurohormon Melatonin nur bei Vertebraten vorkommen würde (Reiter, 1991; Tan et al., 2003). Spätere Untersuchungen zeigten jedoch, daß es nahezu in allen Lebensformen, wie z.B. Bakterien, Pilzen, Mikro- und Makroalgen, Kormophyten und Invertebraten wieder zu finden ist (Poeggeler et al., 1991; Hardeland \& Fuhrberg, 1996; Hardeland, 1999; Manchester et al., 2000; Reiter et al., 2001; Hardeland \& Poeggeler, 2003; Hardeland \& Pandi-Perumal, 2005).

Die Konzentrationen von Melatonin im Blut von Vertebraten, einschließlich des Menschen, zeigen einen ausgeprägten circadianen Rhythmus. Die Produktion im Pinealorgan erfolgt ganz überwiegend in der Nacht, was bei Änderungen der Nachtlänge zu saisonalen Unterschieden führt. Bei Kindern kann die Konzentration in der Nacht bis zu 300 pg/mL betragen (Waldhauser et al., 1993; Karasek et al., 2004). Bei Jugendlichen beträgt sie noch 10-40 $\mathrm{pg} / \mathrm{mL}$ am Tag und 60-120 pg/mL in der Nacht. Mit zunehmenden Alter sinkt der Wert auf Nachtwert zwischen 20-50 pg/mL (Karasek et al., 2004).

\footnotetext{
${ }^{1}$ Weitere Bezeichnungen: Epiphyse, Zirbeldrüse, Glandula Pinealis, Corpus Pineale, Epiphysis Cerebri
} 


\section{$\underline{1.2}$ Biosynthese von Melatonin}

Die Biosynthese des Melatonins beginnt mit Tryptophan und geht über die Zwischenstufen 5-Hydroxytryptophan, 5-Hydroxytryptamin (Serotonin) und $\mathrm{N}$-Acetylserotonin. Die entscheidenden Enzyme sind hierbei die Arylalkylamin- $N$-acetyltransferase und die 5-Hydroxyindol-O-methyltransferase (Illnerova \& Vanecek, 1980; Erlich \& Apuzzo, 1985; Ebadi \& Govitrapong, 1986; Reiter, 1987; Illnerova, 1988; Sugden, 1989). Melatonin kann ebenfalls aus Methoxytryptamin durch die Arylalkylamin- $N$-acetyltransferase gebildet werden (Erlich \& Apuzzo, 1985) (Abb. 1).<smiles>NC(Cc1c[nH]c2ccc(O)cc12)C(=O)O</smiles>

Tryptophan

5-Hydroxytryptophan<smiles>CC(=O)NCCc1c[nH]c2ccc(O)cc12</smiles>

$N$-Acetylserotonin

5-HydroxyindolO-methyl-

Arylalkylamin$\mathrm{N}$-acetyltransferase<smiles>NCCc1c[nH]c2ccc(O)cc12</smiles>

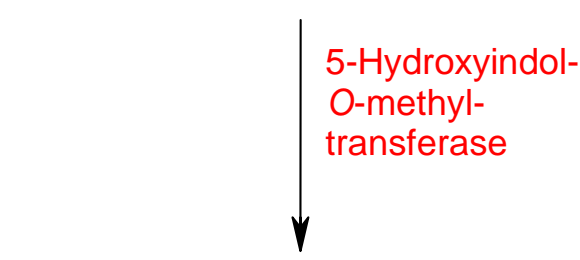

\section{Serotonin} 5-HydroxyindolO-methyltransferase<smiles>COc1ccc2[nH]cc(CCN)c2c1</smiles>

Melatonin

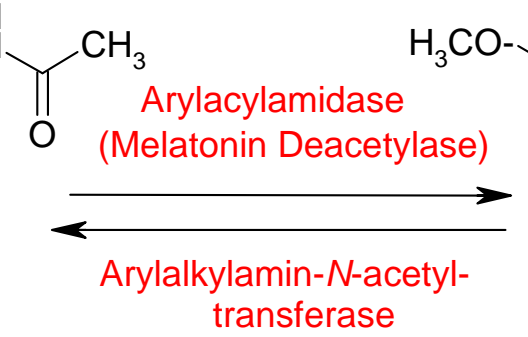<smiles>Cc1ccc2[nH]cc(CCN)c2c1</smiles>

5-Methoxytryptamin transferase

Abb. 1: Biosynthese des Melatonins 


\section{$\underline{1.3 \text { Abbau von Melatonin }}$}

Die klassische Vorstellung vom Abbau des Melatonins geht von einem hohen „first-pass“Effekt aus. Zwischen 92-95 \% des zirkulierenden Melatonins werden bereits während der ersten Leberpassage aus dem Blut entfernt (Pardrigde \& Mietus, 1980). Hierbei wird durch Cytochrom $\mathrm{P}_{450}$-Monoxygenase 6-Hydroxymelatonin (Abb. 2) gebildet, welches anschließend zu 6-Sulfoxymelatonin konjugiert werden kann (Kopin et al., 1961). In dieser Form ist es sehr hydrophil und wird mit dem Urin ausgeschieden.

Diese Sichtweise mag für das zirkulierende Melatonin zutreffen, nicht jedoch notwendigerweise für das Gewebemelatonin. Es sei darauf hingewiesen, daß z. B. der Gastrointestinaltrakt ein hundertfaches dessen an Melatonin enthält wie das Pinealorgan (Bubenik, 2001; 2002). Der Metabolismus muß in einem solchen Organ schon aufgrund anderer Enzymausstattung verschieden sein. In Teilen des Gehirns, insbesondere der Retina, wird Melatonin größtenteils zu 5-Methoxytryptamin deacetyliert, wobei eine spezifische Melatonindeacetylase oder unspezifische Arylacylamidase ebenso wie Acetylcholinesterase (Grace et al., 1993) diese Reaktion katalysieren.

Ein weiterer Abbauweg besteht in der Spaltung des Pyrrolringes, was zur Bildung eines substituierten Kynuramins führt, $N^{1}$-Acetyl- $N^{2}$-formyl-5-methoxykynuramin (AFMK; Abb. 2). Dieses kann weiter deformyliert werden zu $N^{1}$-Acetyl-5-methoxykynuramin (AMK; Abb. 2). Bereits bei der Entdeckung dieser Substanzen (Hirata et al., 1974) wurde gezeigt, daß sie Hauptmetaboliten des Melatonins im Zentralnervensystem darstellen, wobei 6-Hydroxymelatonin unter der Nachweisgrenze blieb. Inzwischen sind diverse Wege der AFMK-Bildung gefunden worden (Tab. 1). Neuere Schätzungen sind zu dem Ergebnis gekommen, daß ca. 1/3 des gesamten Melatonins im Körper in diesen Abbauweg gehen (Ferry et al., 2005).

Bildung und weitere Verstoffwechselung von AFMK sind demnach für das Verständnis der Funktion des Melatonins relevant, insbesondere als auch AFMK und AMK biologische Aktivität entfalten (Martinez et al., 2005).

Die Beachtung des Gewebemelatonins ist unter mehreren Gesichtspunkten von Bedeutung. Aufgrund seiner Amphiphilie gelangt Melatonin in alle Zellen und Zellkompartimente. 
Dort finden in erhöhten Maße Radikalreaktionen statt, die zur Metabolisierung des Melatonins beitragen (vgl. Tab. 1)<smiles>CC(=O)NCCc1c[nH]c2ccc(O)cc12</smiles>

$\mathrm{N}$-Acetylserotonin

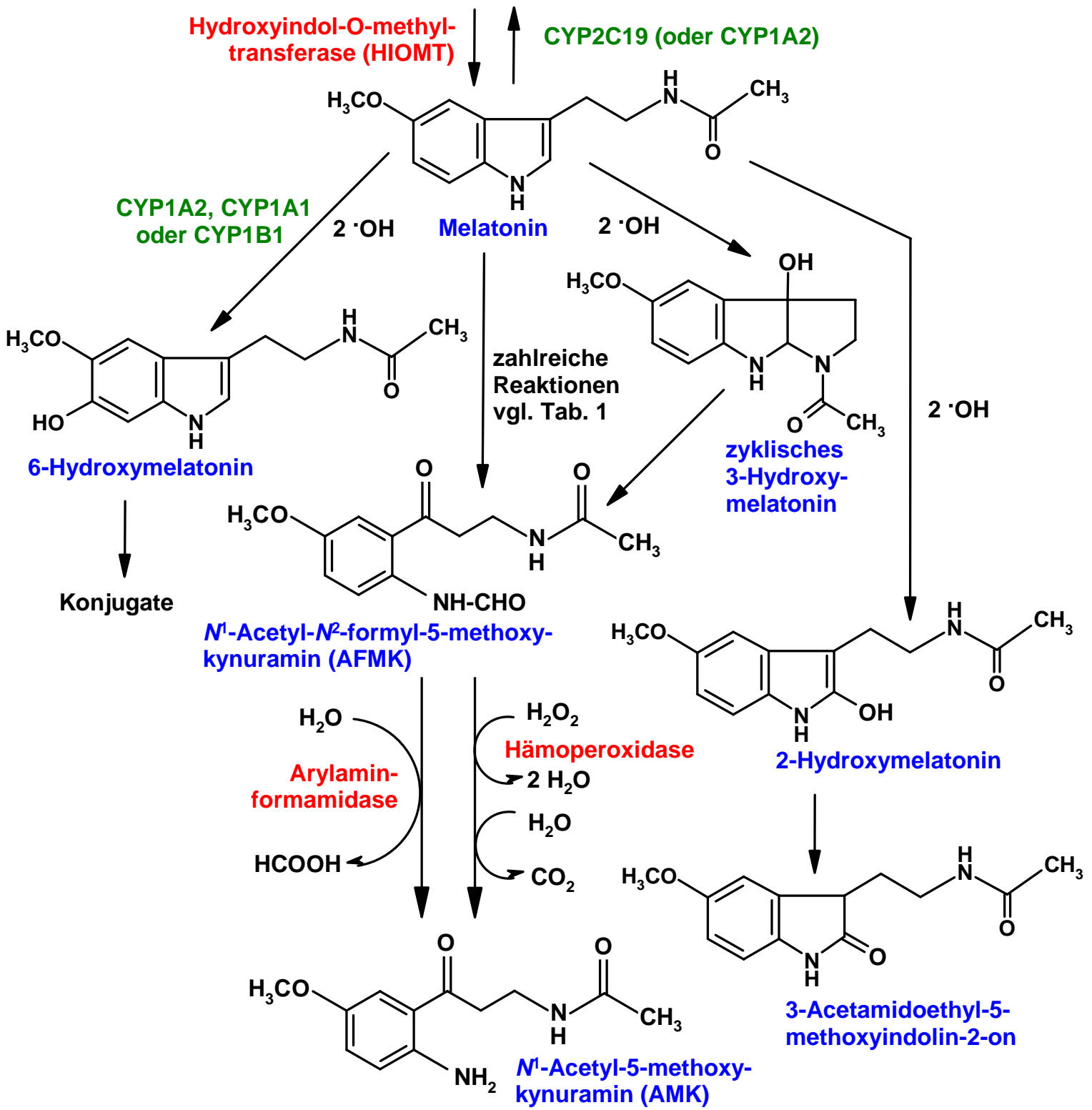

Abb. 2: Metabolisierungswege von Melatonin 
Tab. 1: Mechanismen der AFMK-Bildung

\begin{tabular}{|c|c|}
\hline Katalysatoren und Reaktanden & Referenzen \\
\hline \multicolumn{2}{|l|}{ A) Enzymatische Mechanismen } \\
\hline $\begin{array}{l}\text { Indolamin-2,3-dioxygenase; } \mathrm{O}_{2}{ }^{\circ-} \text { als } \\
\text { Cosubstrat. } \\
\text { Hämoperoxidase (Meerrettich)/Compound } \\
\text { III, Involvierung von } \mathrm{O}_{2}{ }^{--} \\
\text {Myeloperoxidase; } \mathrm{H}_{2} \mathrm{O}_{2} \text {. }\end{array}$ & $\begin{array}{l}\text { Hayaishi, 1976; Hardeland et al., 1993c; } \\
\text { Hardeland \& Fuhrberg, } 1996 . \\
\text { Ximenes et al., 2001. } \\
\text { Ximenes et al., 2001; Rodrígues et al., } 2003 .\end{array}$ \\
\hline
\end{tabular}

\section{B) Pseudoenzymatische Mechanismen}

\begin{tabular}{|c|c|}
\hline Hämin; $\mathrm{O}_{2}{ }^{--}$ & $\begin{array}{l}\text { Fuhrberg \& Hardeland, 1994; Hardeland \& } \\
\text { Fuhrberg, 1996; Hardeland et al., 1993a, } \\
\text { 1993c; Poeggeler et al., 2002; Hardeland } \\
\text { \& Poeggeler, 2002. }\end{array}$ \\
\hline $\begin{array}{l}\text { Oxoferryl-Hämoglobin (aus Methämoglobin } \\
\text { und } \mathrm{H}_{2} \mathrm{O}_{2} \text { ). }\end{array}$ & Tesoriere et al., 2001. \\
\hline
\end{tabular}

\section{C) Photokatalytische Mechanismen}

\begin{tabular}{|l|l|}
\hline Protoporphyrinyl-Kationradikale; $\mathrm{O}_{2}{ }^{--}$ & Uría et al., 1994, 1995; Hardeland et al., \\
& 1995, 1996; Hardeland \& Fuhrberg, 1996; \\
& Burkhardt et al., 2001a. \\
& Tomás-Zapico et al., 2001. \\
Anthranilyl-Radikale; $\mathrm{O}_{2}{ }^{--}$ & Behrmann, 1989; Hardeland et al., 1991, \\
Extrakte aus Dinoflagellaten; $\mathrm{O}_{2}{ }^{--}$ & 1993b, 1995, 1996; Hardeland \& \\
& Poeggeler, 1995. \\
\hline $\begin{array}{l}\text { Extrakte aus Phaeophyceen; } \mathrm{O}_{2}{ }^{-*} \cdot \\
\text { Chlorophyll a (unter vorzugsweiser Bildung } \\
\text { anderer Produkte). } \\
\text { Singulettsauerstoff (mit geringer Effizienz). }\end{array}$ & Hardeland, 1996. \\
\hline
\end{tabular}

\section{D) Photoexzitatorische Mechanismen}

Sichtbares Licht; $\mathrm{O}_{2}$.

UV (in wässriger Lösung oder auf

Trockensubstanz);

$\mathrm{O}_{2}$.
Burkhardt et al., 2001a; Tan et al., 2001;

Tan, 2002.

Behrmann et al., 1995; Burkhardt et al., 2001a.

\section{E) Ozonolyse}

Ozon.

Hardeland \& Fuhrberg, 1996; Hardeland, 1997. 


\begin{tabular}{|c|c|}
\hline $\begin{array}{l}\text { F) Radikalische Mechanismen } \\
\text { (außerhalb der schon zuvor erwähnter } \\
\text { photokatalytischen Mechanismen) }\end{array}$ & enzymatischen, pseudoenzymatischen oder \\
\hline $\begin{array}{l}\begin{array}{l}\text { Elektronen-Abstraktion durch }{ }^{\circ} \mathrm{OH} \text { und } \\
\text { nachfolgende Kombination mit } \mathrm{O}_{2}{ }^{\cdot-}\end{array} \\
\begin{array}{l}\text { Elektronen-Abstraktion durch } \mathrm{CO}_{3}^{\cdot-} \\
\text { nachfolgende Kombination mit } \mathrm{O}_{2}{ }^{\cdot-}\end{array}\end{array}$ & $\begin{array}{l}\text { Hardeland \& Fuhrberg, 1996; Hardeland et } \\
\text { al., 1993c, 1996; Burkhardt et al., 2001a; } \\
\text { Tan et al., 2001, 2002; Poeggeler et al., } \\
\text { 2002; Hardeland \& Poeggeler, 2002; } \\
\text { Schoenke et al., 2001. } \\
\text { Hardeland et al., 2001, 2003c. }\end{array}$ \\
\hline
\end{tabular}

G) Radikalische Reaktionen des cyclischen 3-Hydroxymelatonins ( c-3OHM)

\begin{tabular}{l|l} 
Oxidation von c3OHM durch & Tan, 2002.
\end{tabular}

elektronenabstrahierende Radikale in

Gegenwart von Wasser.

Unter praktischen Aspekten sind diese Zusammenhänge auch deswegen von Interesse, weil Melatonin als Medikament (u.a. gegen Jetlag, amyotrophe Lateralsklerose (ALS), Schlafstörungen) und als Nahrungsergänzungsstoff weltweit eingesetzt wird.

Eine besondere und derzeit viel beachtete Wirkung des Melatonins betrifft die antioxidative Protektion (Hardeland et al., 2003a), mit dem Teilaspekt des Radikalfangens. Hierbei stellt sich die Frage, was das Melatonin von anderen Indolaminen diesbezüglich unterscheidet. Dass in dieser Hinsicht beträchtliche Divergenzen in der Redox-Chemie bestehen, hatte sich bereits in früheren Studien gezeigt (Poeggeler et al., 2002), die jedoch nicht ausführliche Strukturuntersuchungen der Produkte beinhaltet hatte. 


\subsection{Ziel der Arbeit}

Ziel dieser Arbeit war die Untersuchung verschiedener Indole (Tryptamin, 5-Methoxytryptamin, $N$-Acetyltryptamin, $N$-Acetylserotonin, Serotonin, Melatonin und 6-Methoxymelatonin, Abb. 3) in Reaktionen mit unterschiedlichsten Radikalsystemen.

Da auch die Produkte der Radikalreaktionen von potentieller Bedeutung sind, lag es nahe, auch deren Redox-Verhalten einzubeziehen. Dies betraf insbesondere das AFMK, von dem bislang zwar bekannt war, daß es Hydroxylradikale zu fangen imstande ist (Burkhardt et al., 2002, Tan et al. 2001), von dem aber keine radikalisch erzeugten Folgeprodukte bekannt waren.<smiles>CC(=O)NCCc1c[nH]c2ccccc12</smiles><smiles>CC(=O)OCCOC(C)(C)C</smiles><smiles>COc1ccc2[nH]cc(CCNC(C)=O)c2c1</smiles><smiles>CCOCCOCCOCC</smiles><smiles>NCCc1c[nH]c2ccc(O)cc12</smiles><smiles>NCCc1c[nH]c2ccccc12</smiles> 


\section{Material und Methoden}

\subsection{Geräte:}

Folgende Materialien und Geräte wurden im Rahmen dieser Arbeit verwendet:

- Aquariumpumpe: Wisa, Typ 301.000.010.0

- Chromatographiesäule: Für Sephadex LH 20; Gesamthöhe der Säule: 64,0 cm; Höhe vom Sephadex in der Säule: 49 cm; Durchmesser der Säule: 1,5 cm

- Dünnschichtchromatographie-Alufolien: Merck, Darmstadt, Deutschland, 20x20 cm², Kieselgel $60 \mathrm{~F}_{254}$

- Fertigfolien für Dünnschichtchromatographie: Merck, Darmstadt, Deutschland, 40x80 $\mathrm{mm}^{2}$

- Fritten: Schott Duran, Mainz, Deutschland

- Kieselgel (a): Aldrich, Milwaukee, USA, TLC high-purity grade, ohne Binder, Partikelgröße 5-25 $\mu$, Oberfläche $\sim 500 \mathrm{~m}^{2} / \mathrm{g}$

- Kieselgel (b) für die Säulenchromatographie: Macherey-Nagel, Düren, Deutschland, MN Kieselgel 60, 0,05-0,2 mm / 70-270 mesh ASTU, für die Säulen-Chromatographie

- Kieselgel (c) für die präparative Dünnschichtchromatographie: Macherey-Nagel, Düren, Deutschland, MN Kieselgel P/UV254

- Kristallisationsschalen: Schott Duran, Mainz, Deutschland, mit Ausguss, Durchmesser 50 mm, Höhe 30 mm, Volumen 40 mL

- Leuchstoffröhre: Osram $20 \mathrm{~W}$, Hellweiß, Photonenflußrate $130 \mu \mathrm{M}$ Photonen* $\mathrm{m}^{-2} \mathrm{~s}^{-1}$

- Mixer: Heidolph, Typ 50110 RZR-O, 280-2200 U/min

- pH-Meter: Knick, Digital-pH Meter (bezogen über E. Schütt jr., Göttingen, Deutschland)

- Reagenzgläser von Fiolax: Schott, Mainz, Deutschland, mit Bördelrand, dünnwandig, Außendurchmesser 12 mm, Länge 100 mm, Wanddicke 0,4-0,5 mm

- Rotationsverdampfer: Büchi Laboratoriums-Technik, Flawil, Schweiz, Rotavapor Typ RE-111

- Rückflusskühler: Krannich, Göttingen, Deutschland

- Schüttler: GFL, Burgwedel, Deutschland, Typ 3016

- Sephadex LH-20: Fluka, Buchs, Schweiz, Teilchengröße 25-100 $\mu \mathrm{m}$ 
- Simultan-Trennkammer mit Knopfdeckel: DESAGA, Wiesloch, Deutschland, $200 \times 200 \mathrm{~mm}^{2}$

- Spektralfluorometer: Aminco-Bowman, Nr. JA-8965-E, mit ellipsoidalem Kondensationssystem, temperiert durch Colora Typ WK-3 Messtechnik GmbH, Lorch/Württemberg, Deutschland

- $\quad$ Spektralphotometer (a): Zeiss, Deutschland, PM7

- Spektralphotometer (b): Biochrom, Cambridge, England, Ultrospec II, Modell 4050

- Szintillations-Fläschchen: Neolab, Heidelberg, Deutschland, Wheaton-SzintillationsFläschchen, $20 \mathrm{~mL}$

- Szintillationszähler: Packard, Modell 3330

- Tischzentrifuge: Hettich EBA 35

- Ultraschallbad: Bandelin, Berlin, Sonorex RK 510 Transistor

- UV-Lampe (a): DESAGA, Heidelberg, Deutschland, mit Röhren der Emissionswellenlängen $254 \mathrm{~nm}$ und $366 \mathrm{~nm}$

- UV-Lampe (b): Lamag, CAT. No. 29010, SER. No. 881042, mit Röhren der Emissionswellenlängen $254 \mathrm{~nm}$ und $366 \mathrm{~nm}$

- Vakuumpumpe: Brand, Wertheim/Main, Deutschland, Drehschieber-Vakuumpumpe, Typ RD-4

- Ventilator: Salco, Tisch-Ventilator, Modell KB-307A

- Wasserbad: Julabo Labortechnik, Seelbach, Julabo EM/3

- Zentrifuge: Eppendorf, Deutschland, Eppendorf-Laborfuge 5417C

- $\quad$ EI-MS: Finnigan MAT 95 (70 eV)

- ESI-MS: Finnigan LCQ

- HPLC-MS: HPLC: Quarternäre HPLC-Pumpe (Flux Instruments), Autosampler (Jasco), UV-Detektor; MS: Finnigan LCQ

- HRESI-MS: Apex IV 75-FT-ICR-MS (Bruker Daltonik)

- ${ }^{1}$ H-NMR-Spektren: Varian Inova 600 (599,7 MHz), Varian Mercury 300 (300,1 MHz), Varian Unity 300 (300,1 MHz)

- ${ }^{13}$ C-NMR-Spektren: Varian Mercury 300 (75,5 MHz), Varian Inova 600 (150,8 MHz), Varian Unity 200 (50,3 MHz), Varian Unity 300 (75,5 MHz) 


\subsection{Chemikalien:}

Die verwendeten Chemikalien wurden aus folgenden Quellen bezogen:

Fluka, Buchs, Schweiz:

\section{ABTS}

Sigma-Aldrich, Steinheim, Deutschland:

Tryptamin, 5-Methoxytryptamin, 5-Hydroxytryptamin (Serotonin), $\mathrm{N}$-Acetyltryptamin, $\mathrm{N}$-Acetylserotonin, Kaliumpersulfat, Melatonin, Protoporphyrin-IX,

Alle weiteren Chemikalien stammten von der Firma Merck, Darmstadt, Deutschland:

Das 6-Methoxymelatonin wurde freundlicherweise von Dr. Russel J. Reiter vom Health Science Center der Universität von Texas in San Antonio zur Verfügung gestellt.

\section{Herstellung von AFMK:}

Das in dieser Arbeit verwendete AFMK wurde in der Arbeitgruppe von Prof. Dr. H. Laatsch im Institut für Organische und Biomolekulare Chemie der Georg-August-Universität Göttingen hergestellt. Die Synthese erfolgte aus Melatonin nach der Methode von Kennaway et al. (1988).

\section{Herstellung von ABTS-Kationradikalen $\left(\mathrm{ABTS}^{\bullet+}\right)$ :}

Stammlösung $50 \mathrm{~mL}$

Reaktionsansatz: $\quad$ Endkonzentrationen:
$192,500 \mathrm{mg}$
ABTS
$(7,00 \mathrm{mM})$
33,075 mg
Kaliumpersulfat $(2,45 \mathrm{mM})$

Der Reaktionsansatz wurde in einem mit Alufolie umwickelten Falcon-Röhrchen mit 50 mL Kalium-Phosphat-Puffer $50 \mathrm{mM}(\mathrm{pH} \mathrm{7,5)}$ angesetzt. Die Lösung wurde $15 \mathrm{~min}$ lang geschüttelt und bis zur endgültigen Gebrauchsfähigkeit bei RT 16 Stunden im Dunkeln aufbewahrt. Hiernach betrug die Konzentration von $\operatorname{ABTS}^{\bullet+}$ ca. 4,2 mol/L.

Kühlgelagert war die Lösung mehrere Tage lang stabil. Für verschiedene Reaktionsansätze wurden andere $\mathrm{ABTS}^{\bullet+}$ Konzentrationen benötigt. Hierzu wurde die gebrauchsfähige Lösung entsprechend mit Kalium-Phosphat-Puffer ( $\mathrm{pH}$ 7,5) anhand des molaren Extinktionskoeffizienten von $36000 \mathrm{M}^{-1} \mathrm{~cm}^{-1}$ bei $420 \mathrm{~nm}$ verdünnt. 


\section{Herstellung von präparativen Dünnschichtchromatographie-Platten}

Mit Hilfe eines Rührers wurde eine Aufschwemmung von 660 g Kieselgel in 1 L dest. Wasser hergestellt. Je $60 \mathrm{~mL}$ der Aufschwemmung wurden auf eine Glasplatte $\left(20 \times 20 \mathrm{~cm}^{2}\right)$ gegossen und gleichmäßig verteilt. Die Platten wurden für einen Tag an der Luft zum Trocknen liegen gelassen und am nächsten Tag für $3 \mathrm{~h}$ bei $120^{\circ} \mathrm{C}$ aktiviert.

\section{Herstellung von Chromatographiesäulen}

Das Trägermaterial Sephadex (siehe Chemikalien) wurde je nach Verwendung mit dem entsprechenden Laufmittel versetzt und vorsichtig in die Säule (siehe Geräteliste) eingefüllt. Durch mehrfaches Auffüllen und wieder Ablassen des Laufmittels wurde die Säule gespült, bis sich die stationäre Phase gesetzt hatte.

Vor Benutzung der Säule wurde die mobile Phase so weit abgelassen, bis sie sich kurz über der stationären Phase befand.

\section{$\underline{2.3 \text { Reaktionssysteme: }}$}

\subsubsection{Photokatalytischer Umsatz mit Protoporphyrin-IX}

Die Indole wurden in ethanolischer Lösung mit dem Photooxidans Protoporphyrin-IX unter Belichtung mit einem Photonenfluß von $130 \mu \mathrm{M}$ Photonen $\bullet \mathrm{m}^{-2} \cdot \mathrm{s}^{-1}$ umgesetzt

Reaktionsansatz:

$200 \mu \mathrm{L}$ Indol in EtOH $^{*}$

$400 \mu \mathrm{L}$ Protoporphyrin-IX in EtOH $400 \mu \mathrm{L}$ Ethanol

* folgende Indole wurden mit diesem Ansatz getestet: Melatonin

\section{Endkonzentrationen:}

$$
\text { ( } 20 \mathrm{mM}) \quad 4,00 \mathrm{mM}
$$$$
(100 \mu \mathrm{M}) \quad 0,04 \mathrm{mM}
$$

Tryptamin, 5-Methoxytryptamin, $N$-Acetyltryptamin, $N$-Acetylserotonin, Serotonin und 


\subsubsection{Eichkurven}

Umsatzberechnungen wurden an Hand von zuvor erstellen Eichkurven durchgeführt.
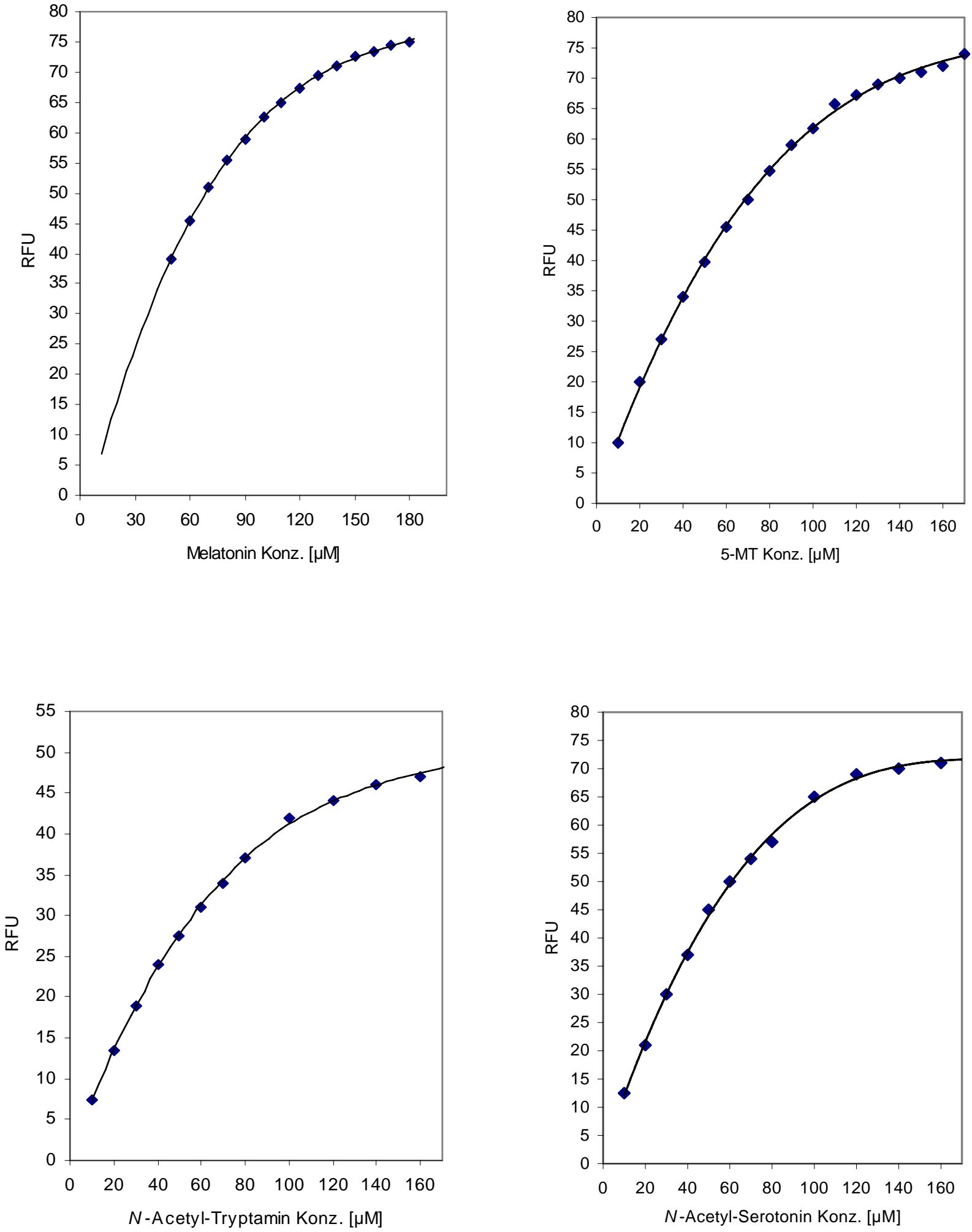

Abb. 4a. Eichkurven von Melatonin, 5-MT, NAT, NAS

Seite 12 

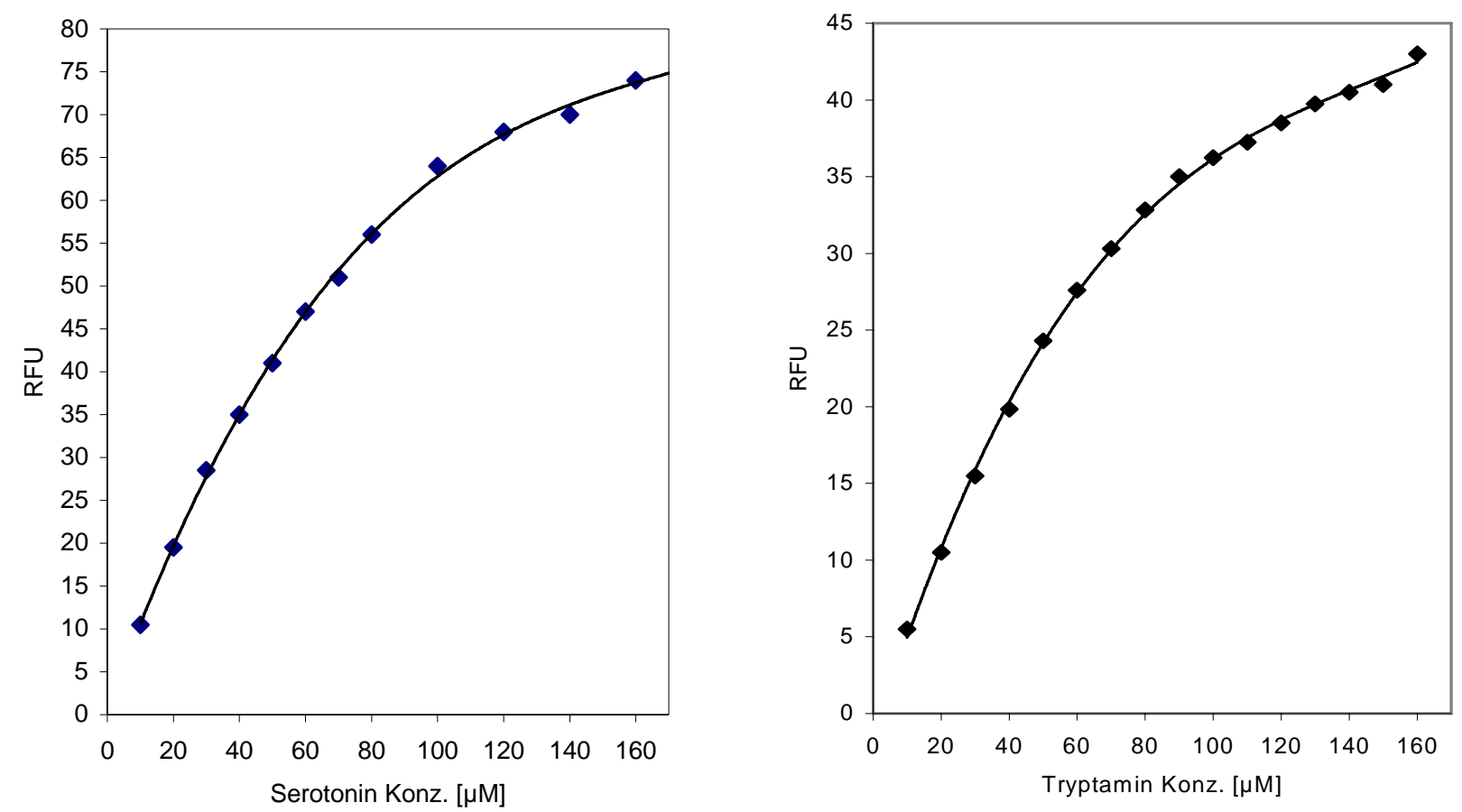

Abb. 4b. Eichkurven von Serotonin und Tryptamin

Zur Ermittlung der Konzentration in Abhängigkeit von der relativen Fluoreszenz wurden die verschiedenen Indole in ethanolischen Lösungen ihren Excitations- und Emissionsmaxima entsprechend gemessen.

Melatonin: Ex=350 / Em=285; 5-MT: Ex: 284 / Em=340; NAT: Ex=281 / Em=360; NAS: Ex=290 / Em=350; Serotonin: Ex=290 / Em=340; Tryptamin: Ex=275 / Em=356. 


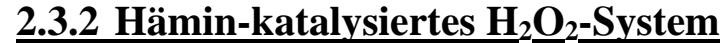

In diesem Assay nach Fuhrberg \& Hardeland (1994) verläuft die Entstehung der Radikale nicht „burst“-artig wie in Systemen, in denen die Fenton-Reaktion mit freiem Eisen statt findet, sondern langsamer und mit einer konstanteren Rate. Hierbei werden aus dem alkalischen $\mathrm{H}_{2} \mathrm{O}_{2}$-System Superoxidanion- und Hydroxylradikale gebildet. In diesem Reaktionssystem laufen, neben anderen, zwei wichtige Reaktionen ab, welche das Charakteristikum dieses Assays darstellen. Im alkalischen Milieu ist die Bildung von Superoxidanionen begünstigt, weil der Entzug von Protonen aus dem Gleichgewicht:

$$
\mathrm{H}_{2} \mathrm{O}_{2}+\mathrm{O}_{2} \rightarrow 2 \mathrm{H}^{+}+2 \mathrm{O}_{2}{ }^{-}
$$

die Nachbildung von Superoxidanionen treibt.

Hämin vermag eine Oxidation durch Übertragung von molekularem Sauerstoff aus Superoxidanionen zu katalysieren, wobei es selber reduziert wird (Hardeland \& Fuhrberg, 1996).

Desweiteren läuft eine Fenton-Reaktion ab:

$$
\begin{aligned}
& \mathrm{Fe}^{3+} \text {-Komplex }+\mathrm{O}_{2} \bullet^{-} \rightarrow \mathrm{Fe}^{2+}-\text { Komplex }+\mathrm{O}_{2} \\
& \text { (alternativ kann auch eine Reduktion durch } \mathrm{H}_{2} \mathrm{O}_{2} \text { erfolgen) } \\
& \mathrm{Fe}^{2+} \text {-Komplex }+\mathrm{H}_{2} \mathrm{O}_{2} \rightarrow \mathrm{Fe}^{3+}-\text { Komplex }+\cdot \mathrm{OH}+\mathrm{OH}^{-}
\end{aligned}
$$

\section{Reaktionsansatz:}

Zur Prüfung auf die Anteile $\bullet \cdot \mathrm{OH}$ - und $\mathrm{O}_{2}{ }^{\bullet^{-}}$-abhängigen Reaktionen wurden folgende Varianten mit DMSO als • OH-Fänger und Tiron als $\mathrm{O}_{2}{ }^{-}$-Fänger untersucht.

Reaktionsansatz:

Endkonzentrationen:

0,7 mL Gly-Gly-Puffer pH $8.0(100 \mathrm{mM}) \quad 60 \mathrm{mM}$

0,1 mL 6-MeO-Mel (4 mM)* in Puffer $\quad 400 \mu \mathrm{M}$

$0,1 \mathrm{~mL} \quad \mathrm{Hämin}-\mathrm{Cl}(50 \mu \mathrm{M}) \quad 5 \mu \mathrm{M}$

$0,1 \mathrm{~mL} \quad \mathrm{H}_{2} \mathrm{O}_{2} 10 \% \quad 1 \%$

- Inkubation für 1 Stunde bei RT im Szintillationszähler

0,77 mg 6-MeO-Mel wurden in $20 \mu \mathrm{l}$ DMSO vorgelöst 
$\underline{\text { Reaktionsansatz und zusätzliches Tiron als } \mathrm{O}_{2}} \underline{ }^{{ }^{-} \text {-Fänger }}$

Reaktionsansatz:

Endkonzentrationen:

0,6 mL Gly-Gly-Puffer pH $8.0(100 \mathrm{mM}) \quad 60 \mathrm{mM}$

$0,1 \mathrm{~mL}$ Tiron $(20 \mathrm{mM})$ in Puffer $\quad 400 \mu \mathrm{M}$

0,1 mL 6-MeO-Mel (4 mM)* in Puffer $\quad 400 \mu \mathrm{M}$

$0,1 \mathrm{~mL} \quad$ Hämin-Cl $(50 \mu \mathrm{M}) \quad 5 \mu \mathrm{M}$

$0,1 \mathrm{~mL} \quad \mathrm{H}_{2} \mathrm{O}_{2} 10 \% \quad 1 \%$

- Inkubation für 1 Stunde bei RT im Szintillationszähler

$\underline{\text { Reaktionsansatz und zusätzliches DMSO als •OH-Fänger }}$

Reaktionsansatz: Endkonzentrationen:

0,6 mL Gly-Gly-Puffer pH 8.0 (100 mM) $60 \mathrm{mM}$

$0,1 \mathrm{~mL} \quad$ DMSO

$0,1 \mathrm{~mL} \quad$ 6-MeO-Mel $(4 \mathrm{mM})^{*}$ in Puffer $\quad 400 \mu \mathrm{M}$

$0,1 \mathrm{~mL} \quad$ Hämin-Cl $(50 \mu \mathrm{M}) \quad 5 \mu \mathrm{M}$

$0,1 \mathrm{~mL} \quad \mathrm{H}_{2} \mathrm{O}_{2} 10 \% \quad 1 \%$

- Inkubation für 1 Stunde bei RT im Szintillationszähler 


\subsubsection{Oxidation mit ABTS-Kationradikalen}

Für Zwecke des ABTS $^{\bullet+}$ Reduktionstests (Re et al., 1999) wurden unterschiedliche Verhältnisse von Indolen zu ABTS ${ }^{\bullet+}$ getestet.

\section{Reaktionsansätze:}

Verhältnis von Melatonin zu ABTS ${ }^{\bullet+}$

Reaktionsansatz:

Endkonzentrationen:

$25 \mu \mathrm{L} \mathrm{ABTS}{ }^{\bullet+}$

(Ext. 1,44) $40 \mu \mathrm{M}$

$25 \mu \mathrm{L}$ Indol in $\mathrm{EtOH}$

$950 \mu \mathrm{L} \mathrm{H}_{2} \mathrm{O}$

* folgende Indole wurden getestet:

Tryptamin, 5-Methoxytryptamin, $N$-Acetyltryptamin, $N$-Acetylserotonin, Serotonin und Melatonin

Für die Produktanalyse aus Melatonin oder seinem Metaboliten AFMK erwies sich ein Verhältnis zum $\mathrm{ABTS}^{\bullet+}$ von 1:10 als günstig.

\section{Einfacher Ansatz:}

Verhältnis von Melatonin zu ABTS ${ }^{\bullet+}$

Reaktionsansatz:

Endkonzentrationen:

7,5 $\mathrm{mL} \mathrm{ABTS}^{\bullet+}(4,0 \mathrm{mM})$

3,0 mM

$1,0 \mathrm{~mL}$ Indol / Indolmetabolit* in EtOH $(3,0 \mathrm{mM}) \quad 0,3 \mathrm{mM}$

$1,5 \mathrm{~mL} \mathrm{H}_{2} \mathrm{O}$

* folgende Indole / Indolmetabolite wurden getestet:

Melatonin, AFMK, Produkt (2a/b): N-(1-formyl-5-methoxy-3-oxo-2,3-dihydro-1H-indol-2ylidenemethyl)-acetamid, Produkt (3): $N$-(1-formyl-2-hydroxy-5-methoxy-3-oxo-2,3-dihydro1H-indol-2-ylmethyl)-acetamid.

Bei den Indolmetaboliten (2a/b) und (3) konnten lediglich circa Konzentration angenommen werden.

Präparative Ansätze enthielten das Vielfache (z.B. 30 - 50 fache) dieses Volumens, wobei sowohl die Reaktion als auch Extraktion in $1 \mathrm{~L}$ Glasflaschen erfolgte. 


\subsection{Chromatographie}

\subsubsection{Analytische Dünnschichtchromatographie}

Zur Analyse der Produkte wurden industriell hergestellte DC-Platten (siehe Geräte) der Firma Merck verwendet.

\section{Extraktion:}

Um photokatalytische Reaktion zu vermeiden, wurden mit Alufolie umwickelte Szintillationsröhrchen für die Reaktionsansätze benutzt. Für einige photo- und fluorometrische Messungen wurden die Reaktionsansätze auch direkt in den Küvetten angesetzt.

Für die Extraktion wurde das Reaktionsgemisch jeweils in $50 \mathrm{~mL}$ Falcon-Röhrchen überführt und mit dem 2-fachen Volumen an Ethylacetat versetzt. Die Röhrchen wurden verschlossen und mit Alufolie gegen Lichteinfall umwickelt. Anschließend wurden sie auf dem Schüttler 15 min bei maximaler Geschwindigkeit in horizontaler Position geschüttelt. Nach erfolgter Phasentrennung wurde die obere organische Phase mit einer Pasteurpipette abgenommen und in Kristallisationsschälchen übertragen und verteilt. Danach wurde die organische Phase in einem lichtabgeschirmten Glasexsikkator mit Hilfe einer Wasserstrahlpumpe auf ein Volumen auf ca. 0,5 mL eingeengt. Dieses Restvolumen wurde in Eppendorf-Cups überführt und in einer Zentrifuge kurz 5 min bei 5000 rpm vom evtl. noch vorhandenen Wasser befreit. Die jetzt wasserfreie obere Phase konnte zur weiteren Verwendung dünnschichtchromatographisch aufgetrennt oder photometrisch bzw. fluorometrisch untersucht werden. 
Zur bestmöglichsten Auftrennung der einzelnen Produktbande kamen unterschiedliche Eluenten zum Einsatz.

\section{Eluenten:}

Ethylacetat / Methanol

Verhältnisse: $\quad 9,9: 0,1 ; 9,5: 0,5 ; 9: 1 ; 7: 3 ; 5: 5$

Methanol / $\mathrm{H}_{2} \mathrm{O}$

Verhältnisse: $\quad 9: 1 ; 8: 2 ; 7: 3$

Butanol / Eisessig : $\mathrm{H}_{2} \mathrm{O}$

Verhältnisse: $\quad 16: 1: 2 ; 16: 0,5: 1 ; 16: 0,25: 0,5$

Dichlormethan / Methanol

Verhältnisse: $\quad 9,8: 0,2 ; 9,5: 0,5 ; 9: 1 ; 8,5: 1,5 ; 8: 2$

Propanol / HCl

Verhältnisse: $\quad \mathrm{HCl} \mathrm{pH} \mathrm{4,5} \mathrm{(9,5} \mathrm{:} \mathrm{0,5);} \mathrm{HCl} \mathrm{pH} \mathrm{4,5} \mathrm{(9} \mathrm{:} \mathrm{1);} \mathrm{HCl} \mathrm{pH} \mathrm{3,0} \mathrm{(9} \mathrm{:} \mathrm{1);}$ $\mathrm{HCl}$ pH 2,6 (7 : 3)

Nach Beendigung des Laufes wurden die DC-Platten kurz im Dunkeln getrocknet und sofort unter einer UV-Lampe auf das Vorhandensein indolischer Produkte bei $254 \mathrm{~nm}$ oder kynurischer Produkte bei 366 nm überprüft. Die einzelnen Produktbanden wurden markiert und halbquantitativ in verschiedene Intensitätsstufen eingeteilt.

\section{$\underline{\text { Reelution }}$}

Zur weiteren Charakterisierung bzw. Aufreinigung wurde die jeweilige Bande aus der DCPlatte mit Hilfe eines Skalpells herausgeschnitten und in passender Größe in Kristallisationsschälchen verteilt. Die Reelution erfolgte auf einem Schüttler mit jeweils $3 \mathrm{~mL}$ Ethanol. Die Schälchen wurden 15 min behutsam geschüttelt und der Extrakt in ein Eppendorf-Cup überführt. Evtl. vorhandene Kieselgelreste wurden in der Zentrifuge bei 5000 rpm in 10 min aus dem Extrakt entfernt. 


\subsubsection{Präparative Dünnschichtchromatographie}

Auftrennung und Eluierung der Produkte durch PTLC

Für die Produktanalyse wurden in der präparativen Dünnschichtchromatographie eigens hergestellte PTLC-Platten (siehe Chemikalien) verwendet. Ein Vielfaches des Reaktionansatzes (2.3.3) wurde hergestellt und ebenso behandelt wie in der analytischen Dünnschichtchromatographie.

Für die bestmögliche Auftrennung der einzelnen Produkte wurde der Eluent Ethylacetat / Methanol $(9,5: 0,5)$ verwendet.

Nach erfolgter Auftrennung wurde die jeweilige Bande aus der PTLC-Platte mit Hilfe eines Skalpells herausgeschnitten, im Lösungsmittelgemisch DCM / MeOH (1:1) reeluiert und anschließend filtiert. Mit Hilfe von kleinen Fertigfolien für die Dünnschichtchromatographie (40×80 mm, 2.1.2) wurde die Reinheit der Substanz überprüft. Zur weiteren Untersuchung wurde die Substanz mit Hilfe eines Rotationsverdampfers und einer Vakuumpumpe hiernach vollständig getrocknet.

\section{Säulenchromatographie}

Zur Produkttrennung wurde eine Sephadex-Säule LH-20 (2.1, 2.2) mit dem Laufmittel DCM / MetOH (1 : 1) hergestellt, die zum Schutz der lichtempfindlichen Substanzen mit Alufolie ummantelt war. Die verschiedenen Produktfraktionen in der Säule konnten durch ihre Tageslichtfarbe (TL) oder ihrer Fluoreszenz unter UV-Licht (366 und 254 nm) voneinander unterschieden werden. Die Reinheit der Fraktionen wurde wiederum mit kleinen Fertigfolien für die Dünnschichtchromatographie $(40 \times 80 \mathrm{~mm}, 2.1 .2)$ überprüft. Die Fraktion mit der gesuchten Substanz wurde mit Hilfe eines Rotationsverdampfers und einer Vakuumpumpe hiernach vollständig getrocknet.

\subsection{Chemilumineszenzmessung:}

Die Oxidation von 6-Methoxymelatonin wurde anhand der Messung der Chemilumineszenz mit Hilfe eines auf $20^{\circ} \mathrm{C}$ temperierten Szintillationszählers ( Packard, Modell 3330) verfolgt. Im Verlauf der Oxidation von Indolen mit freien Radikalen kann ein kurzlebiges DioxetanZwischenprodukt gebildet werden, bei dessen Zerfall ein Photon emittiert wird. Wegen der Erfassung der Photonenemission mit einem Szintillationsspektrometer wurden die Meßdaten 
als cpm ausgegeben. Der Hintergrund bei diesem Versuch betrug $17.000 \mathrm{cpm}$ und wurde von den Daten abgezogen.

Einstellung des Szintillationszählers:

Preset time: $\quad 1 \mathrm{~min}$

Preset counts: $\quad 900.000$

Gain: $\quad 70 \%$

Window: $\quad 40-100$

Coincidence: off

Die Auswertung erfolgte nur über einen der drei Kanäle, bei den anderen war die Verstärkung sehr niedrig eingestellt, um die Zeitvorwahl (preset time) auf einen Kanal zu beschränken. Es wurde im Betriebsmodus „repeat“ gemessen.

Über einen an den Szintillationszähler gekoppelten Prozeßrechner wurden die Daten aufgenommen und über eine Personalcomputer abgerufen. Die Daten wurden als Mediane von 10 min Intervallen ausgegeben.

\subsection{Fluorometrie}

Zur näheren Charakterisierung einer Substanz wurden von dieser Excitations- und Emissionspektren in Makroquarzküvetten für Fluorometrie bei $25^{\circ} \mathrm{C}$ aufgenommen.

Einstellungen am Spektralfluorometer

Spaltgröße: $\quad 4 \mathrm{~mm}$

Messmodus: Ratio

Spalt am Photomultiplier: $\quad 0,5-1,0$

Sensitivität: $\quad \quad 0,1-100$

Response: $\quad 3$

Chopper: $\quad$ On 


\section{$2.7 \quad$ Photometrie}

Weitere Untersuchungen von Substanzen erfolgten in einem Zeiss-Spektralphotometer, das über einen Durchflußthermostaten auf $25^{\circ} \mathrm{C}$ konstant gehalten wurde. Hierfür wurden entsprechende Plastik- und Glasküvetten verwendet. Leerwertmessungen wurden mit den jeweiligen Lösungsmitteln, Puffern oder aq. dest. durchgeführt.

\subsection{Massenspektroskopie}

Die Aufnahme der Massenspektren erfolgte in der von Dr. H. Frauendorf geleiteten Abteilung Massenspektrometrie (Institut für Organische und Biomolekulare Chemie, Georg-AugustUniversität Göttingen)

\section{$2.9 \quad$ HPLC-MS}

Zur Analyse eines Reaktionsgemisches wurde eine Hochdruckflüssigkeitschromatographie mit anschließender Massenspektroskopie (HPLC-MS) ebenfalls in der von Dr. H. Frauendorf geleiteten Abteilung Massenspektrometrie durchgeführt.

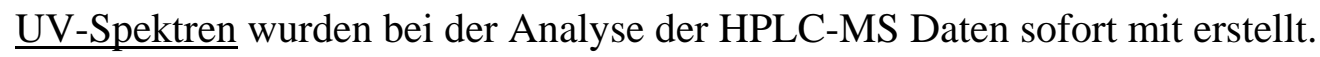

\section{$\underline{2.10 ~ N M R}$}

Die Messung der NMR-Spektren wurde von der Arbeitsgruppe Kernresonanz (Institut für Organische und Biomolekulare Chemie, Georg-August-Universität Göttingen) unter der Leitung von Herrn Dipl.-Chem. R. Machinek durchgeführt:

Kopplungskonstanten $\left(J\right.$ ) bei den ${ }^{1} \mathrm{H}-\mathrm{NMR}-$ Spektren werden in $\mathrm{Hz}$ angegeben. Abkürzungen: $\mathrm{s}=$ Singulett, $\mathrm{d}=$ Dublett, $\mathrm{dd}=$ Doppeldublett, $\mathrm{t}=$ Triplett, $\mathrm{q}=$ Quartett, $\mathrm{br}=$ breit .

Die chemische Verschiebung wird in $\delta$-Werten relativ zu Tetramethylsilan als internem Standard angegeben. 


\subsection{DBE-Wert}

$$
\mathrm{DBE}=\text { Anzahl der C-Atome }-\frac{\text { Anzahl der H-Atome }}{2}+\frac{\text { Anzahl der N-Atome }}{2}+1
$$

Bei der Charakterisierung neuer Substanzen kann der DBE-Wert (DBE = Double Bond Equivalence) Hilfestellung leisten, indem er die Anzahl der Doppelbindungen in der Substanz angibt. 


\section{Ergebnisse}

\subsection{Photokatalytischer Umsatz von Indolen mit Protoporphyrin-IX}

\subsubsection{Umsatz von Melatonin mit Protoporphyrin-IX}

Unter dem Einfluss des Photokatalysators Protoporphyrin-IX wird Melatonin in erheblichen Maße umgesetzt. Es erscheint ein Produkt mit der charakteristischen Fluoreszenz von AFMK (Abb. 6). Dies bestätigt sich in der dünnschichtchromatographischen Analyse (Abb. 5, Tab. 2). Als einziges Oxidationsprodukt ist AFMK mit dem für ihn typischen RF-Wert nachzuweisen.

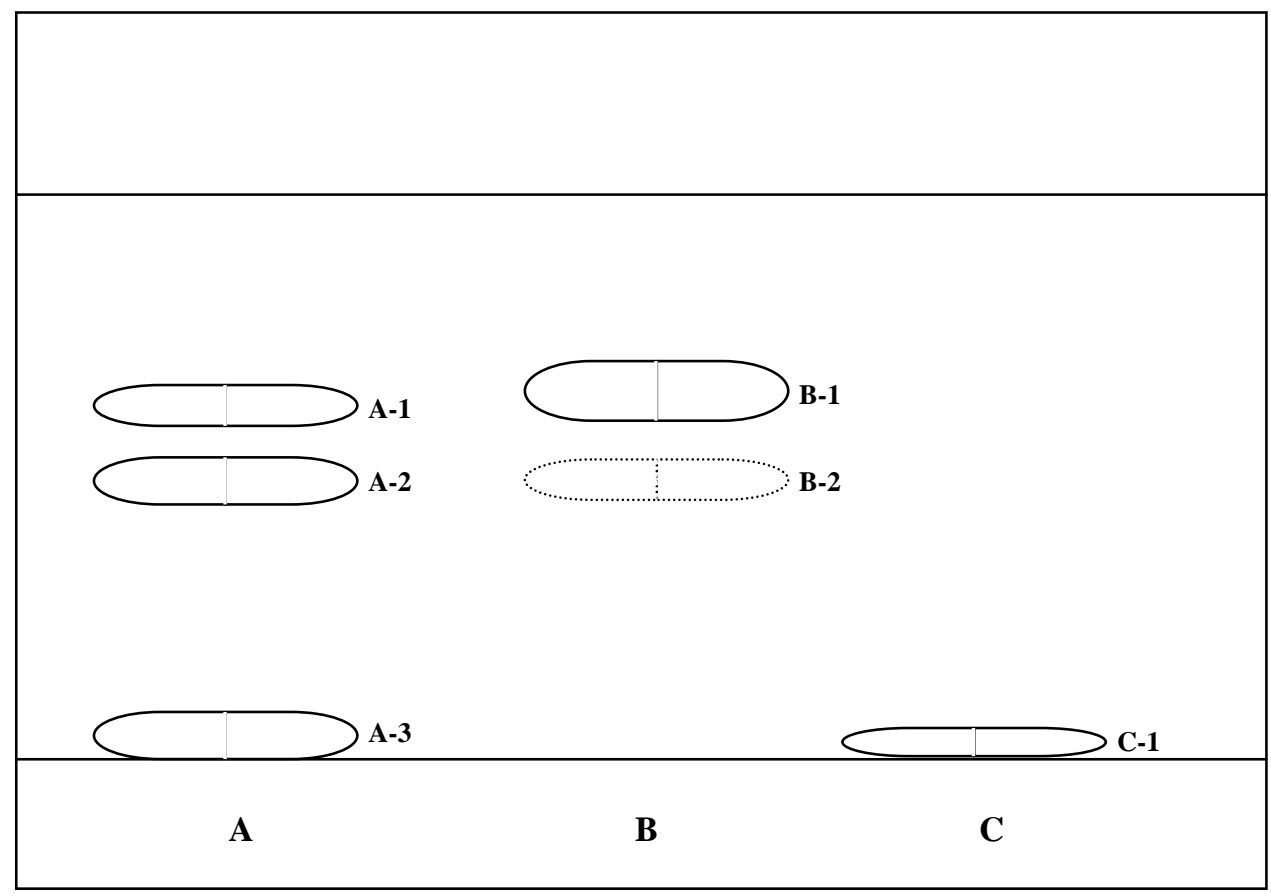

Abb. 5: Chromatogramm von Melatonin und dessen Oxidationsprodukte. Laufmittel: Ethylacetat / MetOH (9:1); Laufstrecke $11,2 \mathrm{~cm}$; Spur A: Melatonin $4 \mathrm{mM}$, P-IX $40 \mu \mathrm{M} 3$ Std. belichtet (Konzentration bei Reaktionsstart); Spur B: Melatonin Standard; Spur C: P-IX unbelichtet.

Tab. 2: Charakterisierung der Banden aus Abb. 10

\begin{tabular}{|c|l|c|l|c|l|c|}
\hline Bande & \multicolumn{1}{|c|}{ Spur A } & $\begin{array}{c}\text { Rf- } \\
\text { Wert }\end{array}$ & \multicolumn{1}{|c|}{ Spur B } & $\begin{array}{c}\text { Rf- } \\
\text { Wert }\end{array}$ & Spur C & $\begin{array}{c}\text { Rf- } \\
\text { Wert }\end{array}$ \\
\hline 1 & Melatonin & 0,63 & Melatonin & 0,64 & P-IX & 0,03 \\
\hline 2 & AFMK & 0,47 & AFMK & 0,47 & & \\
\hline 3 & P-IX & 0,02 & & & & \\
\hline
\end{tabular}


Abb. 6: Fluoreszenz von Melatonin und dessen Oxidationsprodukte. Melatonin $4 \mathrm{mM}, \quad$ P-IX $\quad 40 \mu \mathrm{M}$ (Konzentration bei Reaktionsstart); linke Ordinate: - - Melatonin den Zeitpunkten 0 Std. und $-\mathbf{-} 3$ Std.; rechte Ordinate: $-\bigcirc$ Oxidationsprodukt den Zeitpunkten 0 Std. und $\triangle 3$ Std. Die Eduktmessung erfolgte bei einer Excitation von $285 \mathrm{~nm}$. Die Produktmessung bei einer Excitation von $340 \mathrm{~nm}$.

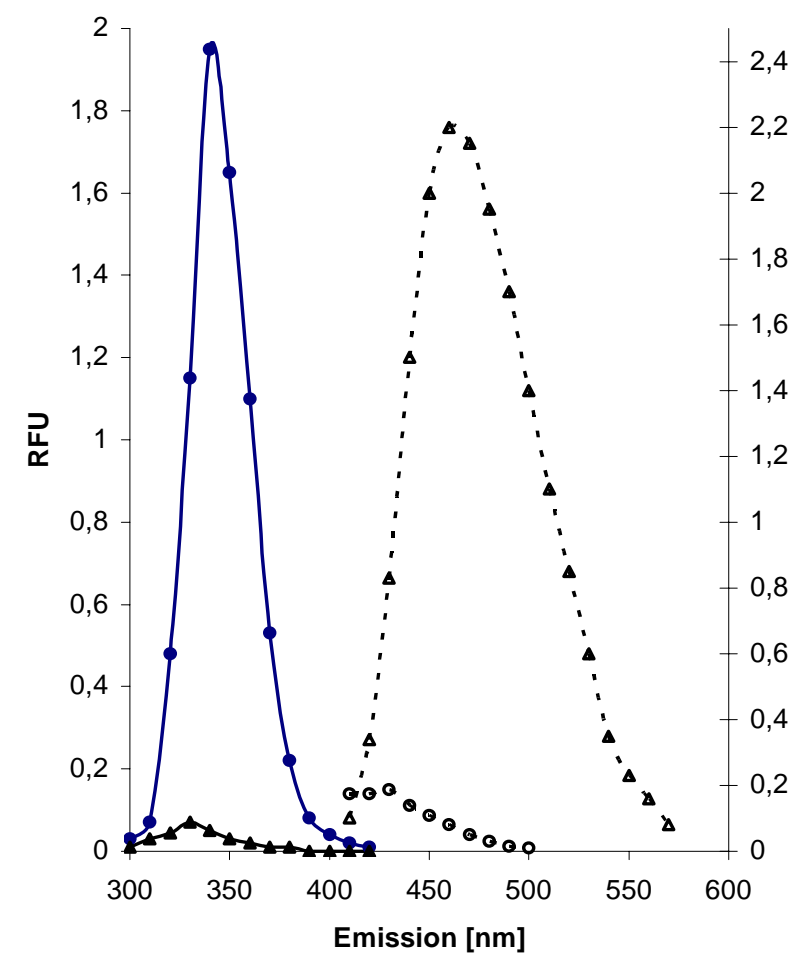

Da die im Experiment von Abb. 10 verwendeten Konzentrationen für eine quantative Fluorometrie zu hoch sind, wie die Eichkurve (Abb. 9) ausweist, wurden entsprechende Experimente mit gleichen Eduktkonzentrationen, aber nachträglicher Verdünnung auf 1/50 für die Messung durchgeführt. Hierbei zeigt sich, dass im Reaktionszeitraum nahezu die Hälfte des Melatonins umgesetzt wurde (Abb. 12)

Abb. 7: Zeitliche Verfolgung des Umsatzes von Melatonin durch Fluorometrie. Melatonin $4 \mathrm{mM}$, Protoporphyrin-IX $40 \quad \mu \mathrm{M}$ (Endkonzentration).

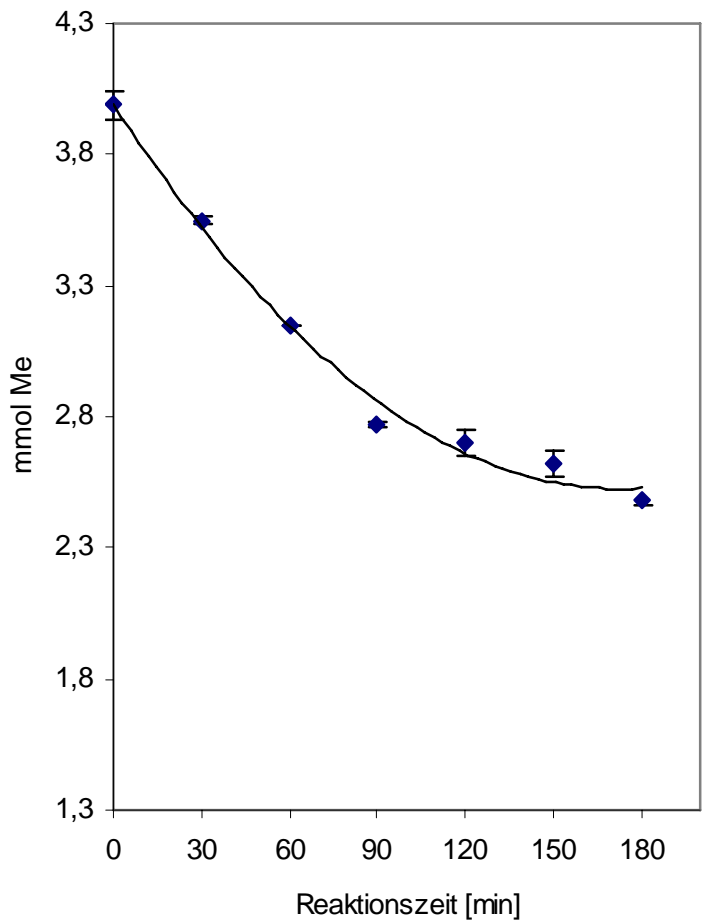

Seite 24 


\subsubsection{Umsatz von 5-Methoxytryptamin mit Protoporphyrin-IX}

Überraschenderweise führte der photokatalytische Umsatz des Strukturanalogs 5-Methoxytryptamin durch Protoporphyrin-IX bei gleichen Ausgangskonzentrationen zu einer großen Zahl unterschiedlicher Produkte (Abb. 8; Tab. 3). Der zeitliche Verlauf des Umsatzes ließ sich nach der Verdünnung auf 1/50 näherungsweise bestimmen (Abb. 9). Auch hier wird etwa die Hälfte des Indolamins im Reaktionszeitraum photokatalytisch oxidiert.

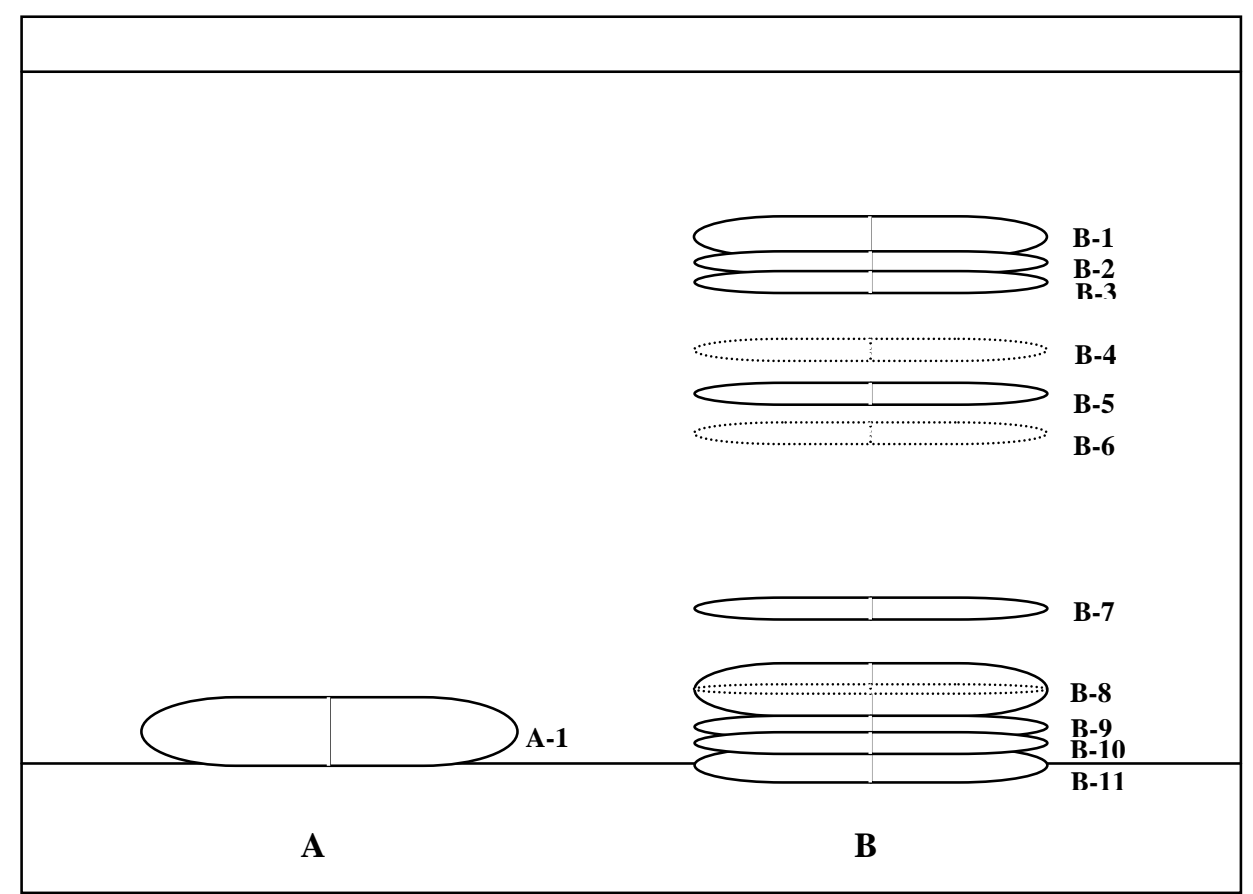

Abb. 8: Chromatogramm von 5-Methoxytryptamin und dessen Oxidationsprodukten. Laufmittel: Ethylacetat / MetOH (7:3); Laufstrecke 15,5 cm; Spur A: 5-MT-Standard, Spur B: 5-MT $4 \mathrm{mM}$, P-IX $40 \mu \mathrm{M}$ belichtet 3 Std. (Konzentrationen bei Reaktionsstart)

Tab. 3: Charakterisierung der Banden aus Abb. 8

\begin{tabular}{|c|l|c|l|c|}
\hline Bande & \multicolumn{1}{|c|}{ Spur A } & $\begin{array}{c}\text { Rf- } \\
\text { Wert }\end{array}$ & \multicolumn{1}{|c|}{ Spur B } & Rf-Wert \\
\hline 1 & $254++5-\mathrm{MT}$ & 0,00 & $366++/ 254+$ & 0,78 \\
\hline 2 & & & 366 rötlich & 0,73 \\
\hline 3 & & & $366++/ 254+$ & 0,70 \\
\hline 4 & & & $366+$ & 0,60 \\
\hline 5 & & & $254++$ & 0,54 \\
\hline 6 & & & $366+$ & 0,48 \\
\hline 7 & & & $254+$ & 0,22 \\
\hline 8 & & & $254+$ & 0,12 \\
\hline 9 & & & $254++$ & 0,12 \\
\hline 10 & & & $254+$ & 0,05 \\
\hline 11 & & & $254+5-\mathrm{MT}$ & 0,03 \\
\hline
\end{tabular}




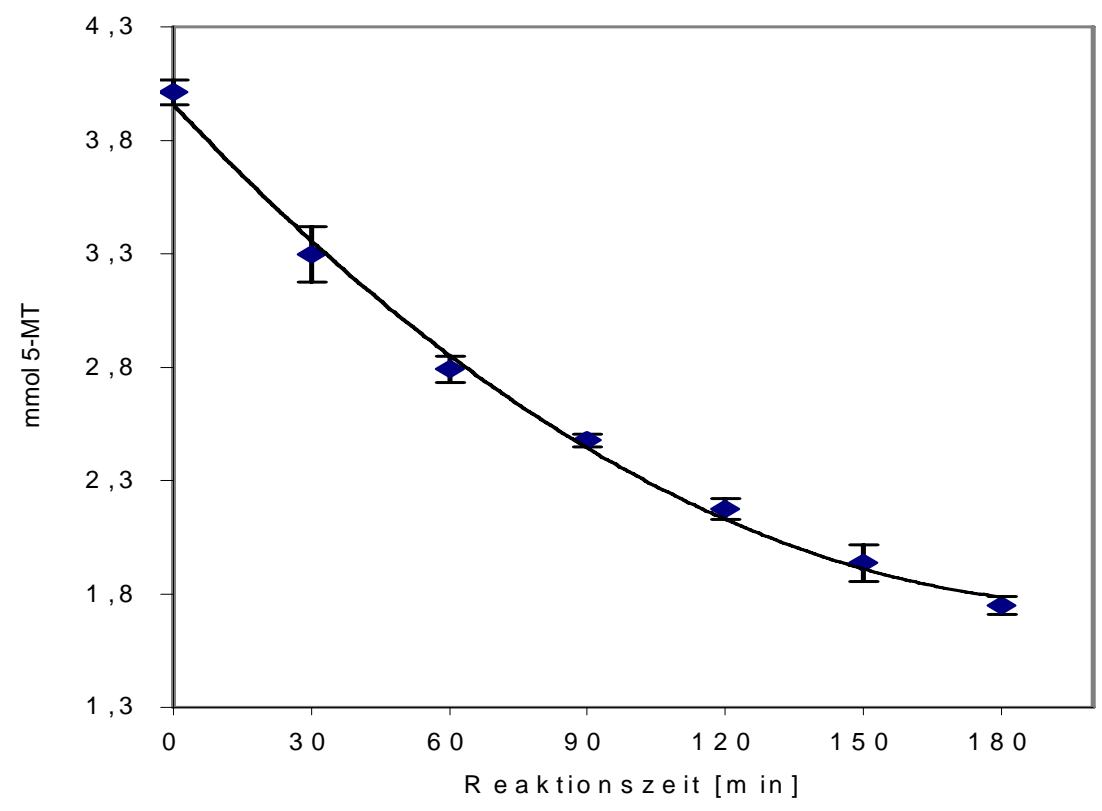

Abb. 9: Zeitliche Verfolgung des Umsatzes von 5-Methoxytryptamin durch Fluorometrie. 5-Methoxytryptamin $4 \mathrm{mM}$, Protoporphyrin-IX $40 \mu \mathrm{M}$ (Endkonzentration). 


\subsubsection{Umsatz von $N$-Acetyltryptamin mit Protoporphyrin-IX}

Die photokatalytische Reaktion von $N$-Acetyltryptamin zeigt ein ähnliches Verhalten wie Melatonin (Abb. 10; Tab. 4). Der Umsatz des Edukts führt hauptsächlich zu einem Produkt. Die fluorometrische Analyse (Abb. 11) zeigt, daß $N$-Acetyltryptamin zu einem großen Teil umgesetzt wurde. Der zeitliche Verlauf (Abb. 12) zeigt nach einer Verdünnung von 1:60 einen Umsatz von weniger als der Hälfte.

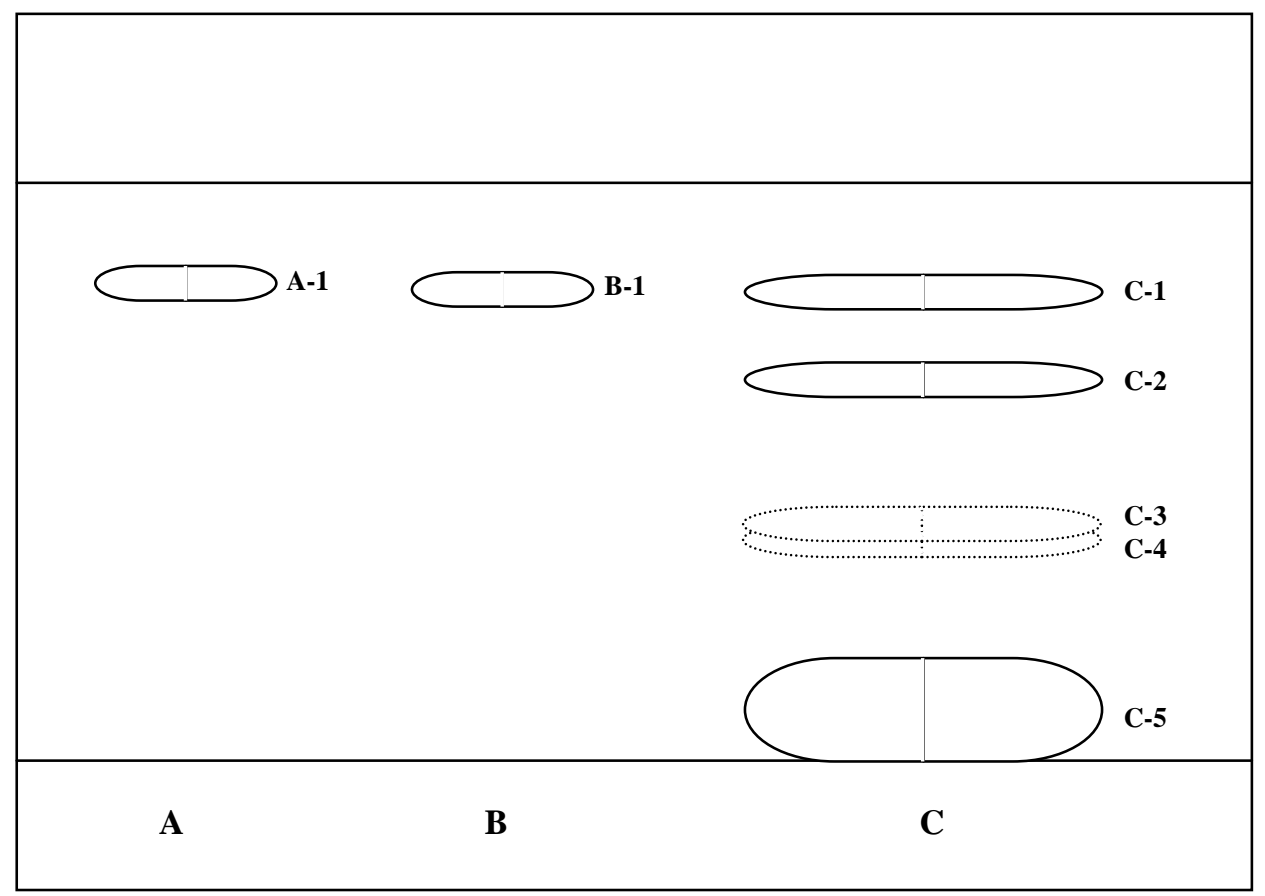

Abb. 10: Chromatogramm von $N$-Acetyltryptamin und dessen Oxidationsprodukten. Laufmittel: Ethylacetat / MetOH (7:3); Laufstrecke 12,9 cm; Spur A: NAT unbelichtet; Spur B: NAT belichtet 3 Std.; Spur C: P-IX $40 \mu \mathrm{M}$, NAT $4 \mathrm{mM}$ belichtet 3 Std. (Endkonzentration)

Tab. 4: Charakterisierung der Banden aus Abb. 10

\begin{tabular}{|c|l|l|l|l|l|c|}
\hline Bande & \multicolumn{1}{|c|}{ Spur A } & $\begin{array}{c}\text { Rf- } \\
\text { Wert }\end{array}$ & \multicolumn{1}{|c|}{ Spur B } & $\begin{array}{c}\text { Rf- } \\
\text { Wert }\end{array}$ & \multicolumn{1}{|c|}{ Spur C } & $\begin{array}{c}\text { Rf- } \\
\text { Wert }\end{array}$ \\
\hline 1 & NAT & 0,85 & NAT & 0,84 & NAT & 0,83 \\
\hline 2 & & & & & $256+++/ 366+$ & 0,68 \\
\hline 3 & & & & & $366(+)$ & 0,44 \\
\hline 4 & & & & & $254(+)$ & 0,38 \\
\hline 5 & & & & & P-IX & 0,00 \\
\hline 6 & & & & & & \\
\hline
\end{tabular}


Abb. 11: Fluoreszenzmessung von $\mathrm{N}$-Acetyltryptamin und dessen Oxidationsprodukten. NAT $4 \mathrm{mM}$, P-IX $\quad 40 \mu \mathrm{M} \quad$ (Konzentrationen bei Reaktionsstart); Linke Ordinate: $\mathrm{N}$-Acetyltryptamin zu den Zeitpunkten 0 Std. und -3 Std.; rechte Ordinate: $-\odot$ Oxidationsprodukte $\mathrm{zu}$ den Zeitpunkten 0 Std. und $\triangle 3$ Std. Die Eduktmessung erfolgte bei einer Excitation von $280 \mathrm{~nm}$. Die Produktmessung erfolgte bei einer Excitation von $320 \mathrm{~nm}$.

Abb. 12: Zeitliche Verfolgung des Umsatzes von $N$-Acetyltryptamin durch Fluorometrie. NAT $4 \mathrm{mM}$, PIX $40 \mu \mathrm{M}$ (Endkonzentration). Die Konvertierung in Konzentration pro Zeiteinheit erfolgte mit Hilfe einer zuvor erstellten Eichkurve (Abb. 4a).
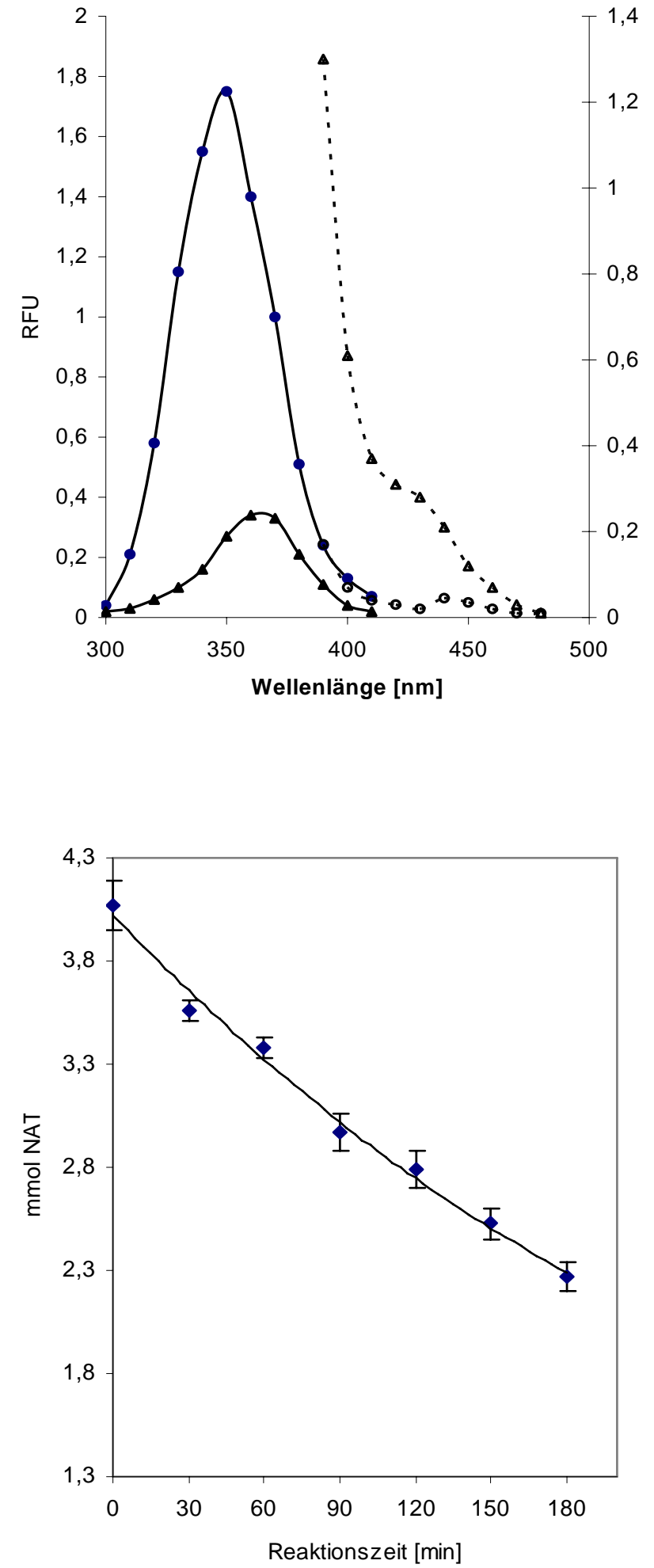


\subsubsection{Umsatz von von $N$-Acetylserotonin mit Protoporphyrin-IX}

Auch $N$-Acetylserotonin geht größtenteils in ein Produkt über (Abb. 13). Die Belichtung von $N$-Acetylserotonin alleine führt schon zur Produktbildung, wobei das Hauptprodukt, das bei der Reaktion mit Protoporphyrin-IX entsteht, auch schon zuerkennen ist. Die Fluorometrie (Abb. 14) weist eine fast vollständige Oxidation von NAS auf, was im zeitlichen Verlauf (Abb. 15) mit einem photokatalytischen Verbrauch von ca. $75 \%$ dokumentiert wird.

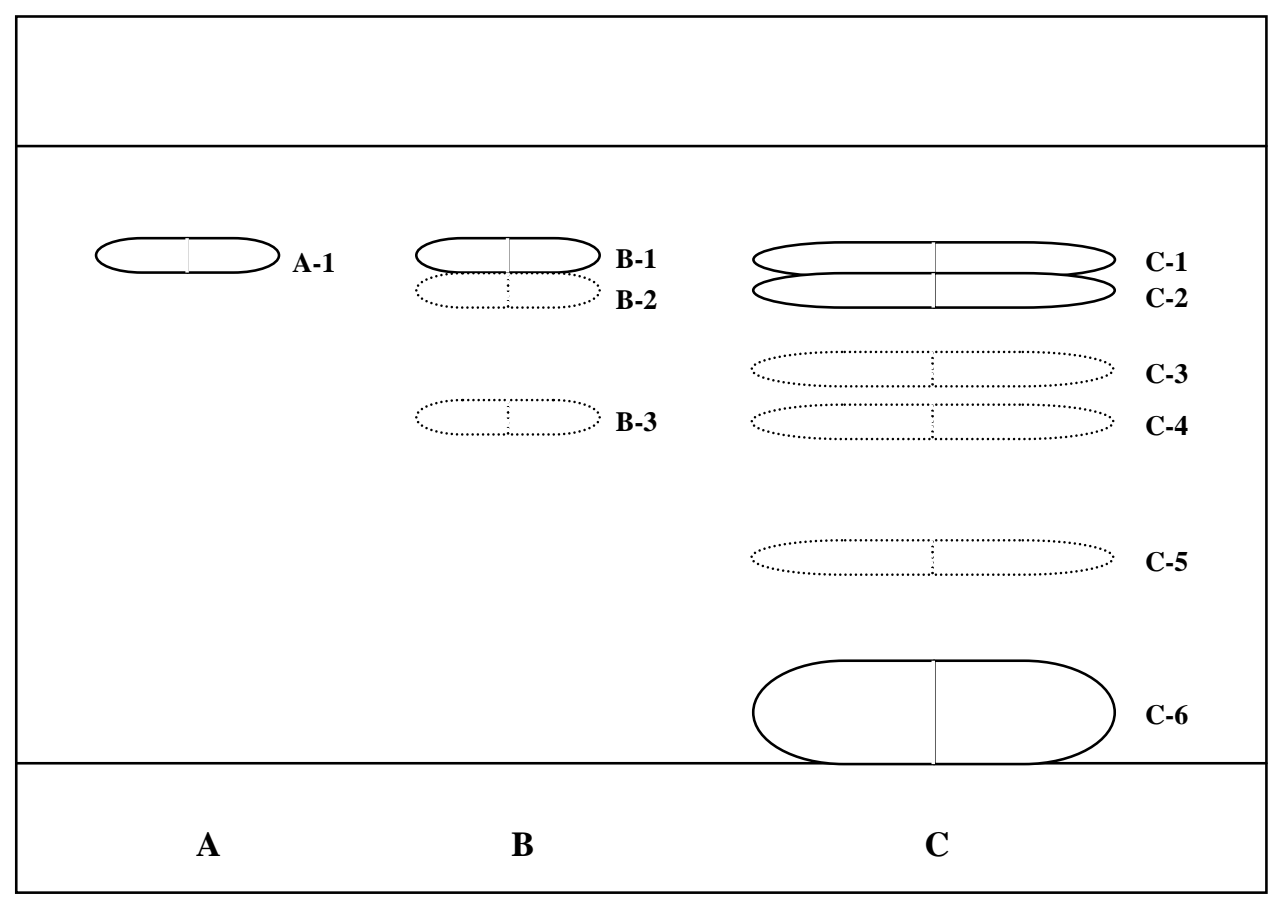

Abb. 13: Dünnschichtchromatograhie von $N$-Acetylserotonin und dessen Oxidationsprodukten in einem ethanolischen, ProtoporphyrinIX-katalysierten System. Laufmittel: Ethylacetat / MetOH (7:3); Laufstrecke 13,8 cm; Spur A: NAS unbelichtet; Spur B: NAS belichtet 3

Std.; Spur C: P-IX 40 $\mu$ M, NAS 4 mM belichtet 3 Std. (Endkonzentration)

Tab. 5: Charakterisierung der Banden aus Abb. 13

\begin{tabular}{|c|l|c|l|c|l|c|}
\hline Bande & \multicolumn{1}{|c|}{ Spur A } & $\begin{array}{c}\text { Rf- } \\
\text { Wert }\end{array}$ & \multicolumn{1}{|c|}{ Spur B } & $\begin{array}{c}\text { Rf- } \\
\text { Wert }\end{array}$ & \multicolumn{1}{c|}{ Spur C } & $\begin{array}{c}\text { Rf- } \\
\text { Wert }\end{array}$ \\
\hline 1 & NAS & 0,84 & NAS & 0,84 & NAS & 0,84 \\
\hline 2 & & & $366(+)$ & 0,78 & $366+++, 254+$ & 0,78 \\
\hline 3 & & & $254(+)$ & 0,57 & $254(+)$ & 0,65 \\
\hline 4 & & & & & $254+$ & 0,57 \\
\hline 5 & & & & & $254(+)$ & 0,34 \\
\hline 6 & & & & & P-IX & 0,00 \\
\hline
\end{tabular}


Abb. 14: Fluoreszenzmessung von $N$ Acetylserotonin und dessen Oxidationsprodukten. NAS 4 mM, P-IX $40 \mu \mathrm{M} \quad$ (Endkonzentration); Linke Ordinate: - $-\mathrm{N}$-Acetylserotonin zum Zeitpunkt 0 Std. und - zum Zeitpunkt 3 Std.; Rechte Ordinate: Oxidationsprodukt zum Zeitpunkt 0 Std. und $\triangle$ zum Zeitpunkt 3 Std. Die Eduktmessung erfolgte bei einer Excitation von $290 \mathrm{~nm}$. Die Produktmessung erfolgte bei einer Excitation von $330 \mathrm{~nm}$.

Abb. 15: Zeitliche Verfolgung des Umsatzes von $N$-Acetylserotonin durch Fluorometrie. $N$-Acetylserotonin $4 \mathrm{mM}$, Protoporphyrin-IX $40 \mu \mathrm{M}$ (Endkonzentration). Die Konvertierung in Konzentration pro Zeiteinheit erfolgte mit Hilfe einer zuvor erstellten Eichkurve (Abb. 4a).
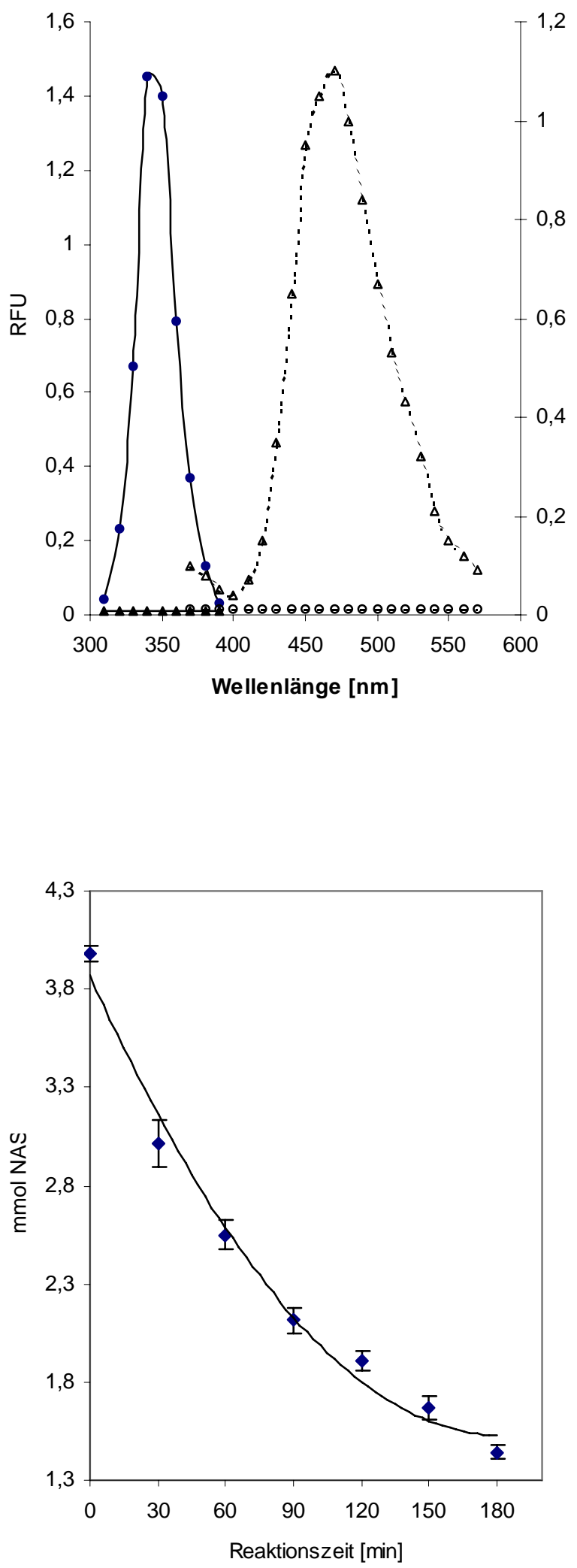


\subsubsection{Umsatz von Serotonin mit Protoporphyrin-IX}

Auch bei Serotonin kommt es durch Belichtung bereits zur Produktbildung (Abb. 16, Tab. 6). Im photokatalytischen Umsatz wird die Produktbildung weiter forciert, wobei ein zusätzliches Produkt entsteht. Die fluorometrische Analyse (Abb. 17) zeigt einen weitestgehend vollständigen Umsatz von Serotonin an, welches sich in der Bildung des Produktes, das bei $E x=290 \mathrm{~nm}$ und ein $\mathrm{Em}=340 \mathrm{~nm}$ verfolgt wurde, niederschlägt. Serotonin wurde in dieser Zeit zur Hälfte umgesetzt (Abb. 18).

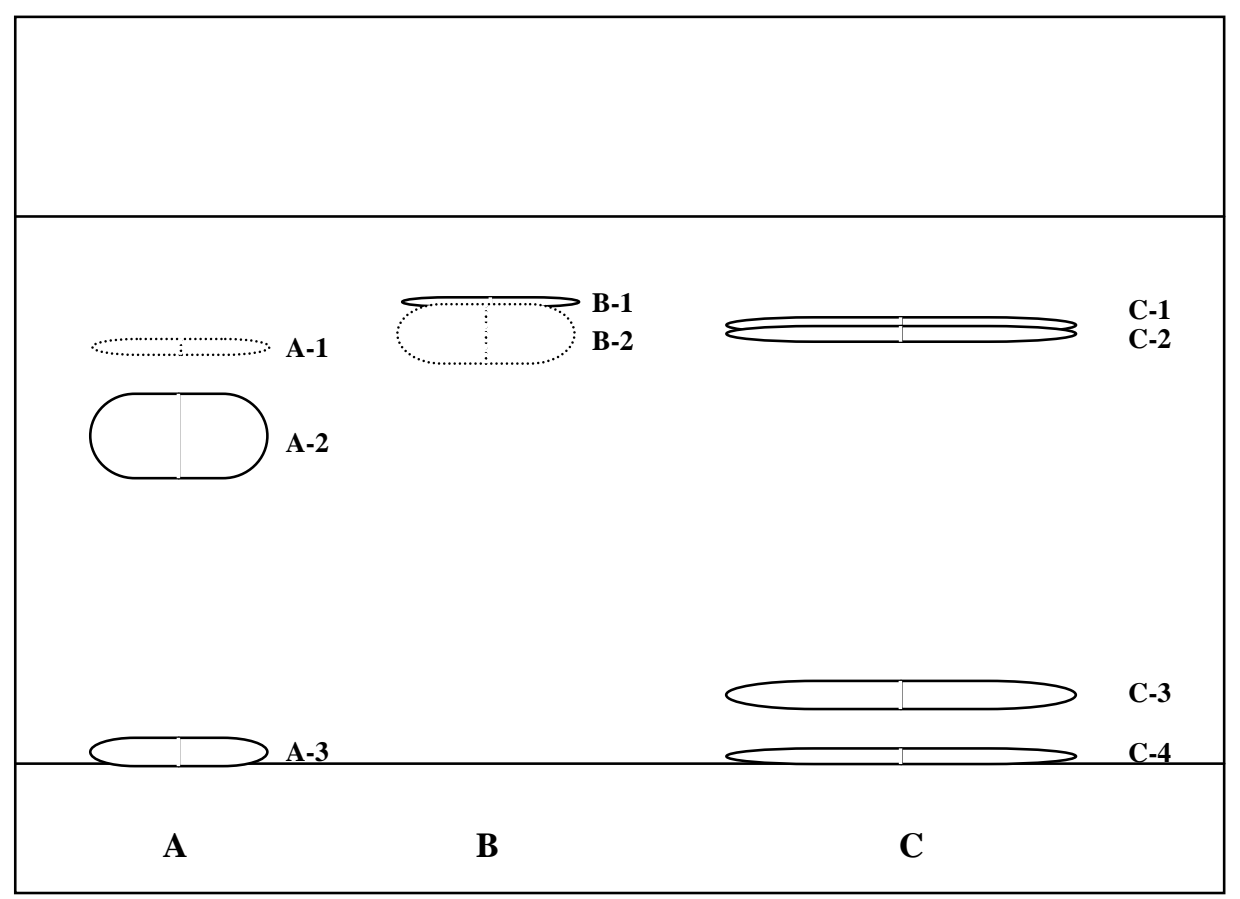

Abb. 16: Chromatogramm von Serotonin und dessen Oxidationsprodukten. Laufmittel: $\mathrm{MetOH} / \mathrm{H}_{2} \mathrm{O}$ (8:2); Laufstrecke $12,2 \mathrm{~cm}$; Spur A: Serotonin unbelichtet; Spur B: Protoporphyrin Std.; Spur C: P-IX $40 \mu \mathrm{M}$, Serotonin $4 \mathrm{mM}$ (belichtet 3 Std.). (Endkonzentration)

Tab. 6: Charakterisierung der Banden aus Abb. 16

\begin{tabular}{|c|l|c|l|c|l|c|}
\hline Bande & \multicolumn{1}{|c|}{ Spur A } & $\begin{array}{c}\text { Rf- } \\
\text { Wert }\end{array}$ & \multicolumn{1}{|c|}{ Spur B } & $\begin{array}{c}\text { Rf- } \\
\text { Wert }\end{array}$ & \multicolumn{1}{c|}{ Spur C } & $\begin{array}{c}\text { Rf- } \\
\text { Wert }\end{array}$ \\
\hline 1 & $366+$ & 0,76 & $366+, 254(+)$ & 0,84 & 366 braun, 254 (+) & 0,80 \\
\hline 2 & $254++$ & 0,59 & 366 rötlich & 0,78 & $366+$ & 0,78 \\
\hline 3 & $254+$ & 0,02 & & & $366++++$ & 0,13 \\
\hline 4 & & & & & $256(+)$ & 0,02 \\
\hline 5 & & & & & & \\
\hline
\end{tabular}


Abb. 17: Fluoreszenzmessung von Serotonin und dessen Oxidationsprodukten. Serotonin $4 \mathrm{mM}$, P-IX $40 \mu \mathrm{M}$ (Endkonzentration); Linke Ordinate: - - Serotonin zum Zeitpunkt 0 Std. und - zum Zeitpunkt 3 Std.; Rechte Ordinate: Oxidationsprodukte zum Zeitpunkt 0 Std. und $\triangle$ zum Zeitpunkt 3 Std. Die Eduktmessung erfolgte bei einer Excitation von 290 nm. Die Produktmessung erfolgte bei einer Excitation von $340 \mathrm{~nm}$.

Abb. 18: Zeitliche Verfolgung des Umsatzes von Serotonin durch Fluorometrie. Serotonin $4 \mathrm{mM}$, P-IX $40 \mu \mathrm{M} \quad$ (Endkonzentrationen). Die Konvertierung in Konzentration pro Zeiteinheit erfolgte mit Hilfe einer zuvor erstellten Eichkurve (Abb. 4b).
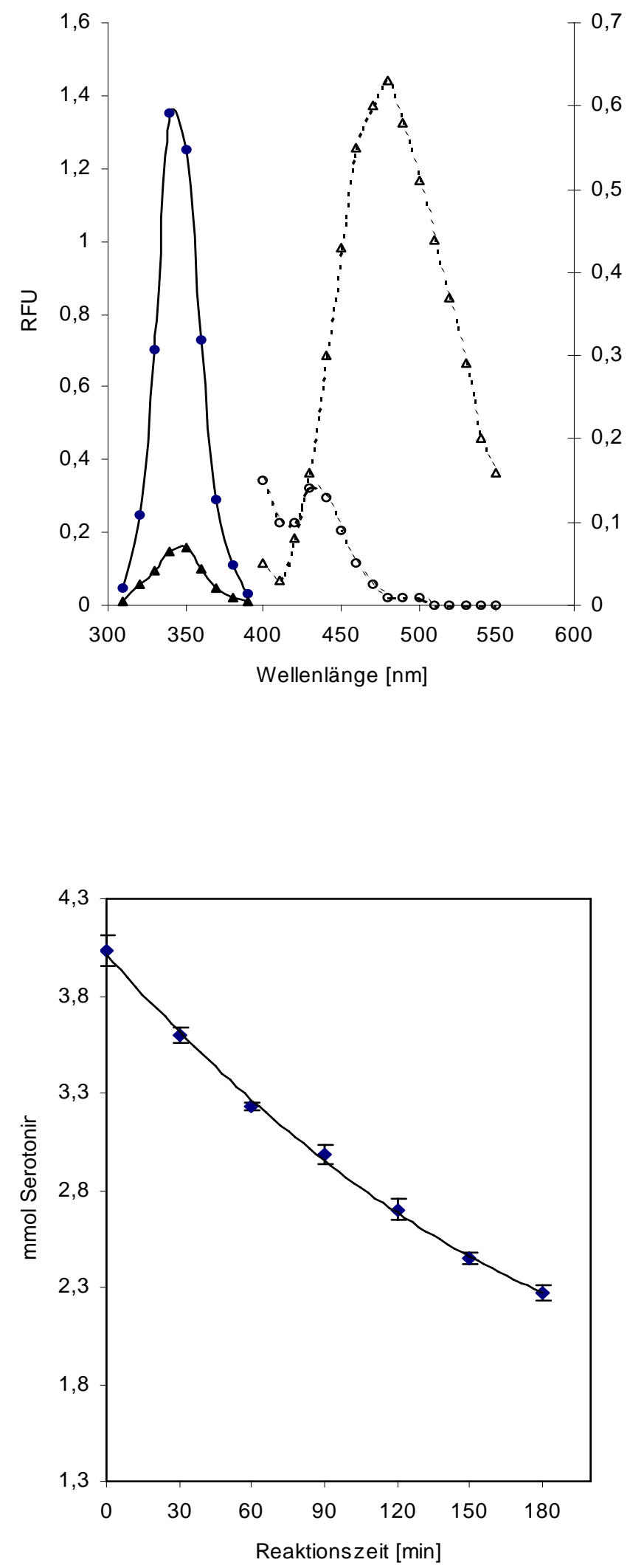


\subsubsection{Umsatz von Tryptamin mit Protoporphyrin-IX}

Das Strukturanalogon Tryptamin zeigt eine große Zahl unterschiedlichster Produkte (Abb. 19; Tab. 7). Der Umsatz des Edukts (Abb. 20) ließ sich nach der Verdünnung auf 1/60 näherungsweise bestimmen.

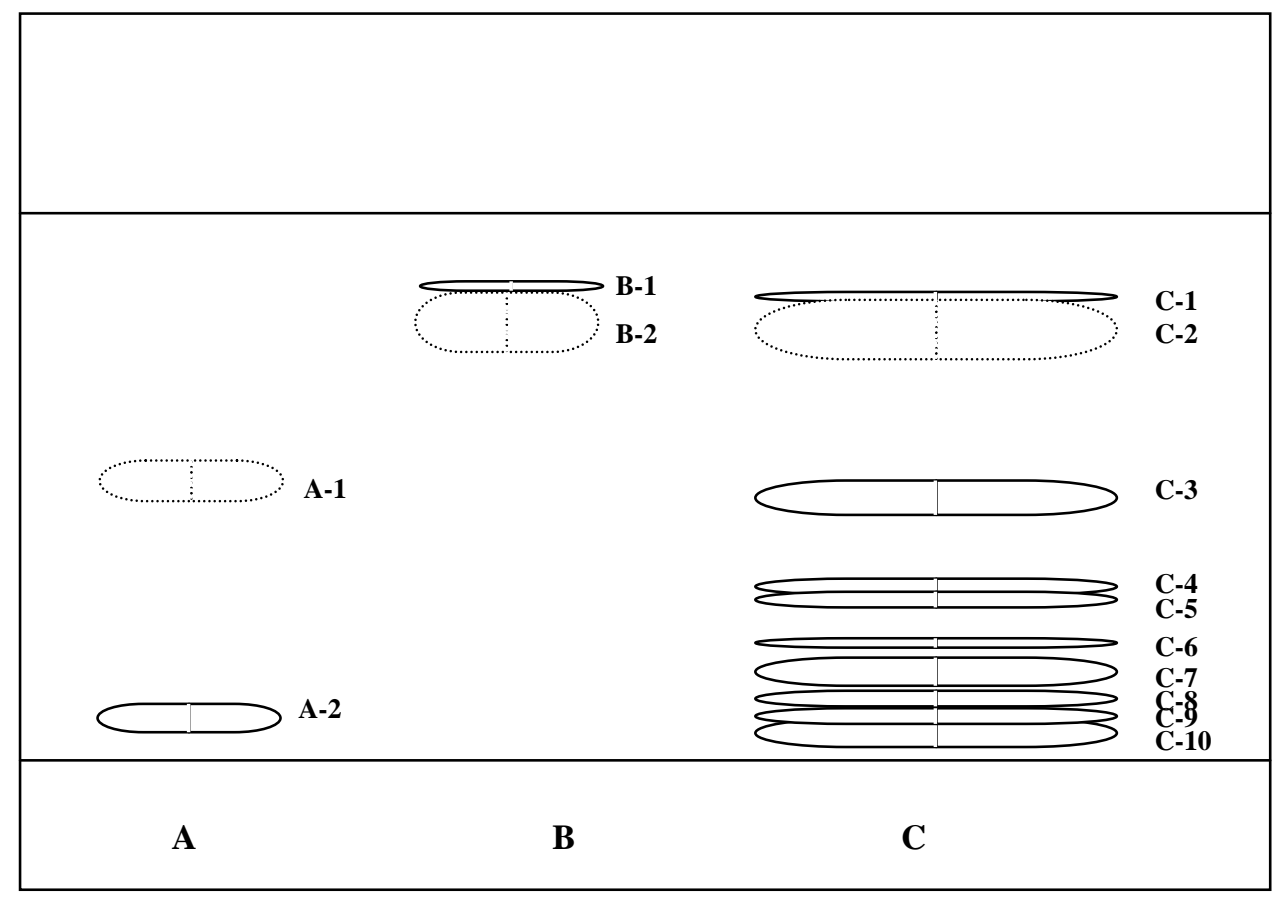

Abb. 19: Chromatogramm von Tryptamin und dessen Oxidationsprodukten. Laufmittel: Ethylacetat / MetOH (7:3); Laufstrecke 12,9 cm; Spur A: Tryptamin unbelichtet; $\underline{\text { Spur B: Tryptamin } 4}$ $\mathrm{mM}$, belichtet 3 Std.; Spur C: P-IX $40 \mu \mathrm{M}$, Tryptamin $4 \mathrm{mM}$ belichtet (3 Std.). (Endkonzentrationen).

Tab. 7: Charakterisierung der Banden aus Abb. 19

\begin{tabular}{|c|l|l|l|c|l|c|}
\hline Bande & \multicolumn{1}{|c|}{ Spur A } & & \multicolumn{1}{c|}{ Spur B } & $\begin{array}{c}\text { Rf- } \\
\text { Wert }\end{array}$ & \multicolumn{1}{c|}{ Spur C } & $\begin{array}{c}\text { Rf- } \\
\text { Wert }\end{array}$ \\
\hline 1 & $254+$ & 0,50 & $366+++$ P-IX & 0,86 & $366+$ & 0,84 \\
\hline 2 & Tryptamin & 0,04 & $366(+)$ & 0,79 & $366(+)$ & 0,78 \\
\hline 3 & & & & & $254+$ & 0,46 \\
\hline 4 & & & & & $254(+)$ & 0,30 \\
\hline 5 & & & & & $254(+)$ & 0,27 \\
\hline 6 & & & & & $366(+)$ & 0,19 \\
\hline 7 & & & & & $254+$ & 0,13 \\
\hline 8 & & & & & $366+++$ & 0,09 \\
\hline 9 & & & & & $254+$ & 0,05 \\
\hline 10 & & & & & $366(+)$ & 0,02 \\
\hline
\end{tabular}


Abb. 20: Zeitliche Verfolgung des Umsatzes von Tryptamin durch Fluorometrie.

Tryptamin $4 \quad \mathrm{mM}, \quad$ P-IX $\quad 40 \quad \mu \mathrm{M}$ (Endkonzentration). Die Konvertierung in Konzentration pro Zeiteinheit erfolgte mit Hilfe einer zuvor erstellten Eichkurve (Abb.4b).

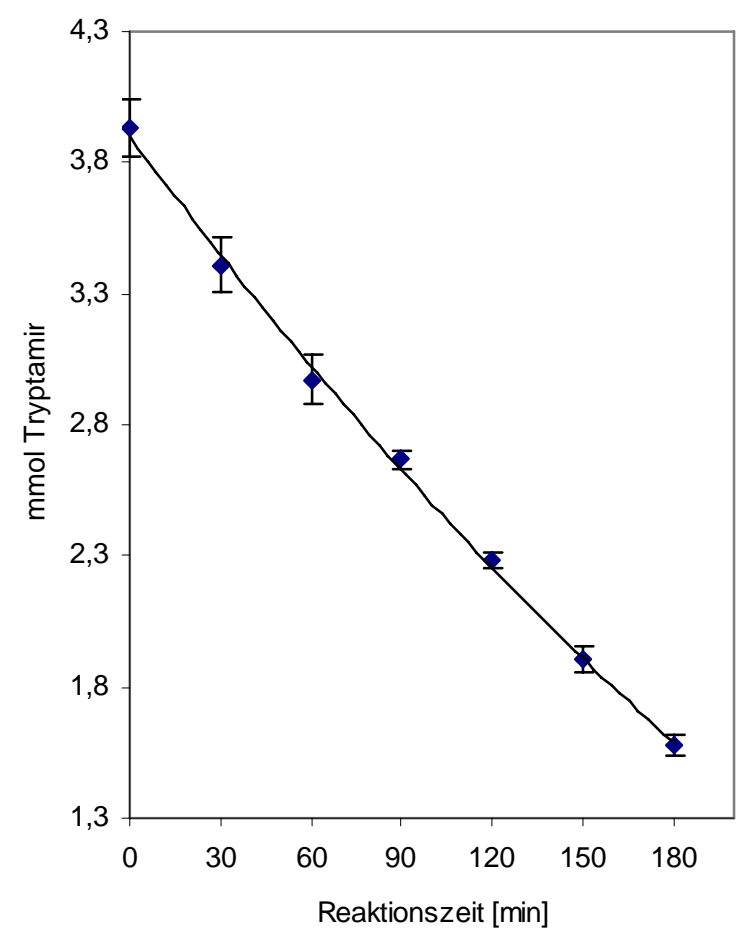




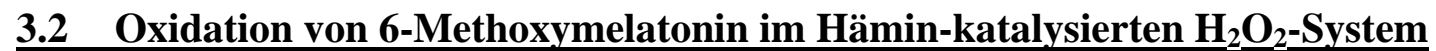

6-Methoxymelatonin wird im Hämin-katalysierten $\mathrm{H}_{2} \mathrm{O}_{2}$-System im Gegensatz zu Melatonin sehr rasch oxidiert (Abb. 21, 22).

Dies zeigt sich eindeutig in der dünnschichtchromatographischen Analyse (Abb. 23, 24; Tab. 8, 9). Die Lumineszenzreaktion ist ebenfalls schneller beendet, führt jedoch zu keiner hohen Lichtemission. Dies reflektiert einen Unterschied in der Emitterstärke, denn das Hauptprodukt ist offensichtlich kynurischer Natur und sollte daher über ein Dioxetan gebildet werden.

Die Oxidation von 6-Methoxymelatonin wird hauptsächlich durch eine Hämin-katalysierte Reaktion mit Superoxidanionen hervorgerufen. Die Hydroxylradikale spielen eine nur untergeordnete Rolle, da es durch den ${ }^{\circ} \mathrm{OH}-$ Fänger DMSO (Abb. 25) kaum zu einer Verminderung der Lumineszenz kommt. Im Gegensatz dazu führt der Superoxidanionenfänger Tiron (Abb. 26) zu einer fast vollständigen Inhibition.

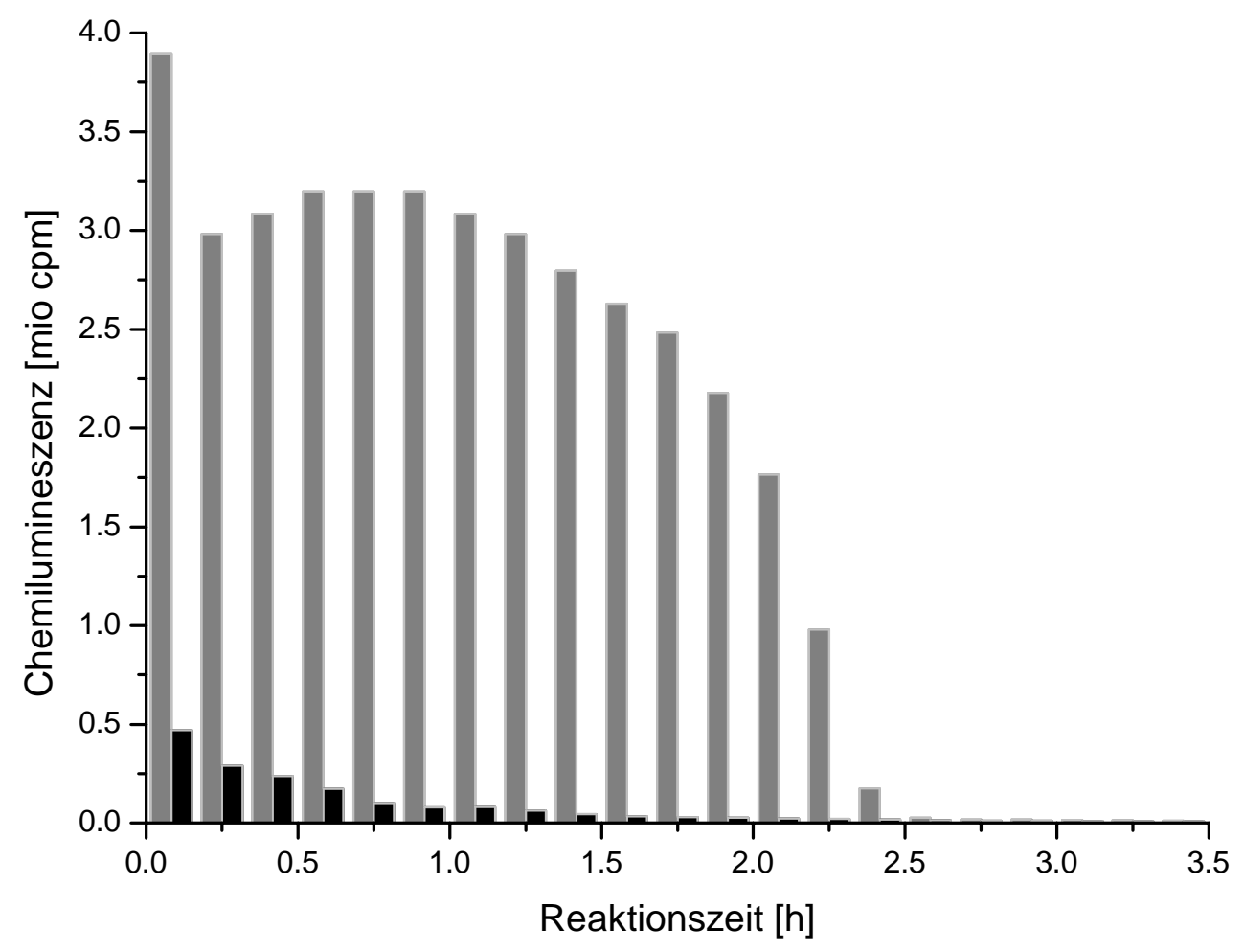

Abb. 21: Lumineszenzmessung der Oxidation von Melatonin und 6-Methoxymelatonin in einem Hämin-katalysierten $\mathbf{H}_{2} \mathrm{O}_{2}$-System im Vergleich. Graue Balken: Melatonin; schwarze Balken: 6-Methoxymelatonin. (Messverfahren siehe 2.4) 


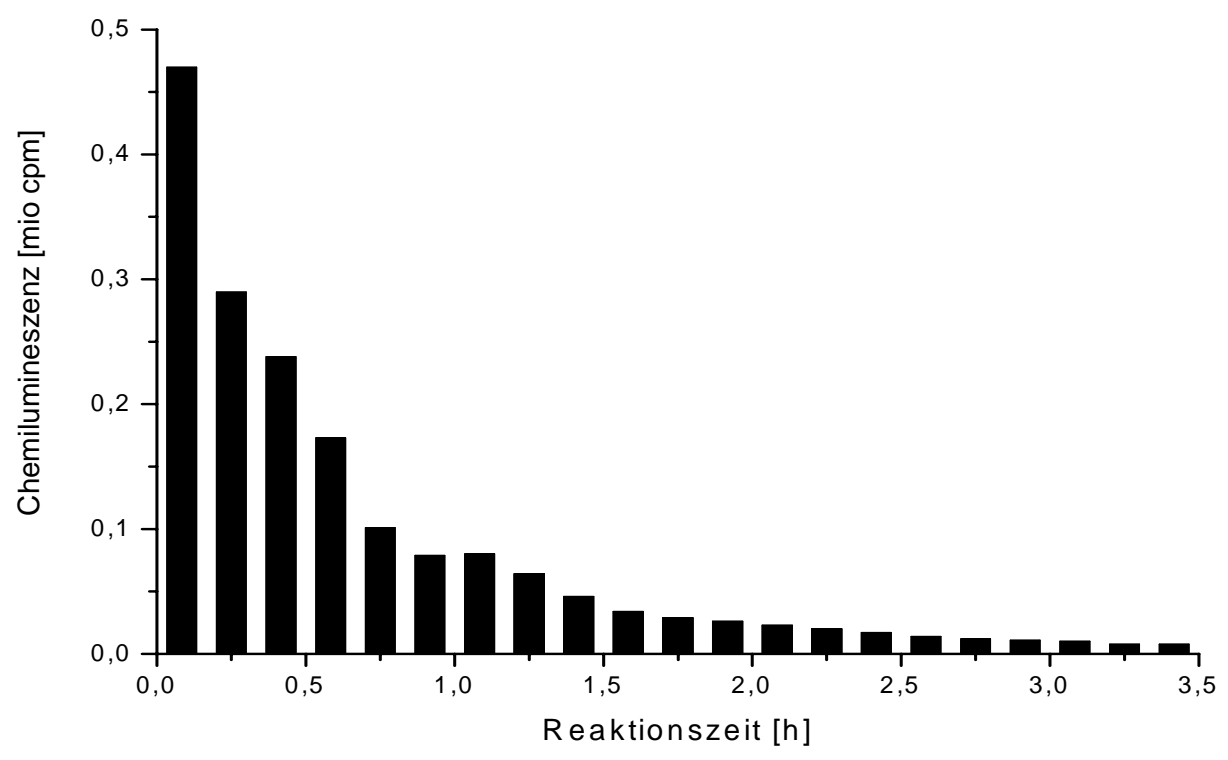

Abb. 22: Lumineszenzmessung der Oxidation von 6-Methoxymelatonin in einem Häminkatalysierten $\mathrm{H}_{2} \mathrm{O}_{2}$-System. 6-Methoxymelatonin $400 \mu \mathrm{M}$, Hämin- $\mathrm{Cl} 5 \mu \mathrm{M}, \mathrm{H}_{2} \mathrm{O}_{2} 1 \%$. (Endkonzentrationen)

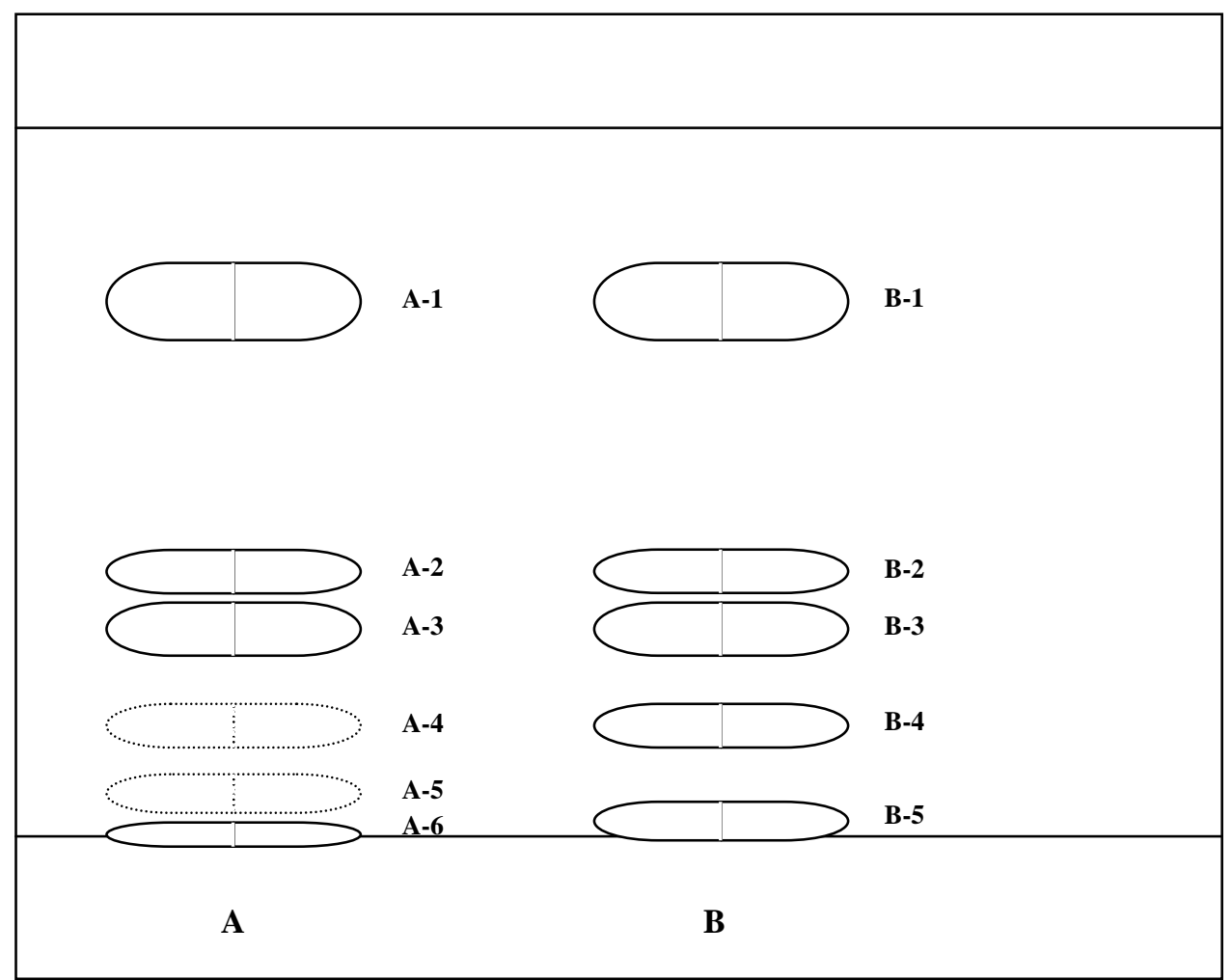

Abb. 23: Dünnschichtchromatograhie von 6-Methoxymelatonin und dessen Oxidationsprodukten in einem Hämin-katalysierten $\mathrm{H}_{2} \mathrm{O}_{2}$ System (Experiment 1). Laufmittel: Ethylacetat / MetOH (9:1); Laufstrecke 14,4 cm; Spur A: 6-Methoxymelatonin $400 \mu \mathrm{M}$, Hämin-Cl 5 $\mu \mathrm{M}, \mathrm{H}_{2} \mathrm{O}_{2} 1 \%$; Spur B: 6-Methoxymelatonin $400 \mu \mathrm{M}$, Hämin $5 \mu \mathrm{M}, \mathrm{H}_{2} \mathrm{O}_{2}$ $1 \%$, Tiron $2 \mathrm{mM}$; 
Tab. 8: Charakterisierung der Banden aus Abb. 23

\begin{tabular}{|c|l|c|l|c|}
\hline Bande & \multicolumn{1}{|c|}{ Spur A } & $\begin{array}{c}\text { Rf- } \\
\text { Wert }\end{array}$ & \multicolumn{1}{|c|}{ Spur B } & $\begin{array}{c}\text { Rf- } \\
\text { Wer } \\
\mathrm{t}\end{array}$ \\
\hline 1 & $254+$ & 0,75 & $254++$ & 0,75 \\
\hline 2 & $\begin{array}{l}254+ \\
6-M e t h o x y m e l a t o n i n\end{array}$ & 0,36 & $\begin{array}{l}254+ \\
\text { 6-Methoxymelatonin }\end{array}$ & 0,36 \\
\hline 3 & $($ AFDK) 366+++ & 0,28 & $($ ADFK) 366 (+) & 0,28 \\
\hline 4 & $366(+)$ & 0,16 & $366(+)$ & 0,16 \\
\hline 5 & $366(+)$ & 0,05 & $366(+)$ & 0,02 \\
\hline 6 & 366 rötlich & 0,00 & & \\
\hline
\end{tabular}

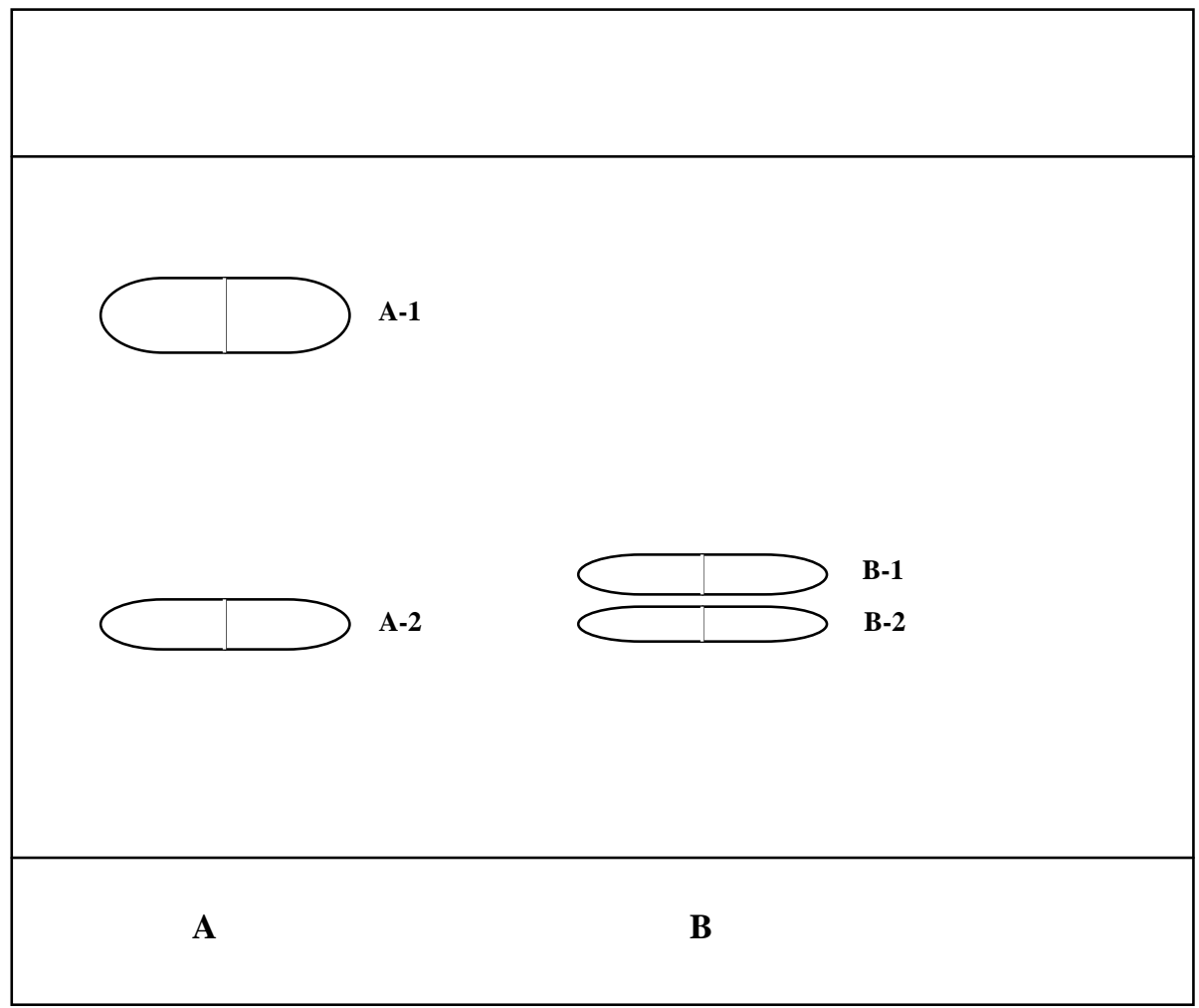

Abb. 24: Dünnschichtchromatograhie von 6-Methoxymelatonin und dessen Oxidationsprodukten in einem Hämin-katalysierten $\mathbf{H}_{2} \mathbf{O}_{2}$-System (Experiment 2). Laufmittel: Ethylacetat / MetOH (9:1); Laufstrecke 13,8 cm; Spur A: 6-Methoxymelatonin $400 \mu \mathrm{M}$, Hämin-Cl $5 \mu \mathrm{M}, \mathrm{H}_{2} \mathrm{O}_{2} 1 \%$; Spur B: 6-Methoxymelatonin Standard; 
Tab. 9: Charakterisierung der Banden aus Abb. 24

\begin{tabular}{|c|l|c|l|c|}
\hline Bande & \multicolumn{1}{|c|}{ Spur A } & $\begin{array}{c}\text { Rf- } \\
\text { Wert }\end{array}$ & \multicolumn{1}{|c|}{ Spur B } & $\begin{array}{c}\text { Rf- } \\
\text { Wert }\end{array}$ \\
\hline 1 & $254+$ & 0,76 & $\begin{array}{l}254+\text { (bläulich) } \\
\text { 6-Methoxymelatonin }\end{array}$ & 0,39 \\
\hline 2 & $366++++$ & 0,31 & $366(+)$ & 0,31 \\
\hline
\end{tabular}

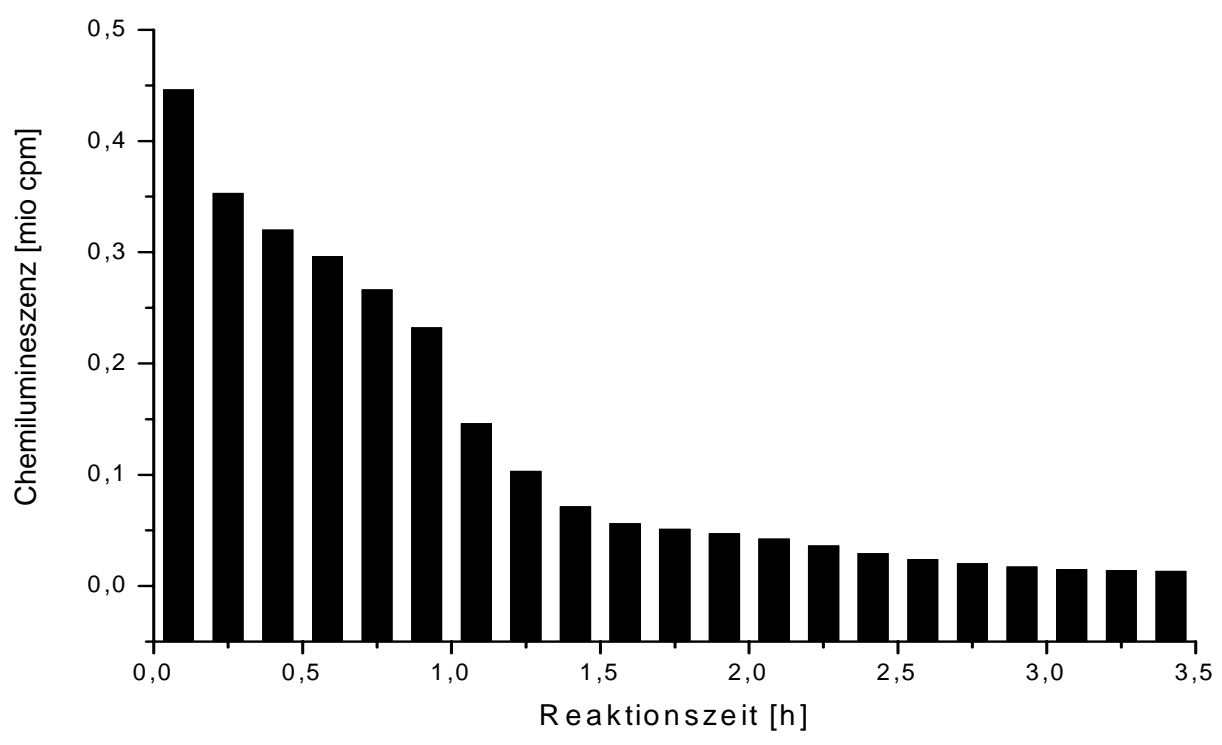

Abb. 25: Lumineszenzmessung der Oxidation von 6-Methoxymelatonin in einem Häminkatalysierten $\mathrm{H}_{2} \mathrm{O}_{2}$-System in Gegenwart von DMSO. 6-Methoxymelatonin $400 \mu \mathrm{M}$, Hämin-Cl $5 \mu \mathrm{M}, \mathrm{H}_{2} \mathrm{O}_{2}$ 1\%, DMSO $10 \%$. (Endkonzentrationen)

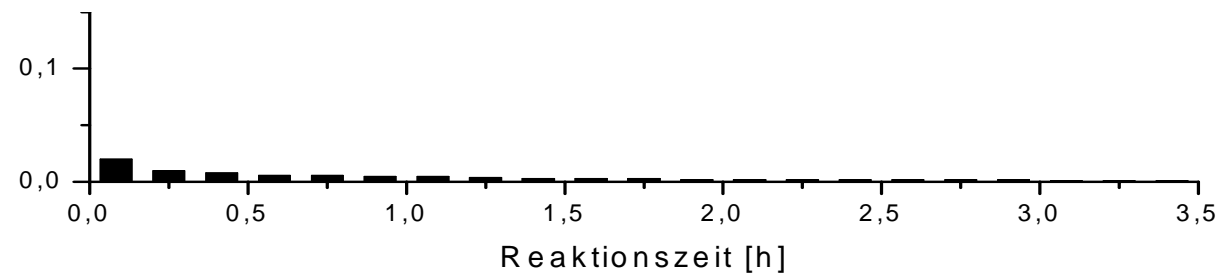

Abb. 26: Lumineszenzmessung der Oxidation von 6-Methoxymelatonin in einem Häminkatalysierten $\mathrm{H}_{2} \mathrm{O}_{2}$-System in Gegenwart von Tiron. 6-Methoxymelatonin $400 \mu \mathrm{M}$, Hämin- $\mathrm{Cl} 5 \mu \mathrm{M}, \mathrm{H}_{2} \mathrm{O}_{2} 1 \%$, Tiron $2 \mathrm{mM}$. (Endkonzentrationen) 
Seite 311 


\subsection{Oxidation mit ABTS-Kationradikalen}

Die stöchiometrische Auswertung von Melatonin (Abb. 27, 28) dokumentiert, dass mit der Abnahme des Verhältnisses von Melatonin zu ABTS-Kationradikalen (1:5, 1:10, 1:20 und 1:40) eine Steigerung der gefangenen Radikale pro Melatonin-Molekül zu erkennen ist. Hierbei steigt der Wert von ca. 4,7 bei einem Verhältnis von 1:5 auf bis zu 10 bei einem Verhältnis von 1:40.

Auch bei den strukturverwandten Indolaminen Tryptamin, 5-Methoxytryptamin, $\mathrm{N}$-Acetylserotonin und Serotonin ist eine Steigerung zu verzeichnen, jedoch mit sehr variabler Tendenz.

So zeigt $N$-Acetylserotonin (Abb. 36, 37) ein ähnliches Verhalten wie Melatonin. Bei einem Verhältnis von 1:40 werden sogar mehr als $12 \mathrm{ABTS}^{{ }^{+}}$pro NAS-Molekül gefangen, wohingegen bei 5-Methoxytryptamin (Abb. 29, 30), Tryptamin (Abb. 31, 32) und Serotonin (Abb. 33, 34) nur minimale Steigerungen zu verzeichnen sind. Das Schlusslicht stellt hierbei Serotonin dar, da es bei Abnahme des Verhältnisses von 1:5 auf 1:40 lediglich ein weiteres $\mathrm{ABTS}^{\bullet+}$ pro Serotonin-Molekül fängt.

Auch Unterschiede in der Geschwindigkeit der Reaktionen lassen sich klar erkennen.

So werden in den ersten 15 min mehr Melatonin-Moleküle oxidiert als im Vergleichszeitraum bei Tryptamin und 5-Methoxytryptamin. N-Acetyltryptamin, $N$-Acetylserotonin und Serotonin zeigen hingegen keine großen Unterschiede. 


\subsubsection{Umsatz von Melatonin mit ABTS-Kationradikalen}

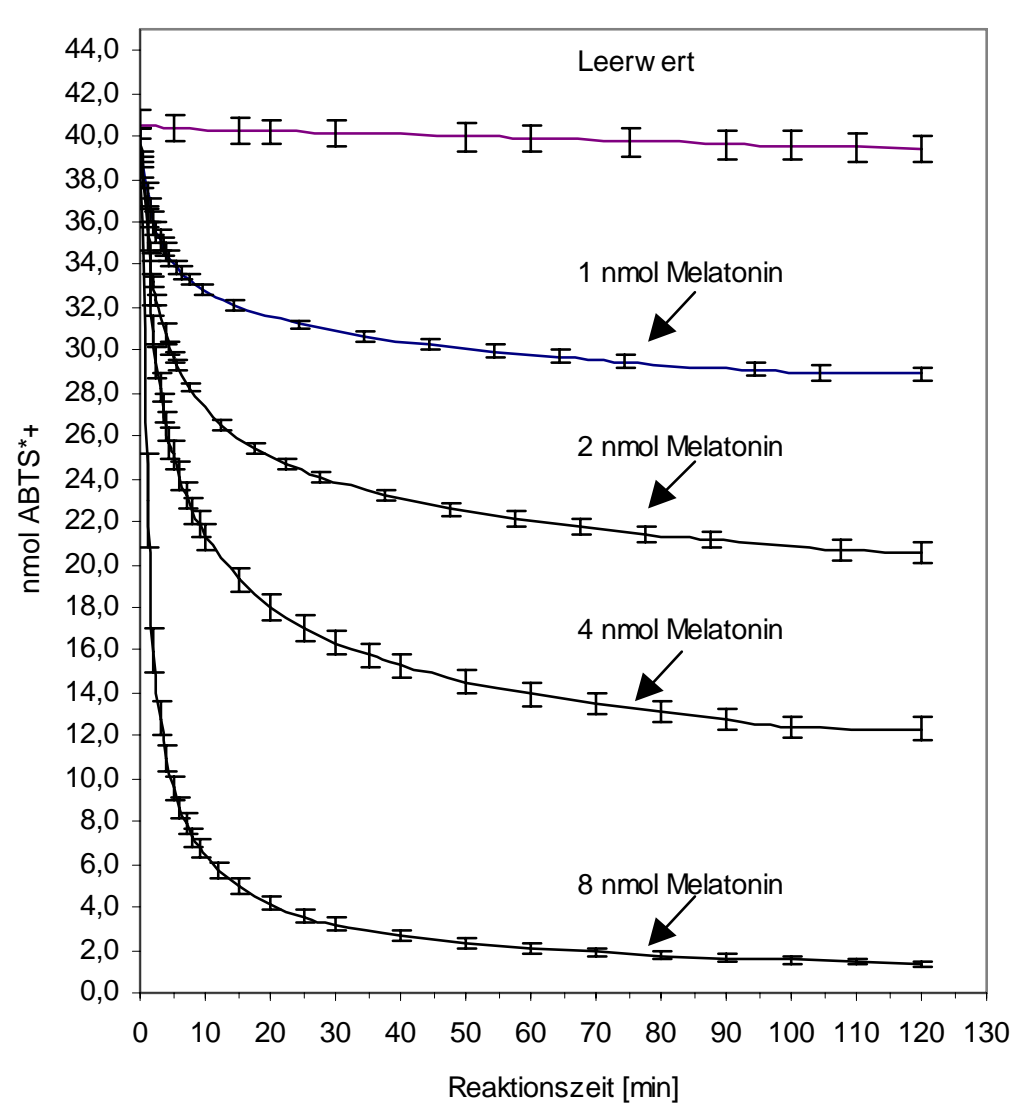

Abb. 27: Konzentrations- und Zeitabhängkeit der ABTS $^{\bullet+}$-Reduktion durch Melatonin.

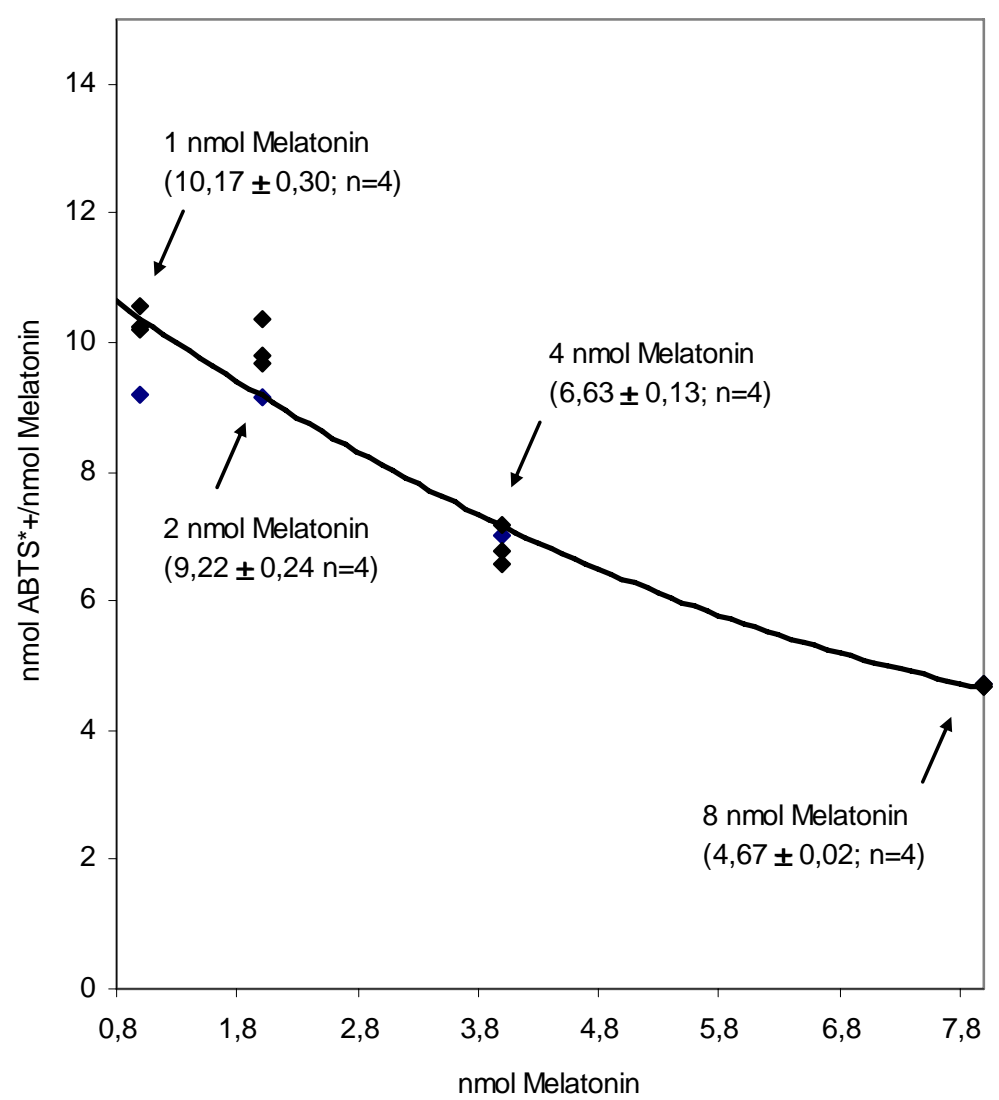

Abb. 28: Stöchiometrie des Fängers von ABTS $^{\bullet+}$ pro Melatonin-Molekül 


\subsubsection{Umsatz von 5-MT mit ABTS-Kationradikalen}

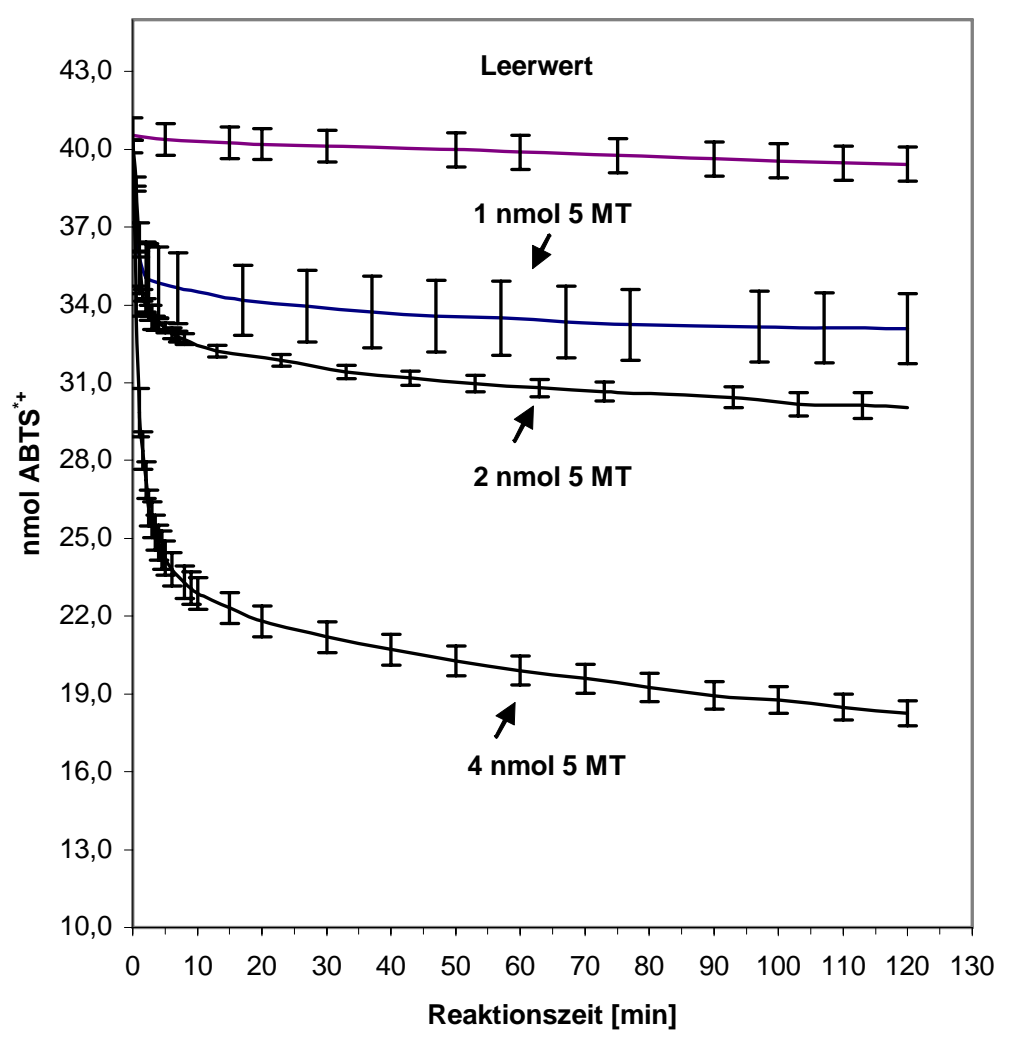

Abb. 29: Konzentrations- und Zeitabhängkeit der ABTS $^{\bullet+}$-Reduktion durch 5-Methoxytryptamin.

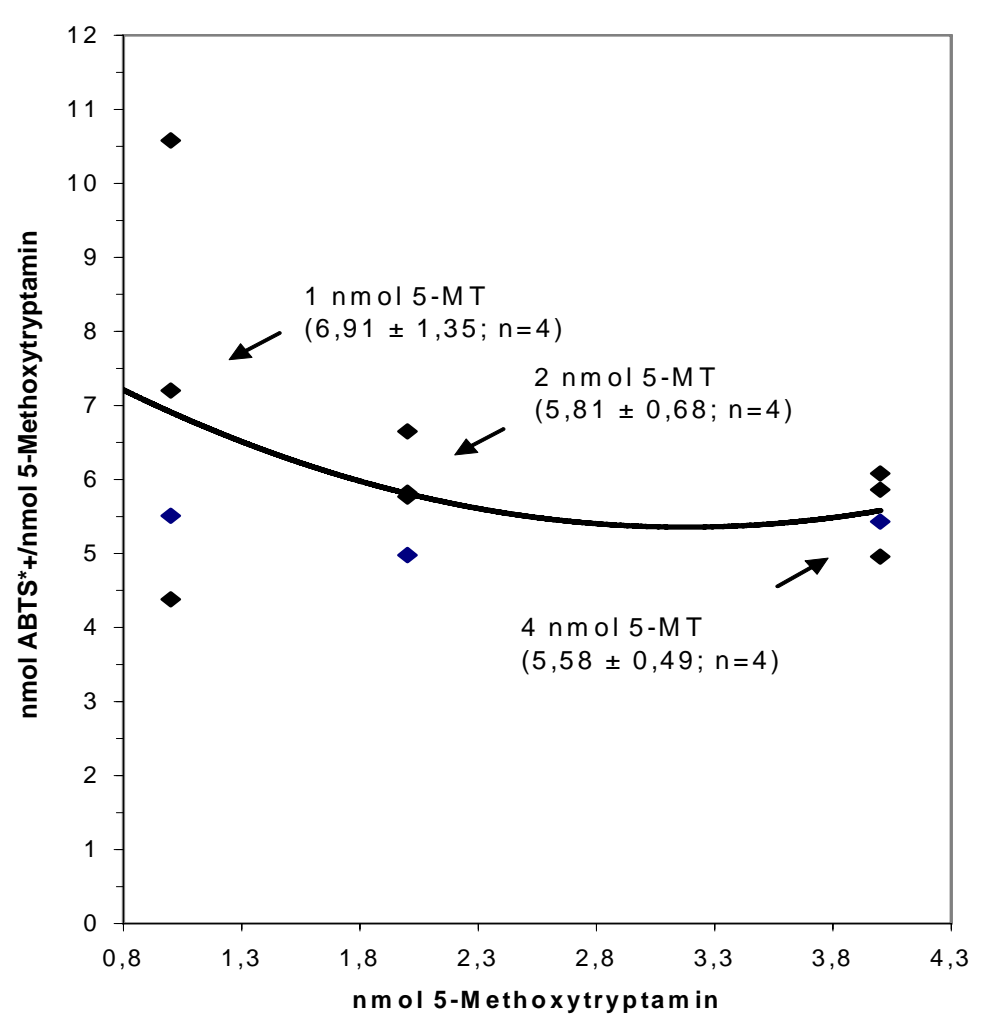

Abb. 30: Stöchiometrie des Fängers von $\mathrm{ABTS}^{\bullet+}$ pro 5-Methoxytryptamin-Molekül. 


\subsubsection{Umsatz von Tryptamin mit ABTS-Kationradikalen}

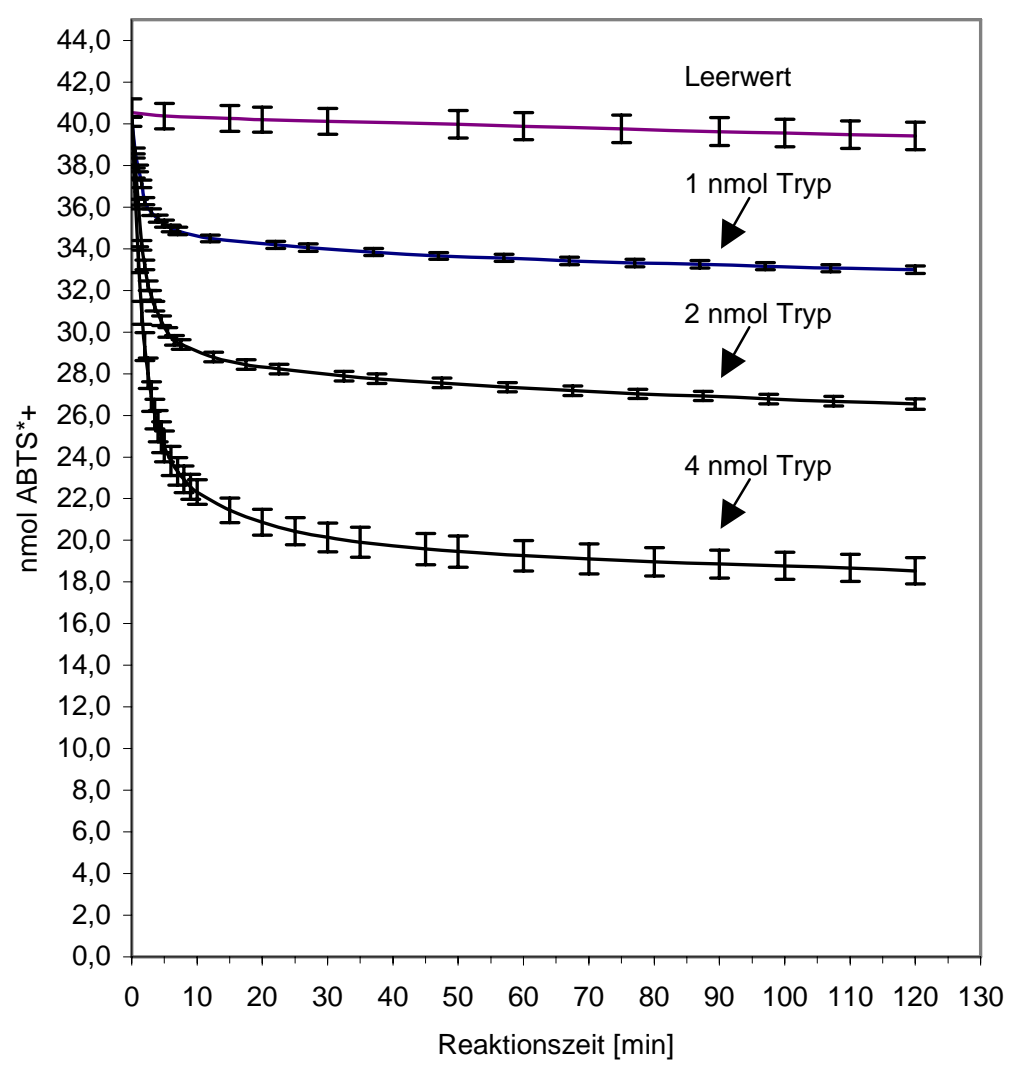

Abb. 31: Konzentrations- und Zeitabhängkeit der ABTS $^{\bullet+}$-Reduktion durch Tryptamin.

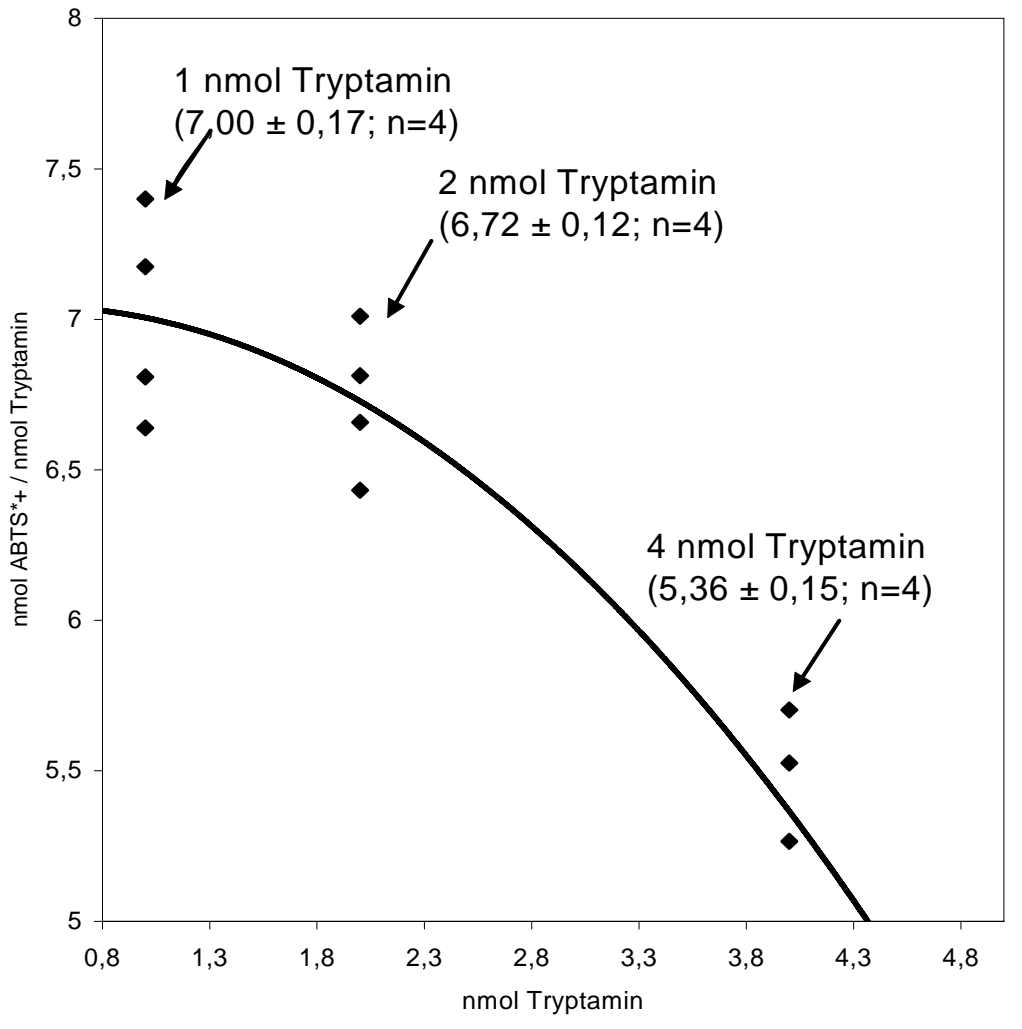

Abb. 32: Stöchiometrie des Fängers von $\mathrm{ABTS}^{\bullet+}$ pro Tryptamin-Molekül. 


\subsubsection{Umsatz von Serotonin mit ABTS-Kationradikalen}

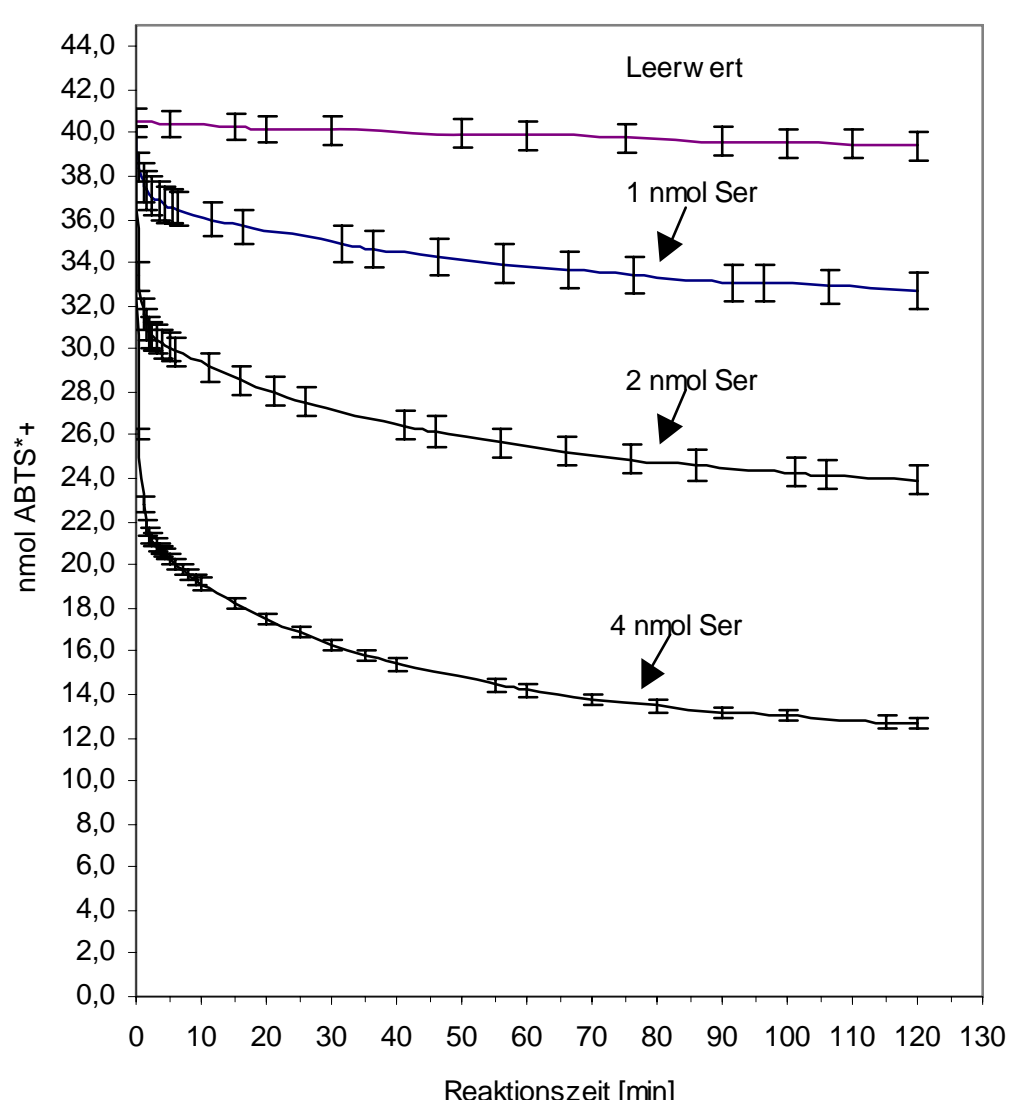

Abb. 33: Konzentrations- und Zeitabhängkeit der ABTS $^{\circ+}$-Reduktion durch Serotonin.

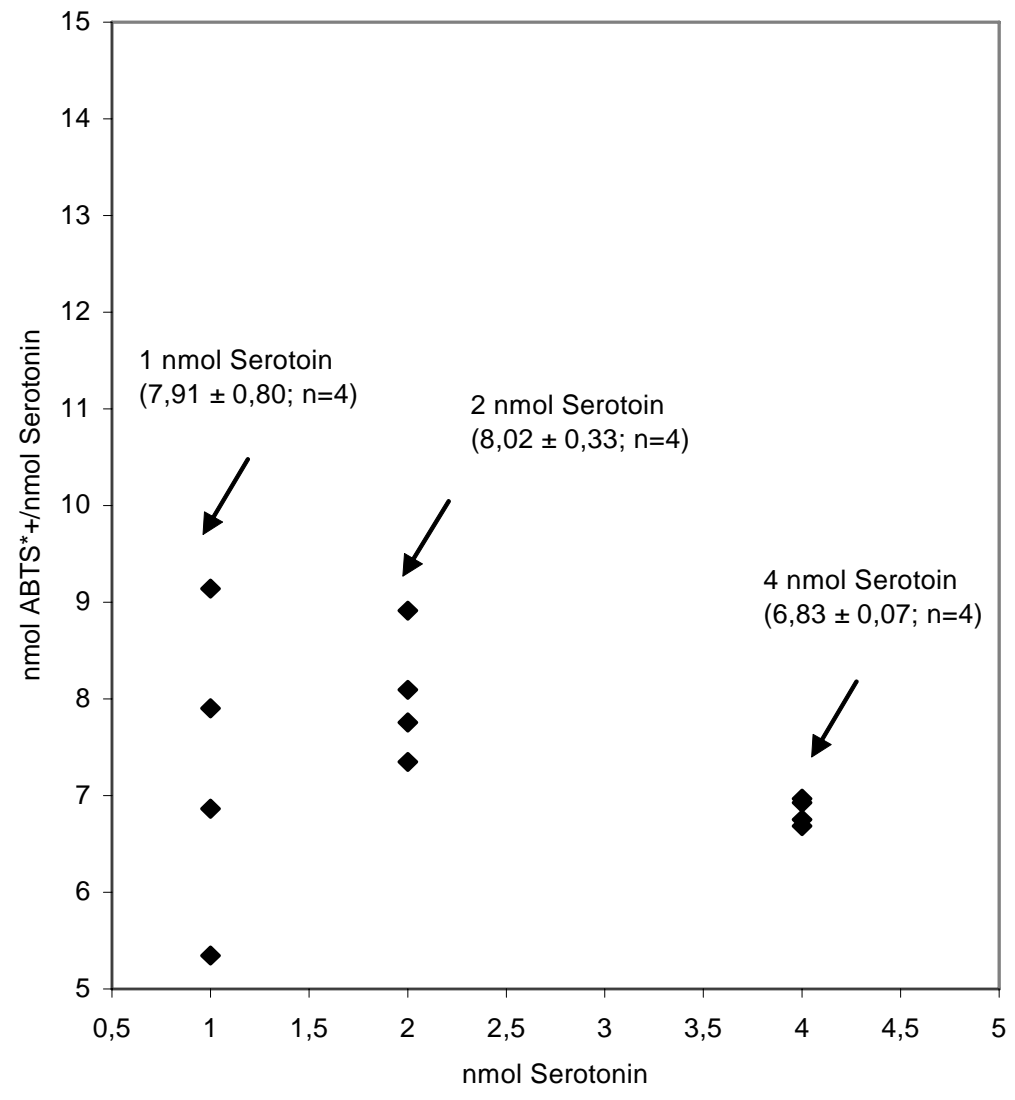

Abb. 34: Stöchiometrie des Fängers von ABTS $^{\bullet+}$ pro Serotonin-Molekül. 


\subsubsection{Umsatz von NAT mit ABTS-Kationradikalen}

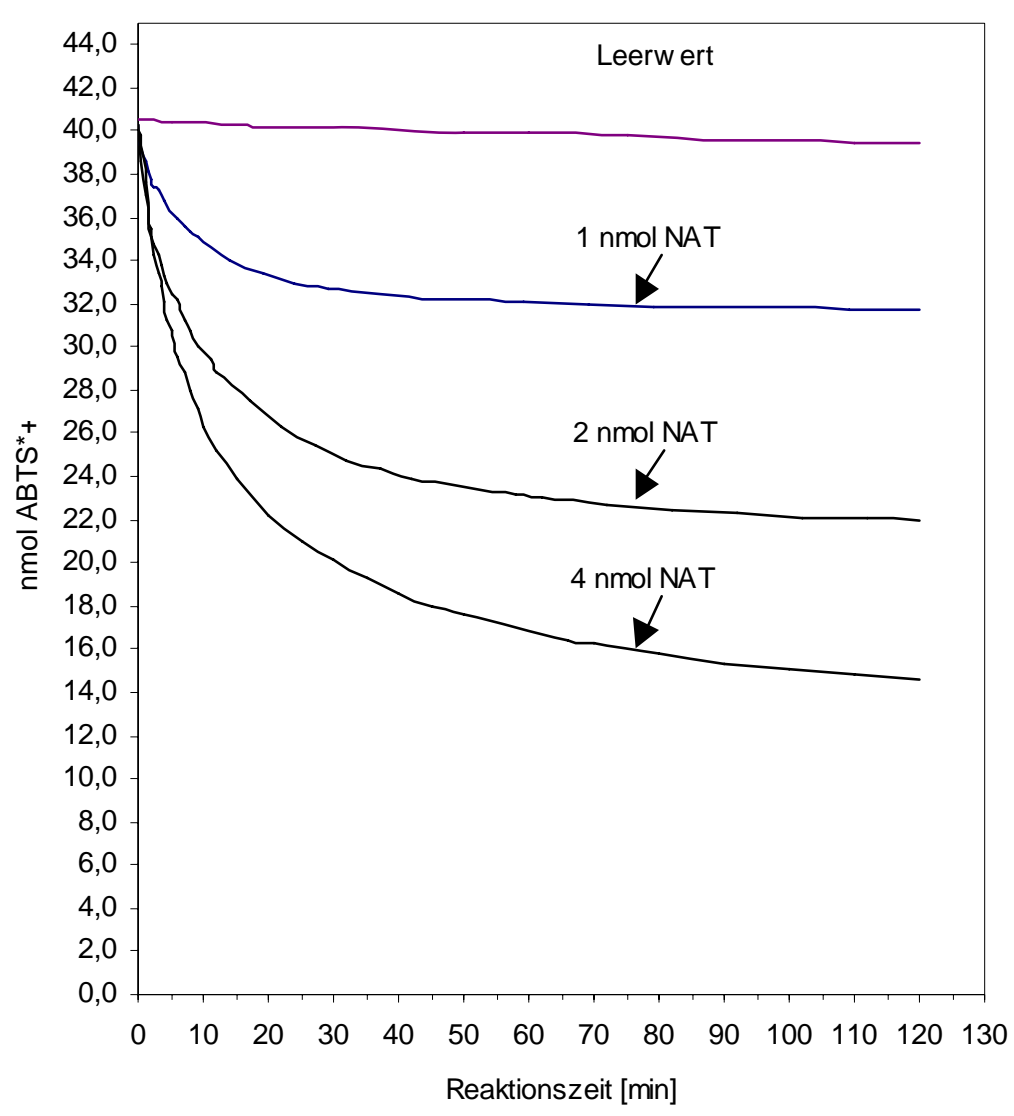

Abb. 35: Konzentrations- und Zeitabhängkeit der ABTS $^{\bullet+}$-Reduktion durch $N$-Acetyltryptamin. 


\subsubsection{Umsatz von NAS mit ABTS-Kationradikalen}

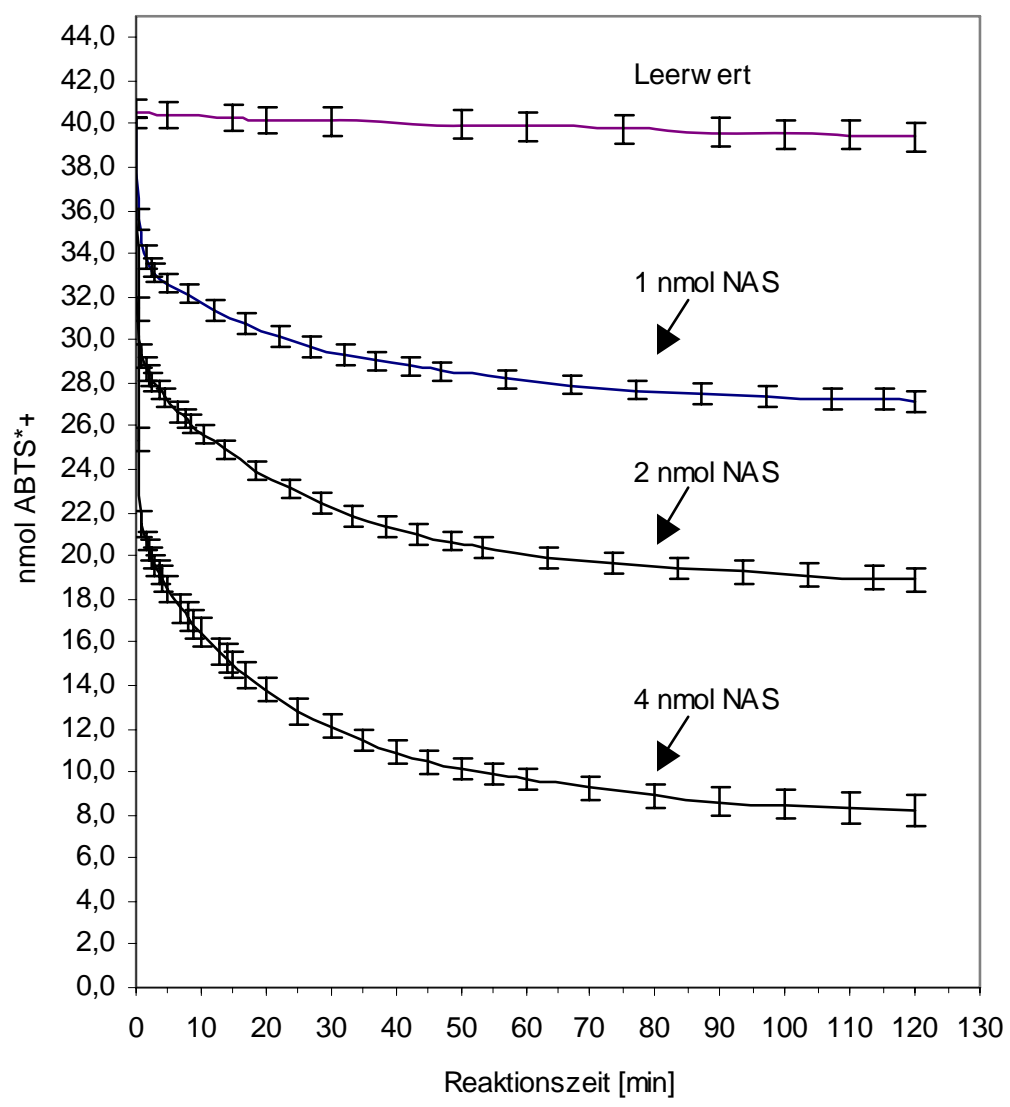

Abb. 36: Konzentrations- und Zeitabhängkeit der ABTS $^{\bullet+}$-Reduktion durch $N$-Acetylserotonin.

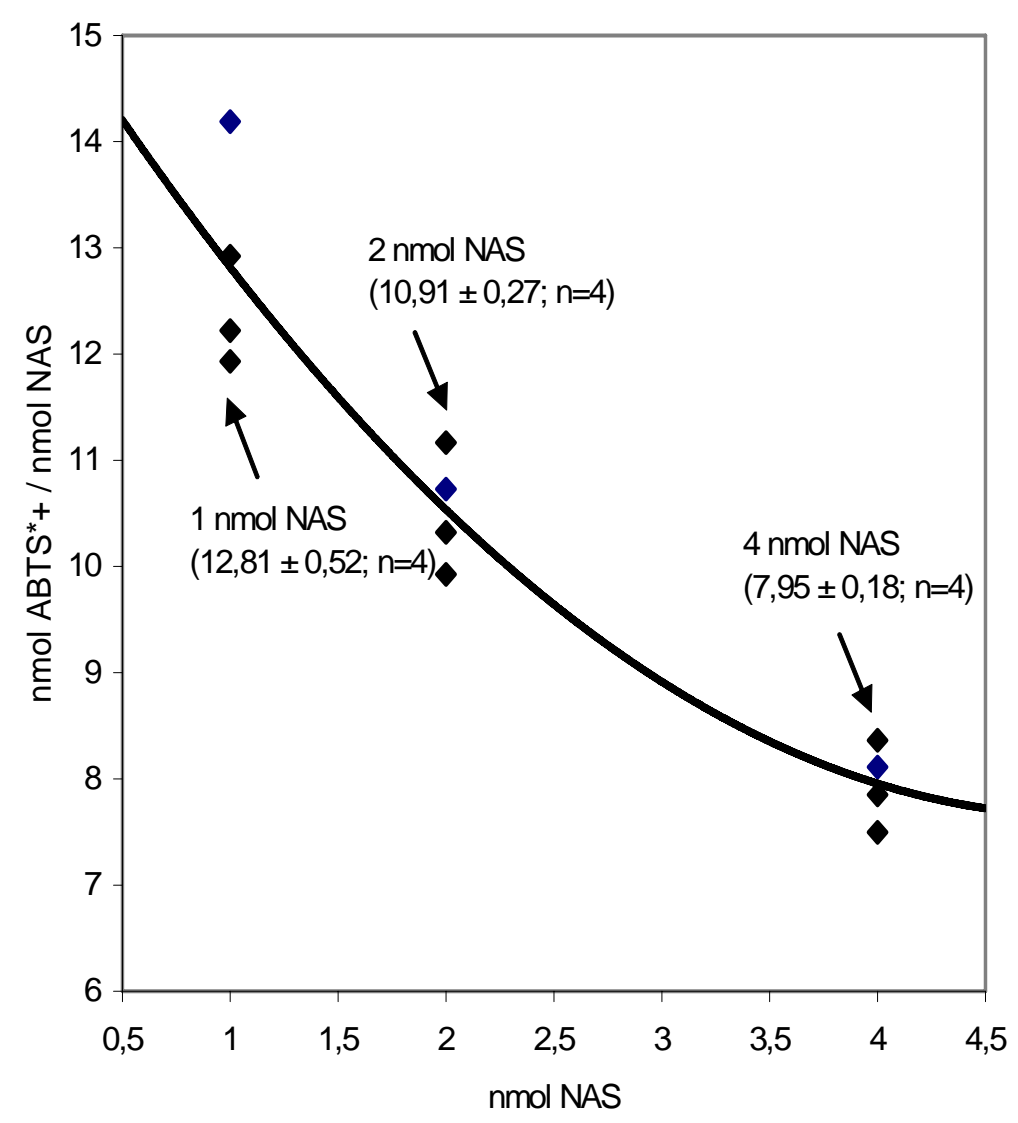

Abb. 37: Stöchiometrie des Fängers von $\mathrm{ABTS}^{\bullet+}$ pro $N$-Acetylserotonin-Molekül. 


\section{$\underline{3.4}$ Produkte der ABTS-Kationradikalreaktion}

In der Dissertation von Dun-Xian Tan (2002) wurde die Hypothese aufgestellt, dass Melatonin insgesamt 4 ABTS-Kationradikale verbraucht. Bei einem Überschuß dieser Radikale wird jedoch eine höhere Anzahl von diesen verbraucht (Abb. 28).

Beim Umsatz von Melatonin mit $\mathrm{ABTS}^{\bullet+}$ konnte im Chromatogramm (Laufmittelgemisch (BEW; 9,5:0,5) unter UV-Licht (366 nm) außer dem bekannten AFMK (Produkt 1), eine prominente Bande sehr dicht unterhalb des Melatonins entdeckt werden. Zur bestmöglichen Isolierung dieser Bande wurden unterschiedlichste Laufmittelsysteme in der DC getestet, wobei sich immer wieder andere Banden störend auf die Präparation auswirkten. Erst der Austausch von Melatonin durch AFMK erbrachte den gewünschten Erfolg (siehe Abb. $38 \mathrm{a} / \mathrm{b})$.

\section{$\underline{\text { Analyse der Produkte }}$}

Zur Isolierung und Analyse wurde gemäß 2.3.3 ein Ansatz mit AFMK als Edukt im 50fachem Volumen zur Reaktion gebracht, aufbereitet (2.4 Extraktion) und auf eine PTLC mit Ethylacetat / Methanol (9.5:0.5) gebracht. Schon bei Tageslicht konnten Bereiche mit einem schwachen Gelbschimmer voneinander unterschieden werden. Unter UV-Licht zeigten sich klare, voreinander abgegrenzte Banden. Da sich neben der bereits erwähnten Substanz (Produkt 3), welche sich im UV-Licht von $366 \mathrm{~nm}$ mit einer intensiv bläulichen Farbe darstellt, eine weitere (Produkt 2) oberhalb dieser mit einer intensiv grünlichen Farbe zeigte, wurde diese ebenfalls gemäß 2.3.3 der PTLC entnommen und aufbereitet (2.4 Extraktion) 

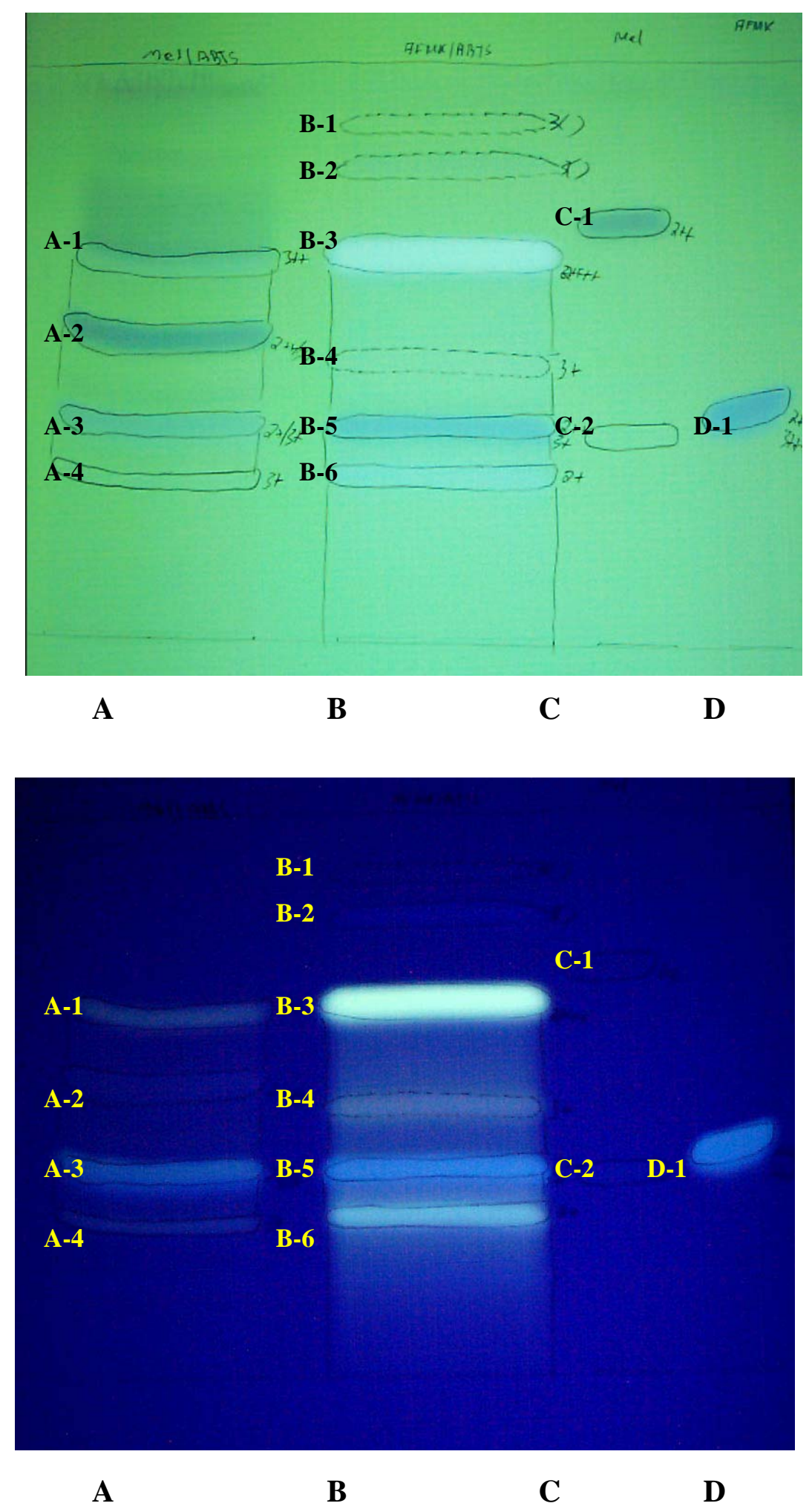

Abb. 38a/b: Vergleich des Umsatzes von Mel und AFMK mit ABTS $^{\bullet+}(254 / 366$ nm)

(Laufmittel: BEW (16 : 0,25:0,5), Laufstrecke 14,5 cm, Spur A: Melatonin $4 \mathrm{mM}$, ABTS 1,44 Ext; Spur B: AFMK 4 mM, ABTS 1,44 Ext., $\underline{\text { Spur C: }}$ : Mel Std., Spur D: AFMK Std. 
Tab. 10: Charakterisierung der Banden aus Abb. 38a/b

\begin{tabular}{|c|l|l|l|l|l|l|l|l|}
\hline Bande & \multicolumn{1}{|c|}{ Spur A } & $\begin{array}{c}\text { Rf- } \\
\text { Wert }\end{array}$ & \multicolumn{1}{|c|}{ Spur B } & $\begin{array}{c}\text { Rf- } \\
\text { Wert }\end{array}$ & \multicolumn{1}{|c|}{ Spur C } & $\begin{array}{c}\text { Rf- } \\
\text { Wert }\end{array}$ & \multicolumn{1}{|c|}{ Spur D } & $\begin{array}{c}\text { Rf- } \\
\text { Wert }\end{array}$ \\
\hline 1 & $366++$ & 0,63 & 366() & 0,89 & Melatonin & 0,71 & AFMK & 0,37 \\
\hline 2 & $254++/ 366+$ & 0,50 & 366() & 0,81 & AFMK & 0,35 & & \\
\hline 3 & $254+/ 366+$ & 0,35 & $366++++$ & 0,64 & & & & \\
\hline 4 & $366+$ & 0,26 & $366+$ & 0,46 & & & & \\
\hline 5 & & & $254++/ 366+$ & 0,35 & & & & \\
\hline 6 & & & $254+$ & 0,26 & & & & \\
\hline
\end{tabular}




\section{$\underline{\text { 3.4.1 Produkt } 2}$}

Die Bestimmung der Molekülmasse wurde durch eine HPLC-MS vorgenommen. Die höchste durch EI-MS detektierbare Masse höherer Abundanz lag bei 260.1 (Abb. 39). Im HPLCChromatogramm (Abb. 40) konnten für die Massen um 260 drei Produktmaxima detektiert werden. Für die Peaks der Retentionszeiten 12.01 und 14.28 ließen sich eindeutig die Pseudomassen [M +H] von 261 nachweisen (Produkte in der ESI-MS, 2a und 2b; Abb. 4144). Die Absorptionsspektren sind in der Abb. 42 bzw. 44 wiedergegeben.

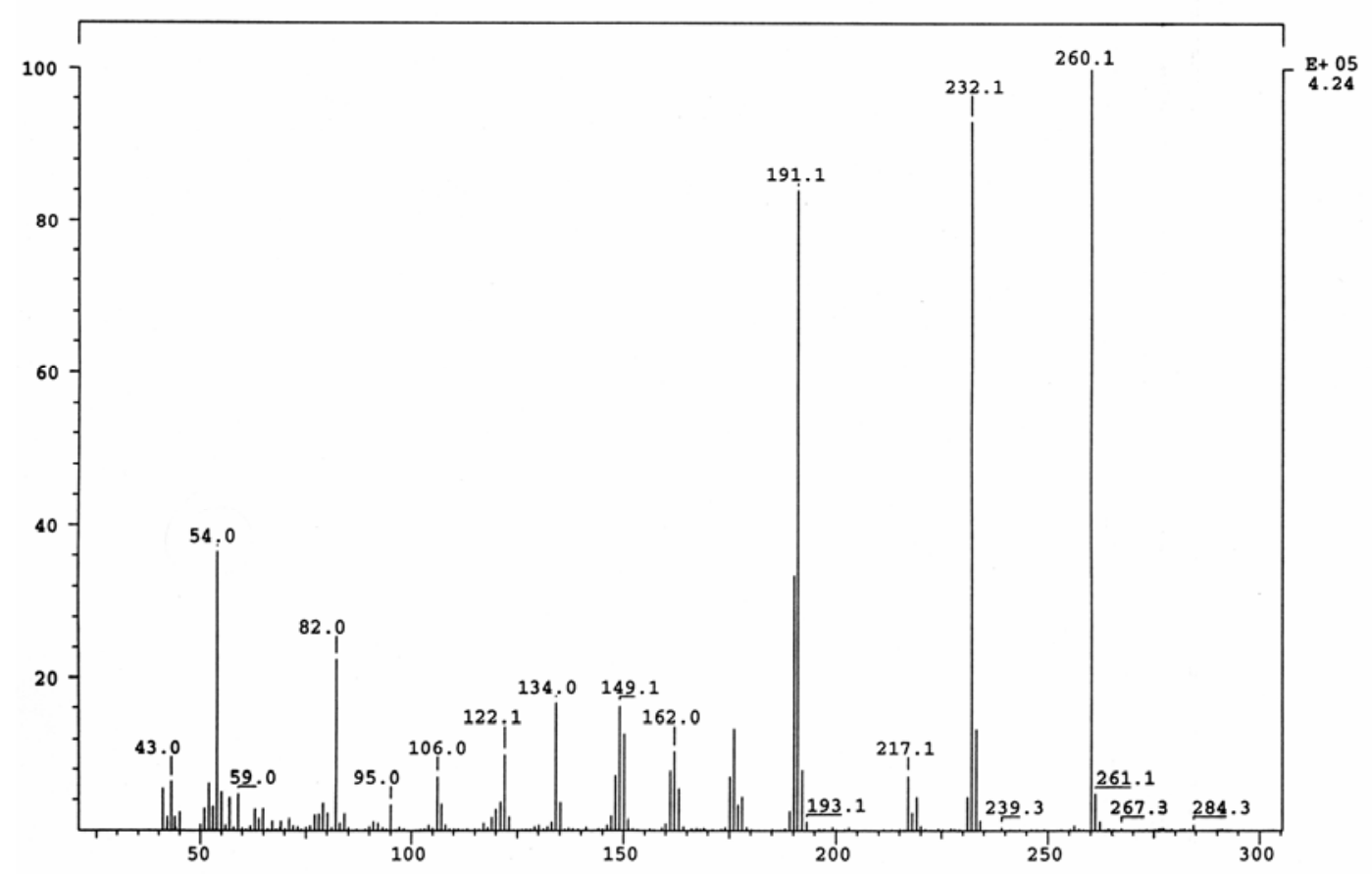

Abb. 39: Massenspektrum (EI-MS, 70 eV) von Produkt 2

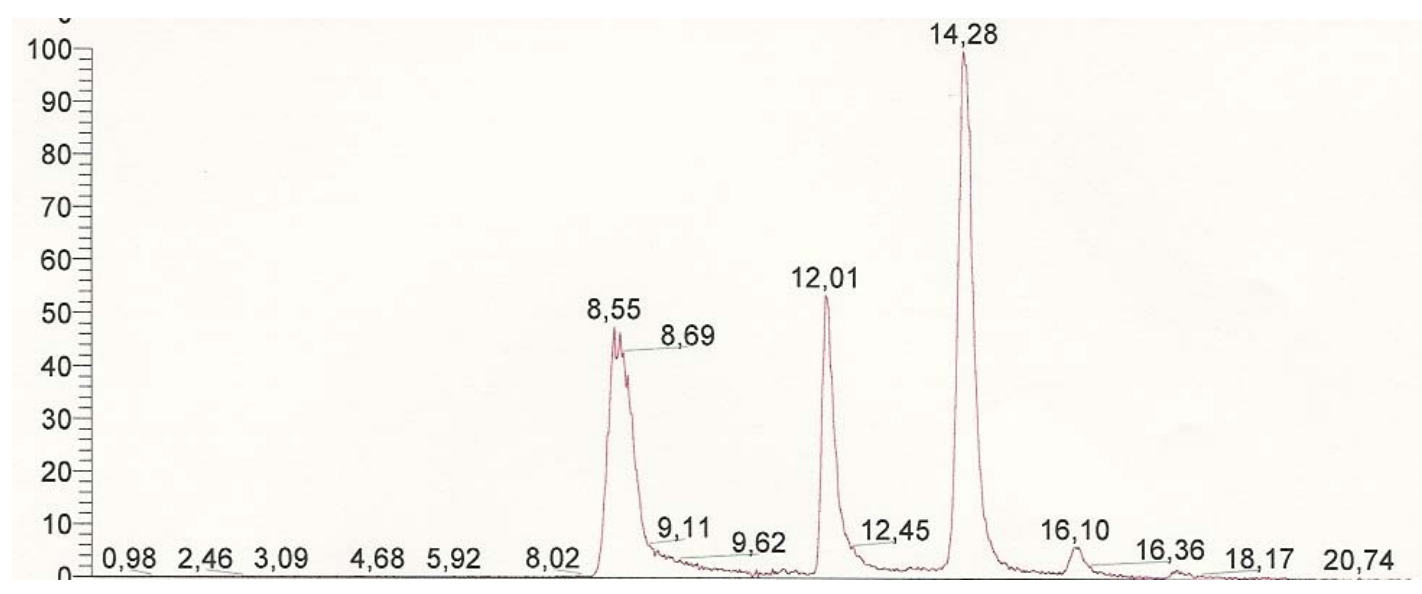

Abb. 40: HPLC-MS ( $\mathrm{MeOH} / \mathrm{H}_{2} \mathrm{O}, 10-100$ \% MeOH, 0-20 min.) von Produkt 2 


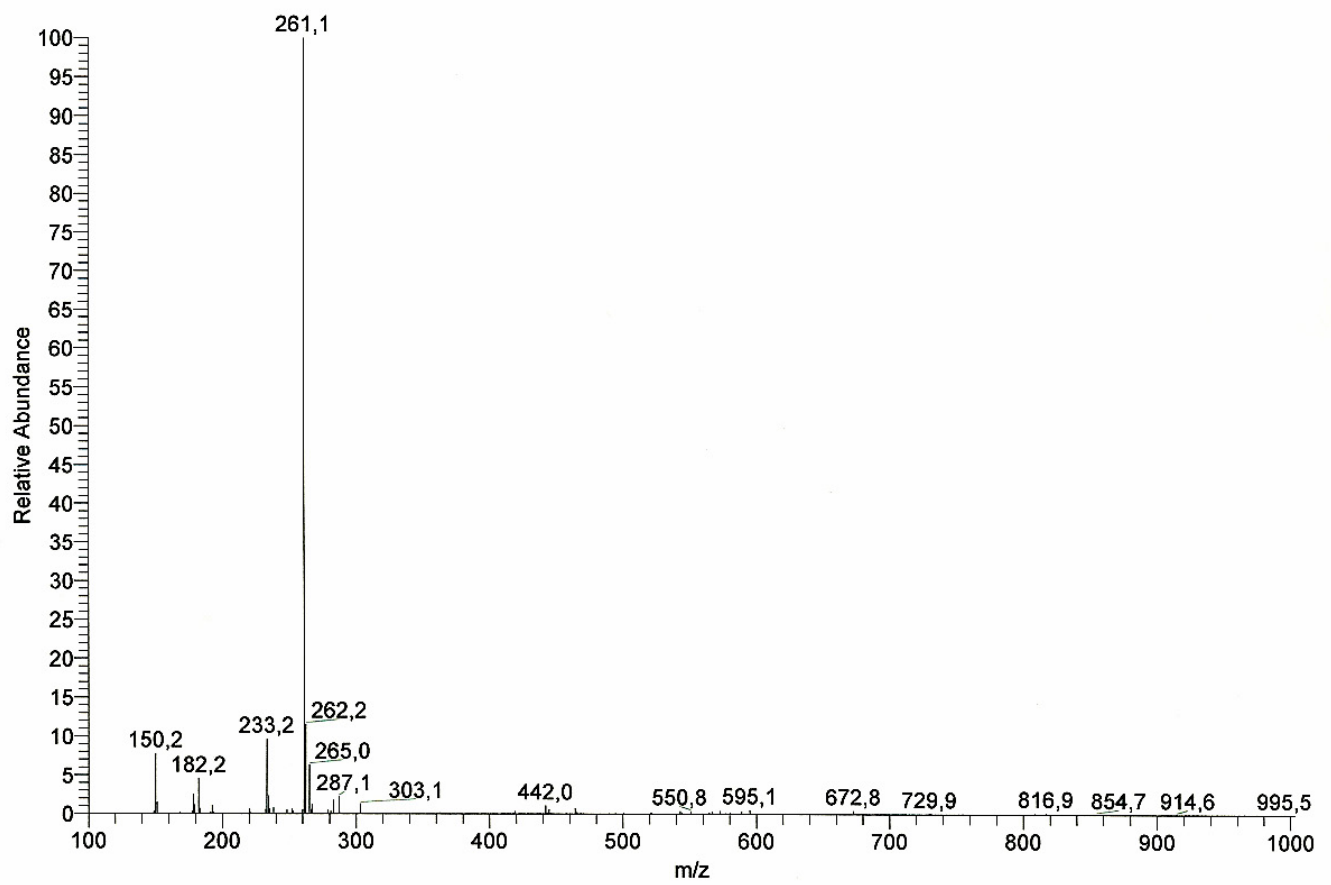

Abb. 41: Massenspektrum (ESI-HRMS, $70 \mathrm{eV}$ ) von Produkt 2a (H+)

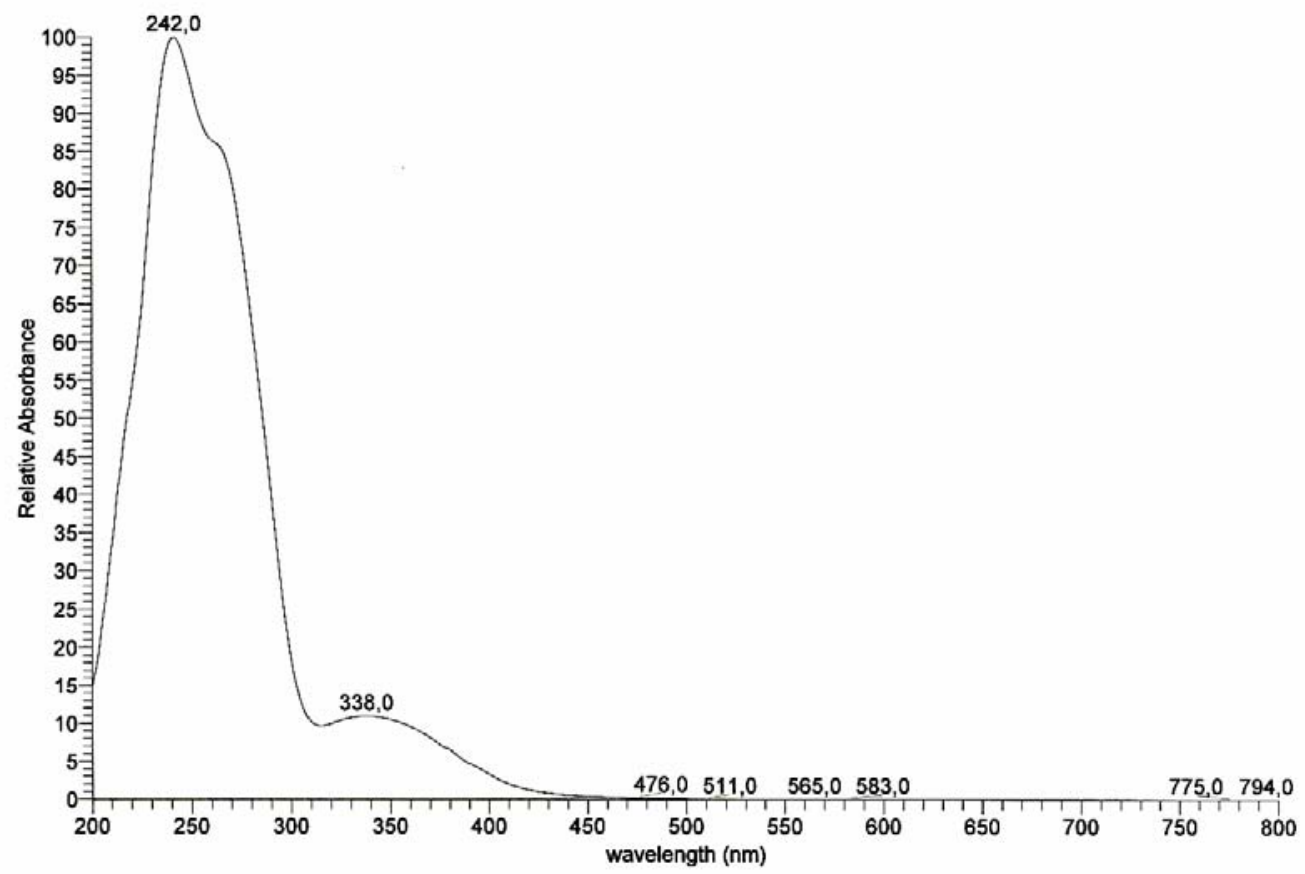

Abb. 42: UV-Spektrum von Produkt 2a 


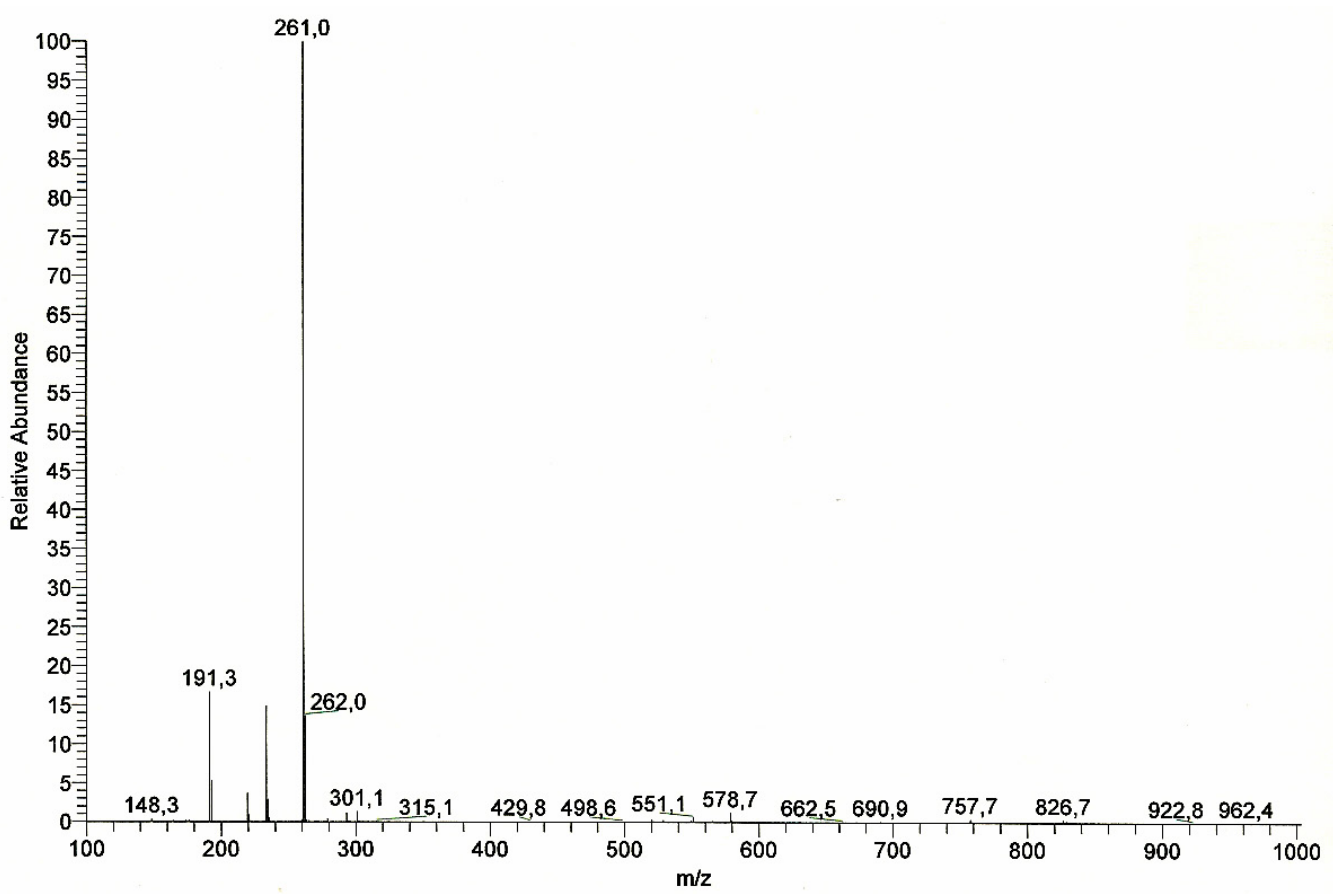

Abb. 43: Massenspektrum (ESI-HRMS, $70 \mathrm{eV})$ von Produkt 2b (H+)

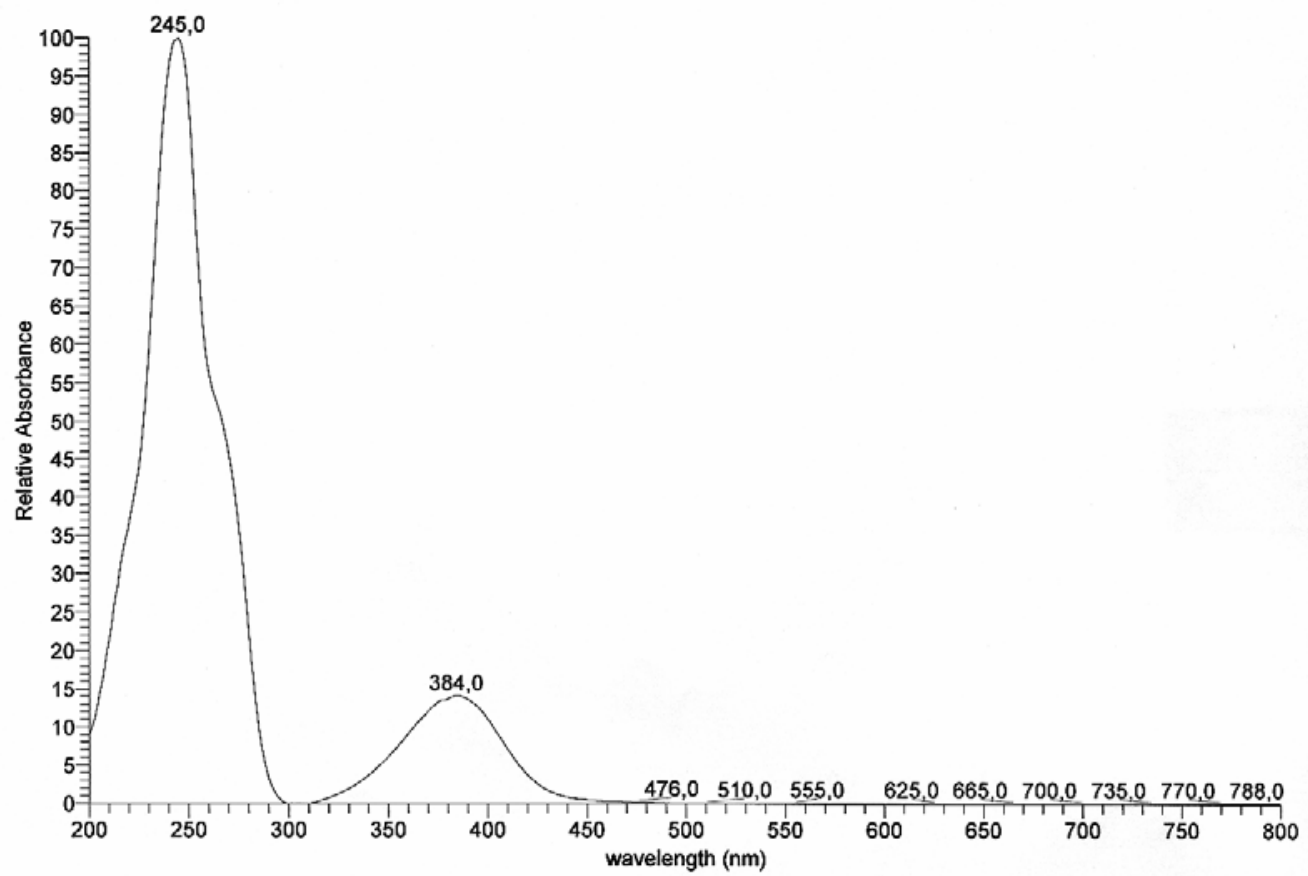

Abb. 44: UV-Spektrum von Produkt 2b

Im UV-Spektrum von Produkt 2a/b (Abb. 42, 44) treten jeweils zwei Maxima auf (Produkt 2a: $\lambda_{\max }$ 242, $338 \mathrm{~nm}$ und Produkt $2 \mathrm{~b} \lambda_{\max }$ 245, $384 \mathrm{~nm}$ ), welche typische Regionen für aromatische Ringsysteme darstellen. 
Die Produkte 2a/b wurden zur Strukturaufklärung durch ${ }^{1} \mathrm{H}-\mathrm{NMR}$ (Abb. 45), ${ }^{13} \mathrm{C}-\mathrm{NMR}$ (Abb. 46) und HMBC-Korrelation (Abb. 47) weiter analysiert. Die Spektraldaten sind in numerischer Form in Tab. 11 dargestellt.

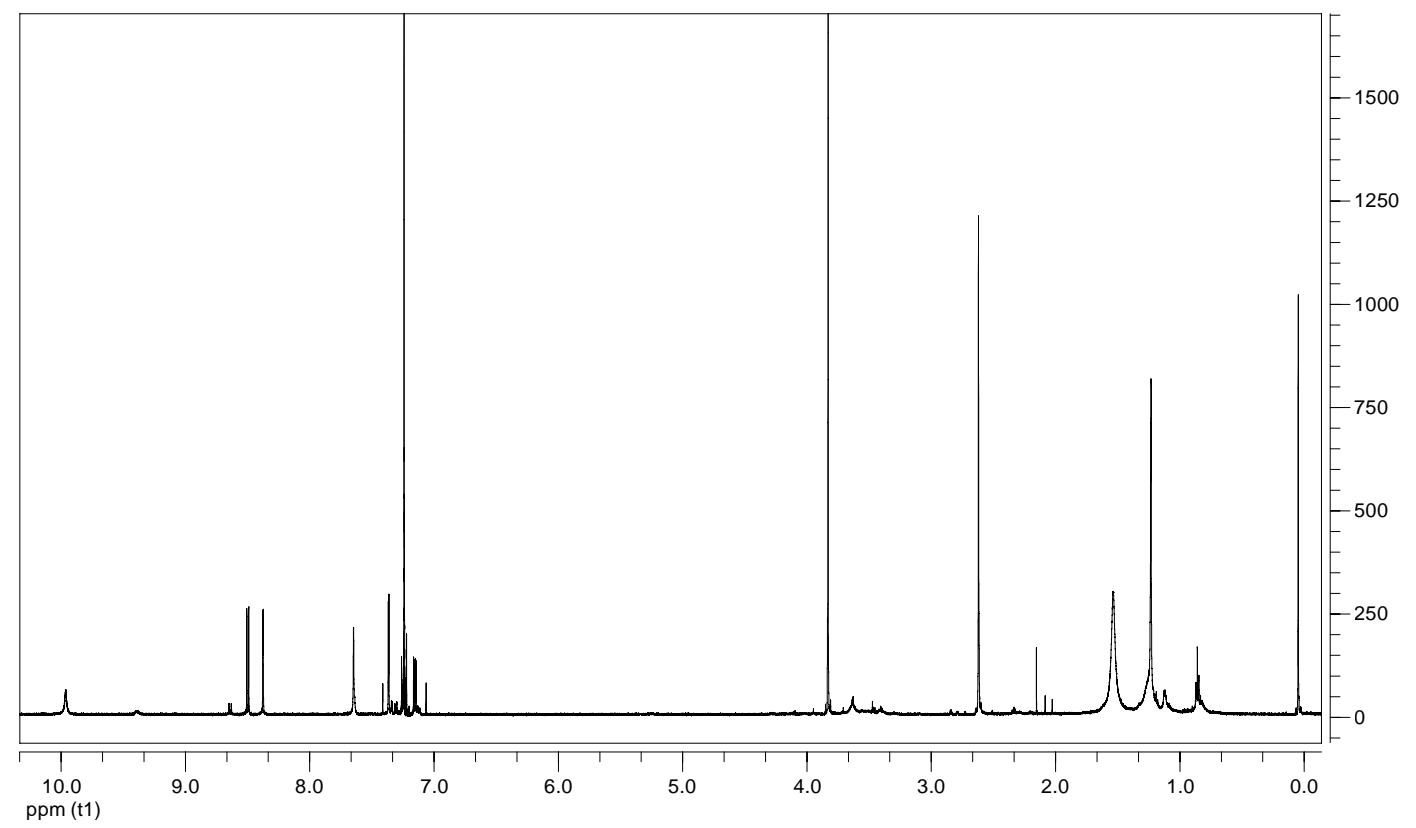

Abb. 45: ${ }^{1} \mathrm{H}-\mathrm{NMR}-S p e k t r u m ~\left(599,1 \mathrm{MHz}\right.$ ) von Produkt 2a/b in $\mathrm{CDCl}_{3}$

Das Singulett bei $\delta_{\mathrm{H}} 3.83$ mit der Intensität von 3 Protonen deutet auf eine Methoxygruppe hin. Das Singulett bei $\delta_{\mathrm{H}} 2.62$ mit der Intensität von 3 Protonen weist auf eine $\mathrm{CH}_{3}$-Gruppe hin. Typische Signale (Dublett) für die aromatische Region konnten bei $\delta_{\mathrm{H}} 7.15$, 7.37 und 8.50 entdeckt werden. Ein weiteres sehr breites Singulett-Signal bei $\delta_{\mathrm{H}} 7.65$ deutete auf eine Doppelbindung hin. 


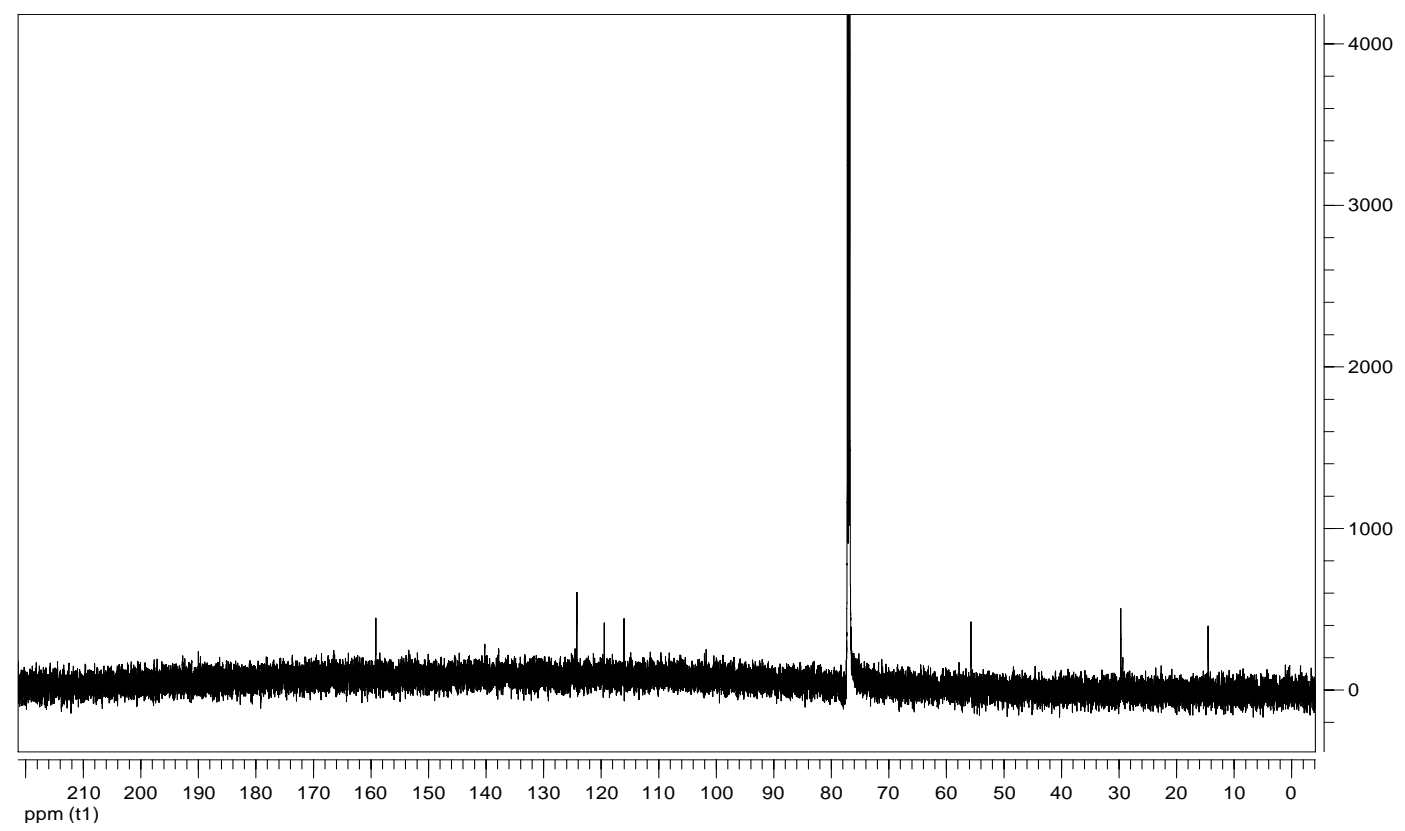

Abb. 46: ${ }^{13} \mathrm{C}-\mathrm{NMR}-S p e k t r u m ~(150,8 \mathrm{Mhz})$ von Produkt 2a/b in $\mathrm{CDCl}_{3}$

Das C-Spektrum $\left(\mathrm{CD}_{3} \mathrm{Cl}\right)$ von Produkt 2a/b zeigt nur 9 Kohlenstoff-Signale. Die aliphatische Region bei $\delta_{\mathrm{C}} 14.5$ ein klares Methylen-Signal und bei $\delta_{\mathrm{C}} 55.7$ ein Methyl-Signal. Vier quaternäre Signale konnten zu Carboxylgruppen zugeordnet werden.

Für die Auffindung der fehlenden C-Atome wurde das HMBC-Spektrum zur Hilfe gezogen.

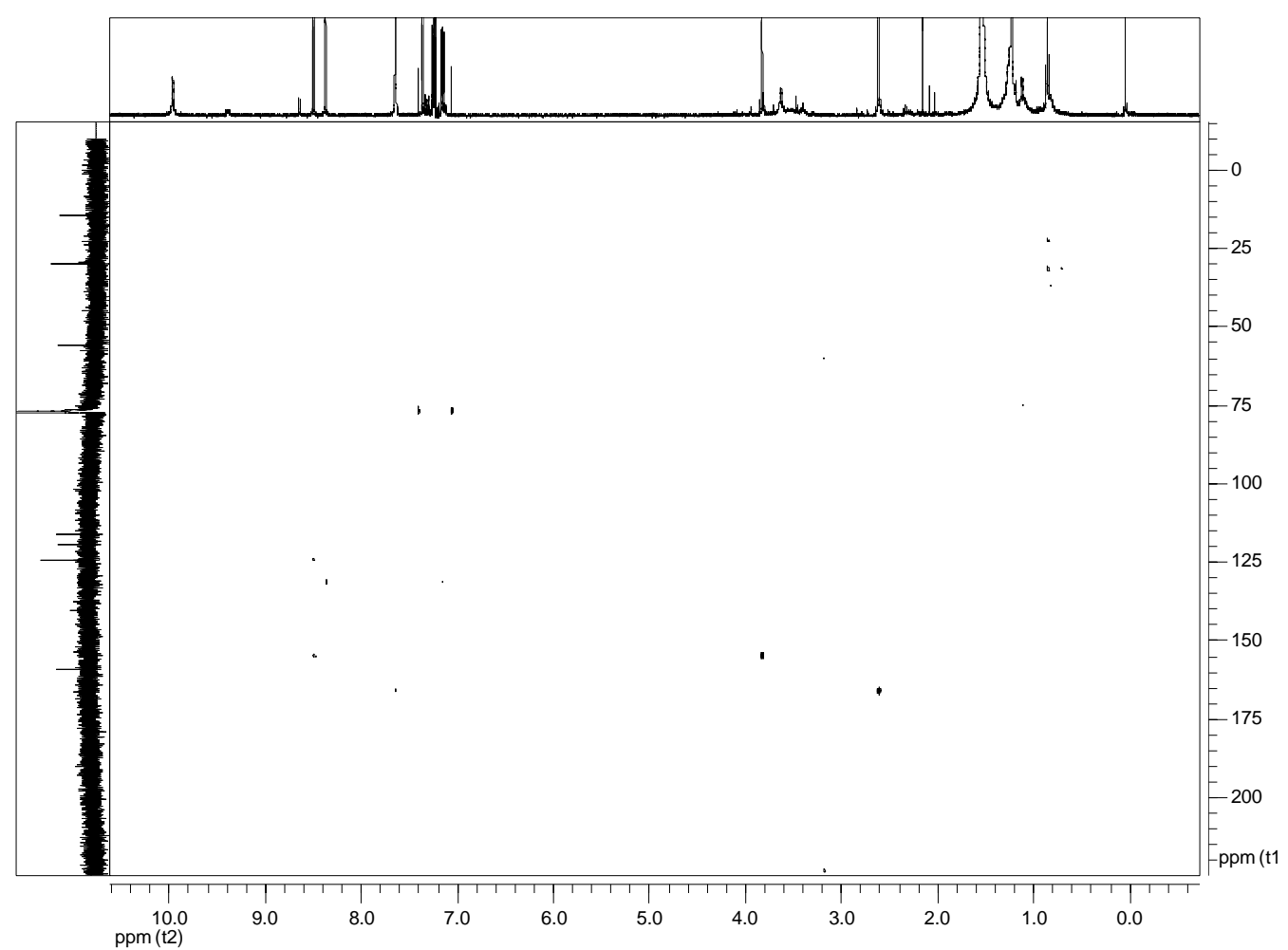

Abb. 47: HMBC-Spektrum ( 150,8 MHz) von Produkt 2a/b in $\mathrm{CD}_{3} \mathrm{OD}$ 


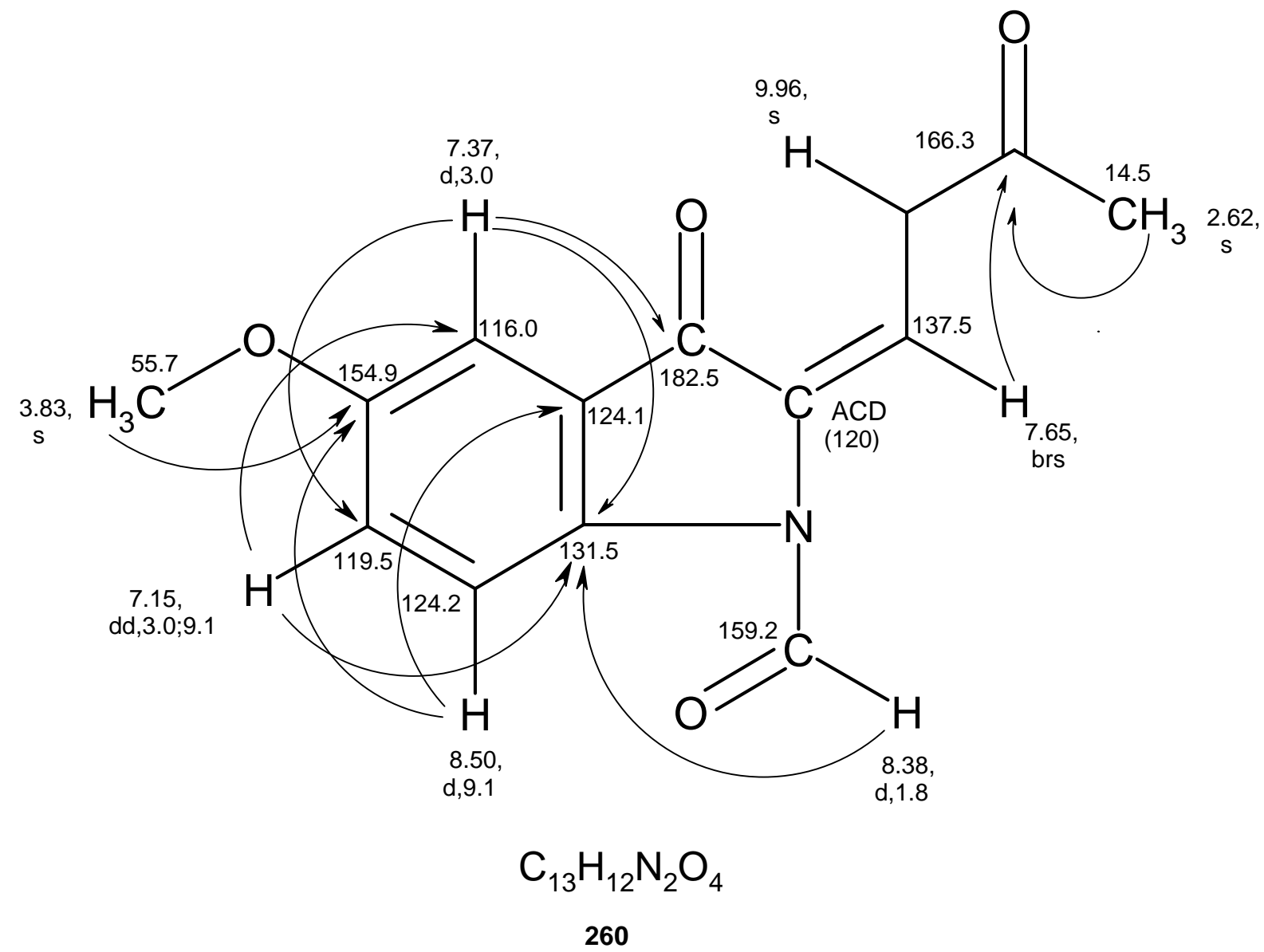

Abb. 48: HMBC-Korrelationen und chemische Verschiebung im H-NMR und C-NMR von Produkt $2 b$.

Zur besseren Übersicht wurde die HBMC-Korrelation an diese Stelle direkt nach dem HMBC-Spektrum (Abb. 47) dargestellt. 


\begin{tabular}{|c|c|c|c|c|}
\hline No. & $\delta_{\mathrm{C}}$ & $\delta_{\mathrm{H}}$ & mult., $J[\mathrm{~Hz}]$ & HMBC \\
\hline За & 124.1 & - & - & - \\
\hline $7 a$ & 131.5 & - & - & - \\
\hline 7 & 124.2 & 8.50 & d, 9.1 & За,5 \\
\hline 6 & 119.5 & 7.15 & dd, 3.0, 9.1 & $7 a$ \\
\hline 5 & 154.9 & - & - & - \\
\hline 4 & 116.0 & 7.37 & d, 3.0 & $3,6,7 a$ \\
\hline $1^{\prime}$ & 137.5 & 7.65 & brs & $1^{\prime \prime}$ \\
\hline 2 & $(120)$ & - & - & - \\
\hline 3 & 182.5 & - & - & - \\
\hline 2', & 14.5 & 2.62 & s & 1 ,' \\
\hline $1^{\prime \prime}$ & 166.3 & - & - & - \\
\hline $5 a$ & 55.7 & 3.83 & $\mathrm{~s}$ & 5 \\
\hline $1 \mathrm{a}$ & 159.2 & 8.38 & $\mathrm{~d}, 1.8$ & $7 a$ \\
\hline
\end{tabular}

Tab. 11: ${ }^{1} \mathrm{H}-\mathrm{NMR}\left(599.7 \mathrm{MHz}, \mathrm{CDCl}_{3}\right)$ und ${ }^{13} \mathrm{C}-\mathrm{NMR}\left(150.8 \mathrm{MHz}, \mathrm{CDCl}_{3}\right)$ Daten von Produkt 2

Die Auswertung aller Spektren ergab schließlich, dass es sich bei der Substanz 2 um zwei Stereoisomere (E,Z-Isomere) handelt muss. Die HMBC-Korrelation für 2b ist in Abb. 48 dargestellt. Die anzunehmenden E,Z-Isomere sind Abb. 49 wiedergegeben<smiles>COc1ccc2c(c1)C(=O)/C(=C/NC(C)=O)N2C=O</smiles>

Produkt 2a<smiles>[2H]N(/C=C1\C(=O)c2cc(OC)ccc2N1C=O)C(C)=O</smiles>

Produkt 2b

Abb. 49: Nummerierungssystem von Produkt 2a/b und dessen mögliche E,Z-Isomere 
Formalname: $N$-(1-Formyl-5-methoxy-3-oxo-1,3-dihydro-indol-2-ylidenemethyl)acetamid

Aussehen: $\quad$ gelblich

UV: $\quad 2 \mathrm{a}: \lambda_{\max } 242$ und $338 \mathrm{~nm}, 2 \mathrm{~b}: \lambda_{\max } 245$ und $384 \mathrm{~nm}$ in EtOH

Fluoreszenz: bei $366 \mathrm{~nm}$ grünlich

Rf-Wert $\quad 0,59$ in Ethylacetat / MetOH $(9.5: 0.5)$

Masse $\quad 260$

Molekülformel: $\mathrm{C}_{13} \mathrm{H}_{12} \mathrm{~N}_{2} \mathrm{O}_{4}$

${ }^{1} \mathbf{H}-\mathbf{N M R}\left(\mathrm{CDCl}_{3}, 599.7 \mathrm{MHz}\right): \delta_{\mathrm{H}}=9.96$ (brs, $\left.1 \mathrm{H}, \mathrm{N}-\mathrm{H}\right), 8.50$ (d, $\left.1 \mathrm{H}, J=9.11,=\mathrm{C}-\mathrm{H}\right), 8.38$ (d, $1 \mathrm{H}, J=1.78 \mathrm{~Hz}, \mathrm{CHO}$ ), 7.65 (brs, $1 \mathrm{H},=\mathrm{CH}-\mathrm{NH}$ ), 7.37 (d, $1 \mathrm{H}, J=2.95$, =C-H), 7.15 (dd, $1 \mathrm{H}, J=2.95$ u. 9.05, =C-H), 3.83 (s, $3 \mathrm{H}, \mathrm{OMe}$ ), 2.62 (s, $3 \mathrm{H}, \mathrm{O}=\mathrm{C}-\mathrm{CH}_{3}$ ).

${ }^{13}$ C-NMR $\left(\mathrm{CDCl}_{3}, 150.8 \mathrm{MHz}\right): \delta_{\mathrm{c}}=182.5\left(\mathrm{C}_{\mathrm{q}}, \mathrm{C}=\mathrm{O}, \mathrm{C}-3\right), 166.3\left(\mathrm{C}_{\mathrm{q}}, \mathrm{O}=\mathrm{C}-\mathrm{CH}_{3}, \mathrm{C}-1\right.$ '’), 159.2 (C $\left.\mathrm{q}_{\mathrm{q}}, \mathrm{CHO}\right), 154.9$ (C $\left.\mathrm{C}_{\mathrm{q}}, \mathrm{C}-5\right), 137.5$ (C $\left.\mathrm{C}_{\mathrm{q}}, \mathrm{C}-1^{\prime}\right), 131.5$ (C, $\left.\mathrm{C}_{\mathrm{q}}, \mathrm{C}-7 \mathrm{a}\right), 124.2$ (C-7), 124.1 (C3a), 119.5 (C-6), 116.0 (C-4), 55.7 (OMe), 14.5 ( $\mathrm{CH}_{3}, \mathrm{C}-2$ '’).

Das Signal für (C-2) war nicht sichtbar, es wurde ein Wert von $\delta_{\mathrm{c}}=120$ angenommen.

(+)-ESI-HRMS: $m / z=261,08705[\mathrm{M}+\mathrm{H}]^{+}$, (calcd. 261,08698 für $\mathrm{C}_{13} \mathrm{H}_{13} \mathrm{~N}_{2} \mathrm{O}_{4}$ ).

(+)-EI-MS: m/z (\%) 260.1 (100). 232.1 (93), 217.12 (7), 191.1 (83), 162.0 (10), 149.1 (16), 134.0 (17), 122.1 (10), 106.0 (7), 95.0 (3), 82.0 (23), 59.0 (4), 54.0 (32), 43.0 (6), 


\subsubsection{Produkt 3}

Die Analyse in der Massenspektroskopie ergab eine Masse von 278. Die durchgeführte HPLC-MS zeigte bei der Retentionszeit von 8.53 eine Pseudomasse $[\mathrm{M}+\mathrm{H}]$ von 279 (Abb. 50), wobei im Gegensatz zu Verbindung 2 nur ein Produktmaximum auftrat.

Das Absorptionsspektrum ist in der Abb. 52 wiedergegeben.

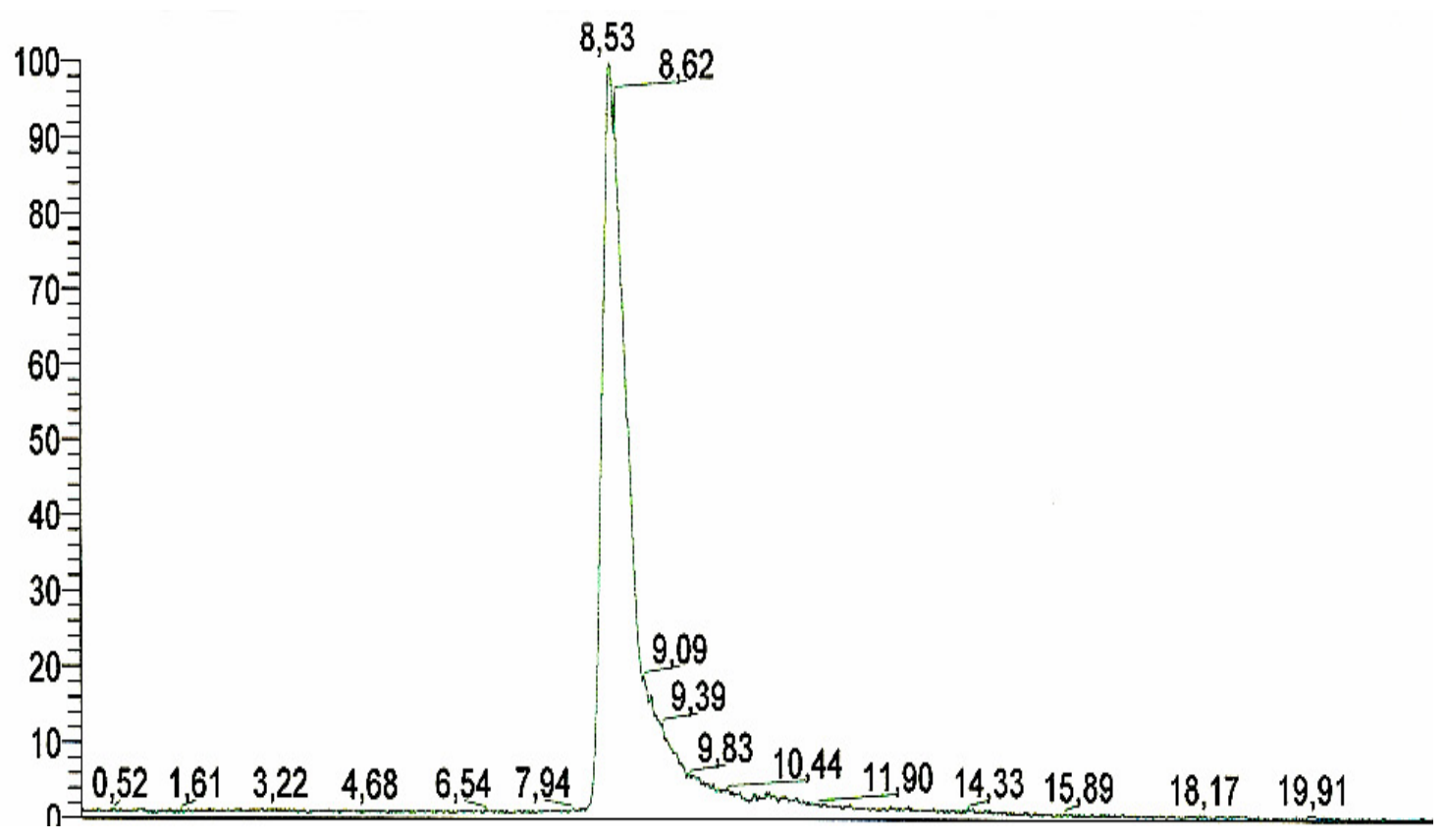

Abb. 50: HPLC-MS (MeOH / $\mathrm{H}_{2} \mathrm{O}, 10-100$ \% MeOH, 0-20 min) von Produkt 3 


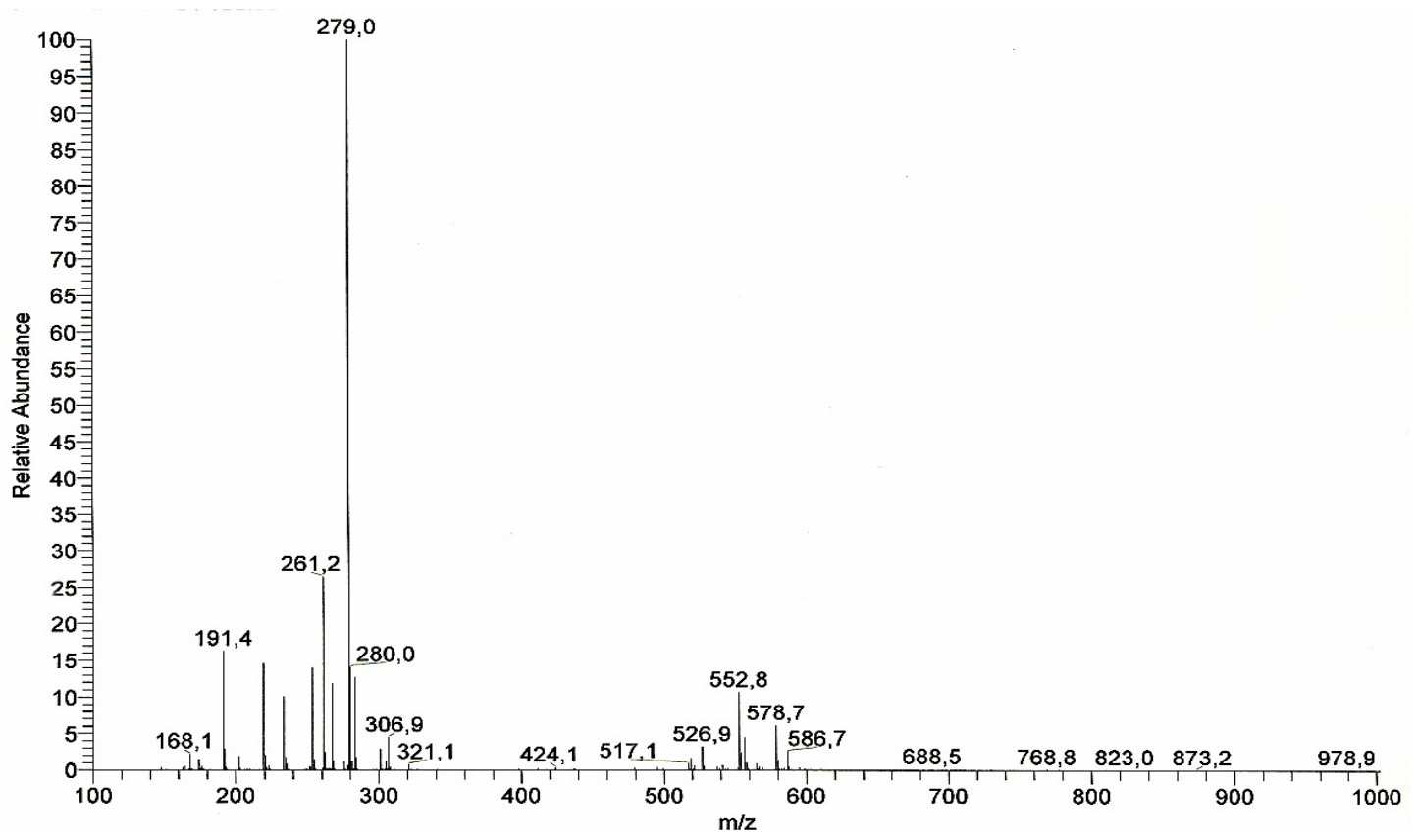

Abb. 51: Massenspektrum (ESI-HRMS, 70 eV) von Produkt 3 (H+)

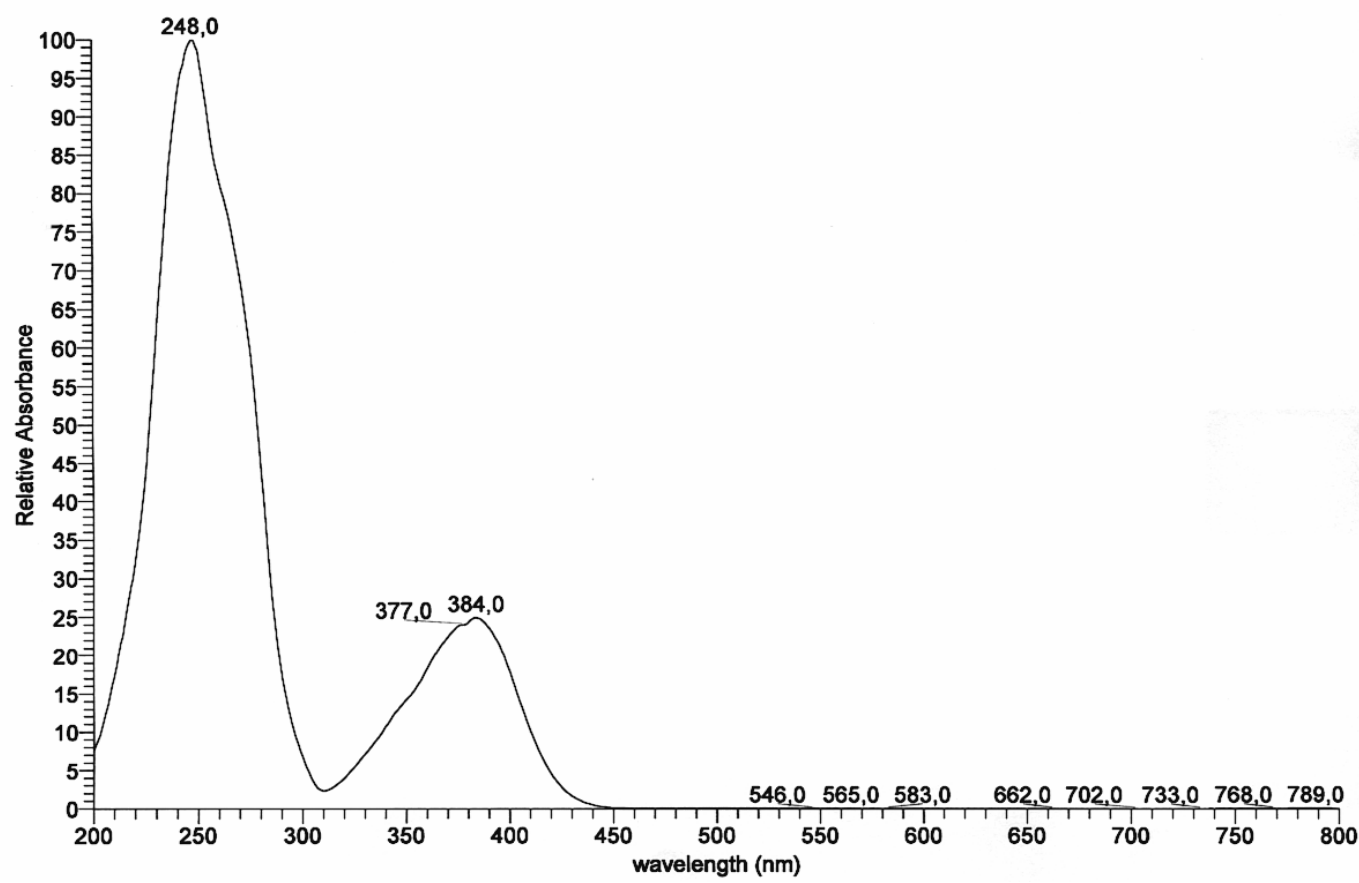

Abb. 52: UV-Spektrum von Produkt 3

Das UV-Spektrum von Produkt 3 (Abb. 52) zeigt bei $\lambda_{\max } 248$ und 384 nm zwei Maxima. Dies sind typische Regionen für ein aromatisches Ringsystem, welches mit Elektronendonatoren substituiert ist. 


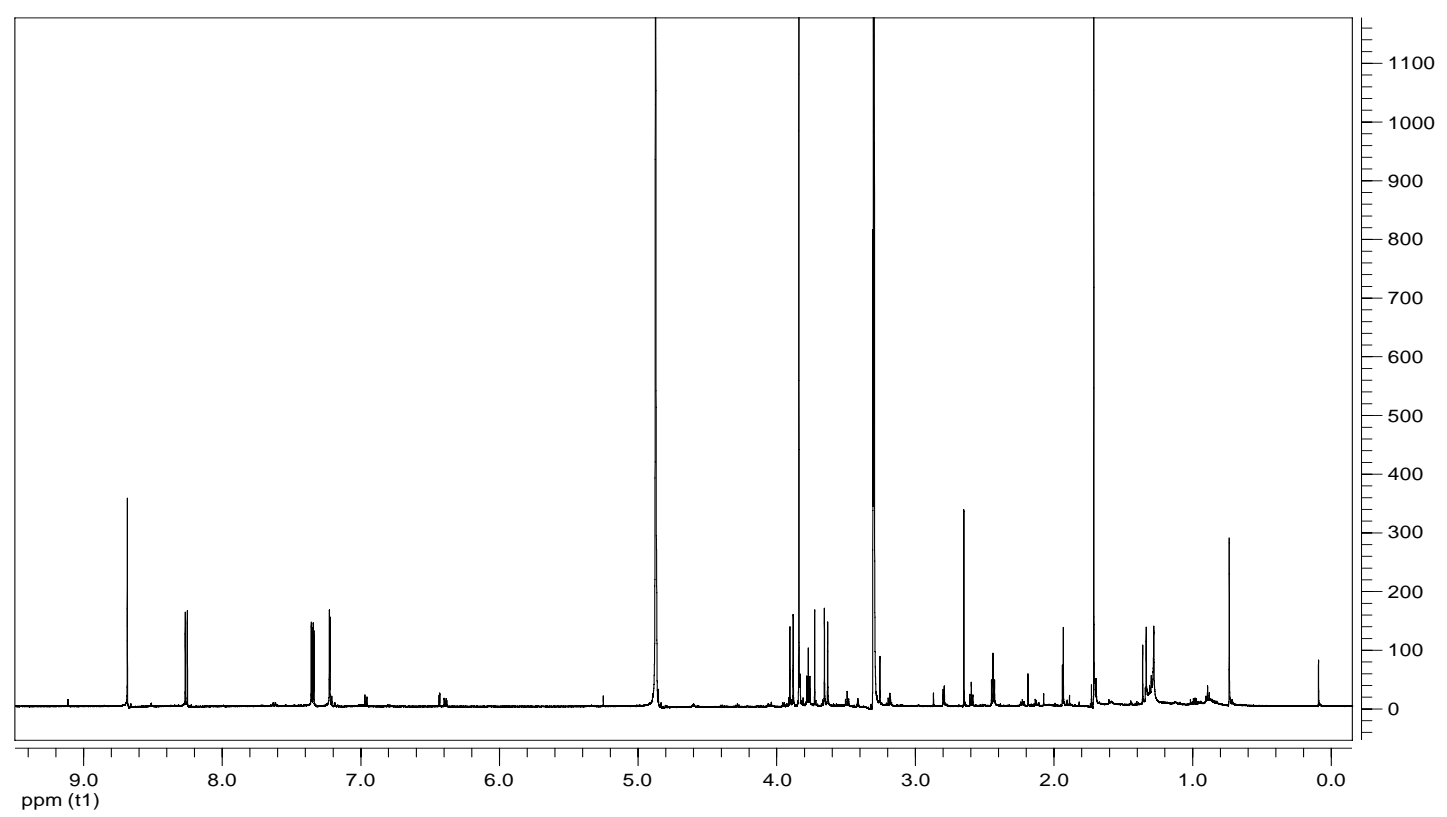

Abb. 53: ${ }^{1} \mathrm{H}-\mathrm{NMR}-S p e k t r u m ~(599,1 \mathrm{MHz})$ von Produkt 3 in $\mathrm{CDCl}_{3}$

Das Singulett bei $\delta_{\mathrm{H}} 3.83$ weist eine Intensität von 3 Protonen auf, die auf eine Methoxygruppe hindeuten. Die für die aromatische Region typischen Signale (Dublette) konnten bei $\delta_{\mathrm{H}}$ 7.22, 7.35 und 8.26 festgestellt werden. Durch HBMC-Spektren (Abb. 57) konnten die Protonen den entsprechenden aromatischen Ringen zugeordnet werden.

Des weiteren konnte ein für eine $\mathrm{CH}_{3}$-Gruppe charakteristisches Singulett bei $\delta_{\mathrm{H}} 1,71$ mit der Intensität von drei Protonen festgestellt werden.

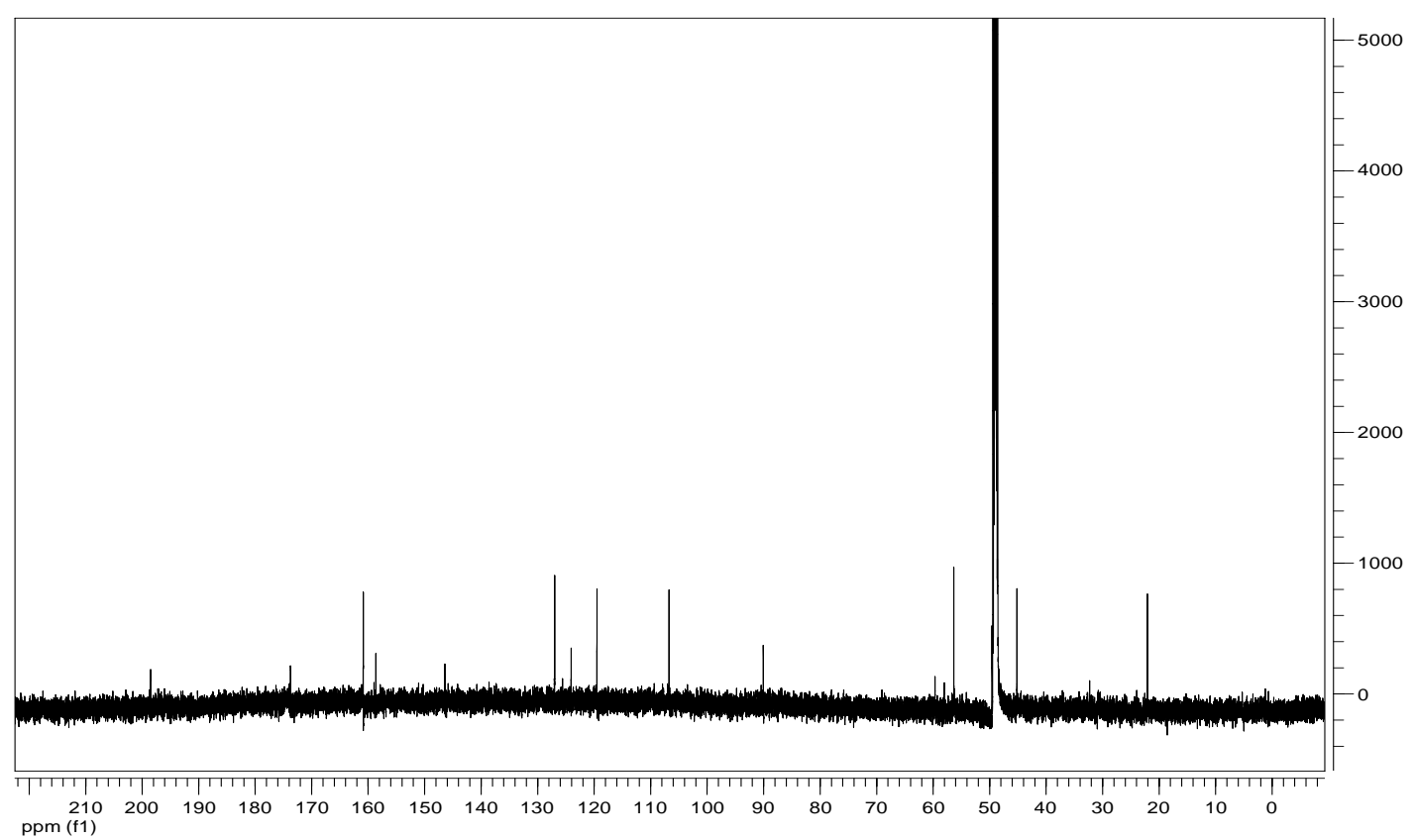

Abb. 54: ${ }^{13} \mathrm{C}-\mathrm{NMR}$ Spektrum (150,8 Mhz) von Produkt 3 in $\mathrm{CD}_{3} \mathrm{OD}$ 
Das C-Spektrum $\left(\mathrm{CD}_{3} \mathrm{OD}\right)$ von Produkt 3 zeigt 13 Kohlenstoff-Signale. Fünf quaternäre Signale gehören zu Carbonylgruppen. In der aliphatischen Region sind ein Methyl-Signal bei $\delta_{\mathrm{C}} 56.3$ und ein Methylen-Signal bei $\delta_{\mathrm{C}} 22.1$ zu erkennen.

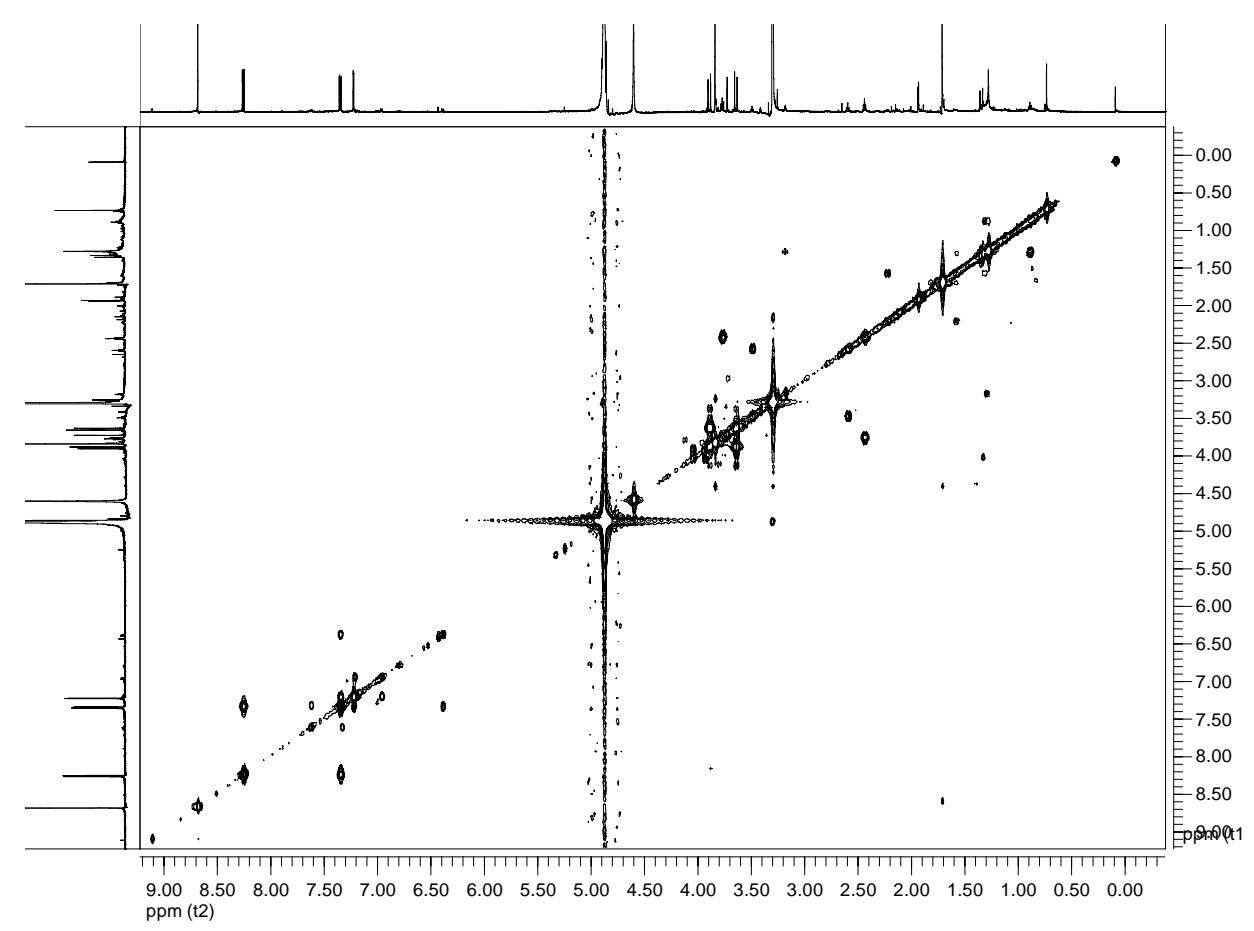

Abb. 55: H,H-COSY-Spektrum (599,1 MHz) von Produkt 3 in $\mathrm{CDCl}_{3}$

Das H,H-COSY-Spektrum (Abb. 55) ergab folgende Strukturfragment:

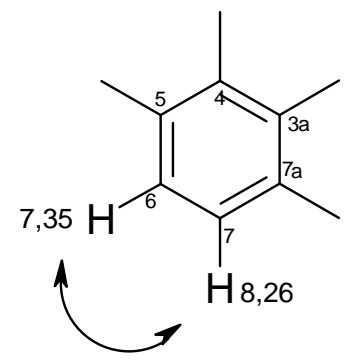

Abb. 56: H,H-Korrelation und chemische Verschiebung im ${ }^{1}$ H-NMR von Produkt 3 


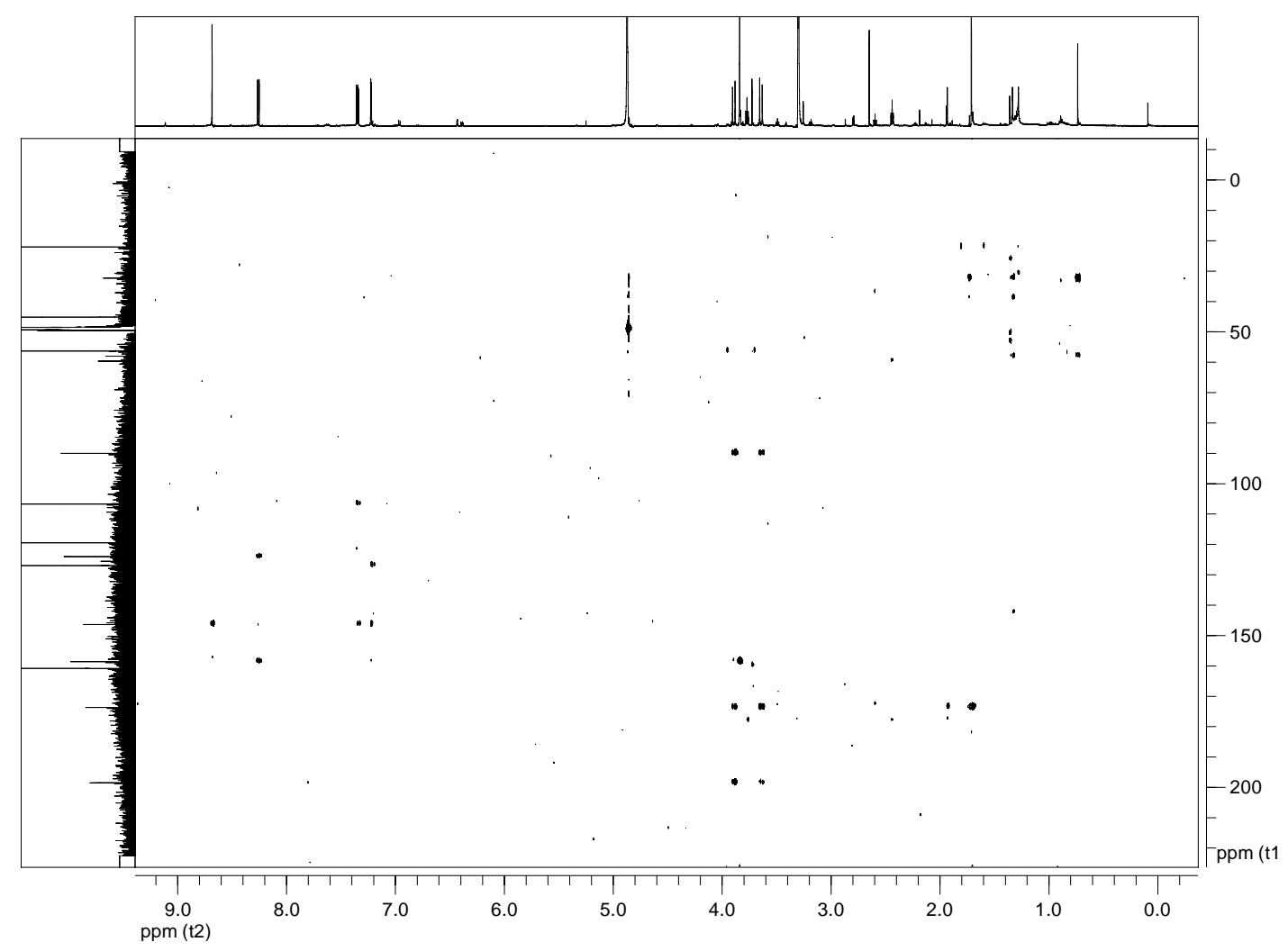

\section{Abb. 57: HMBC-Spektrum (150,8 Mhz) von Produkt 3 in $\mathrm{CD}_{3} \mathrm{OD}$}

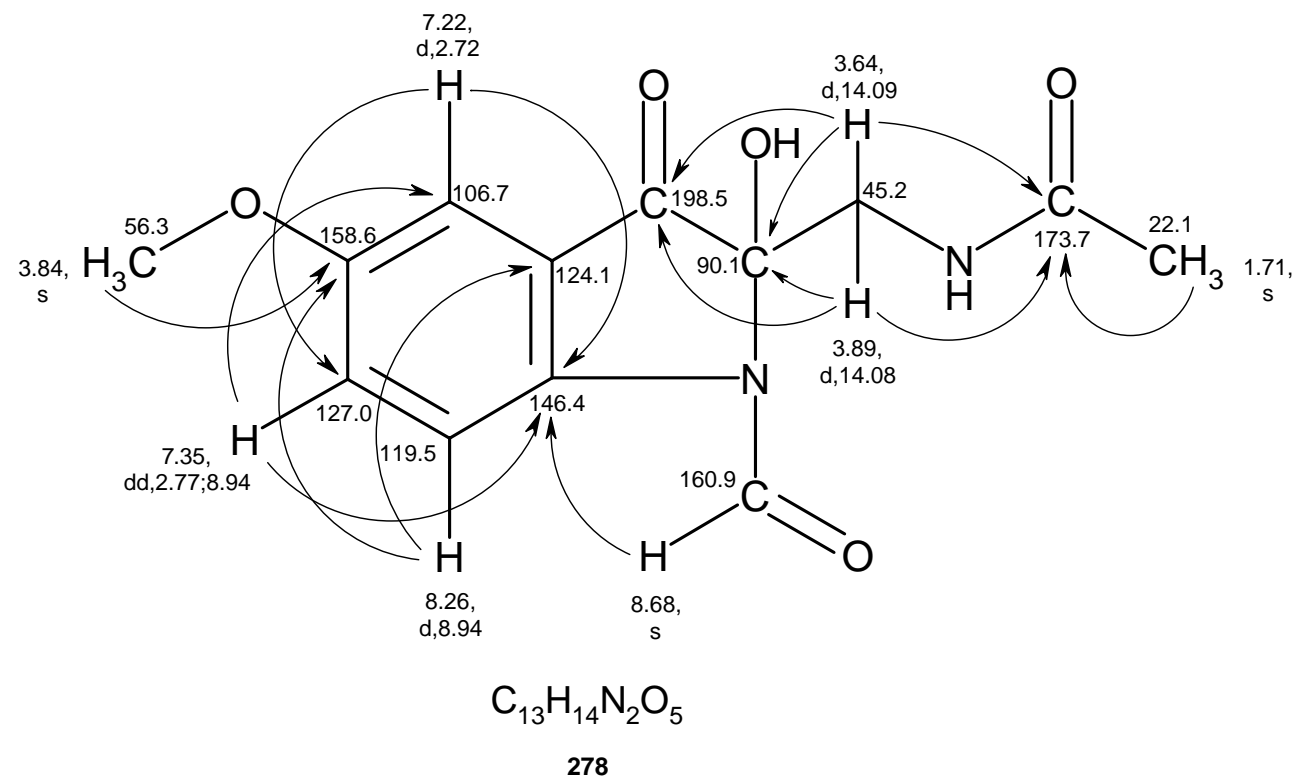

Abb. 58: HMBC-Korrelationen und chemische Verschiebungen im H-NMR und C-NMR Spektren von Produkt 3

Zum besseren Vergleich wurde die HMBC-Korrelation an diese Stelle direkt nach dem HMBC-Spektrum plaziert. 


\begin{tabular}{|c|c|c|c|c|c|c|}
\hline No. & $\begin{array}{c}\delta_{\mathrm{C}} \\
\left(\mathrm{CD}_{3} \mathrm{OD}\right)\end{array}$ & $\begin{array}{c}\delta_{\mathrm{H}} \\
\left(\mathrm{CD}_{3} \mathrm{OD}\right)\end{array}$ & mult., $J[\mathrm{~Hz}]$ & $\begin{array}{c}\delta_{\mathrm{H}} \\
\left(\mathrm{CDCl}_{3}\right)\end{array}$ & mult., $J$ [Hz] & HMBC \\
\hline $3 a$ & 124.1 & - & - & - & - & - \\
\hline $7 a$ & 146.4 & - & - & - & - & - \\
\hline 7 & 119.5 & 8.26 & d, 8.9 & 8.27 & d, 9.0 & $3 a, 5$ \\
\hline 6 & 127.0 & 7.35 & dd, 2.8, 8.9 & 7.27 & dd, 2.8, 9.0 & $4,7 a$ \\
\hline 5 & 158.6 & - & - & & & - \\
\hline 4 & 106.7 & 7.22 & d, 2.7 & 7.14 & d, 2.7 & $6,7 a$ \\
\hline $1^{\prime}$ & 45.2 & $\begin{array}{l}3.64, \\
3.89\end{array}$ & $\mathrm{~d}_{\mathrm{AB}}, 14.1$ & $\begin{array}{l}3.73, \\
3.67\end{array}$ & $\begin{array}{c}\text { dd, 14.6, } \\
\text { dd,16.1 }\end{array}$ & $2,3,1^{\prime \prime}$ \\
\hline 2 & 90.1 & - & - & & & - \\
\hline 3 & 198.5 & - & - & & & - \\
\hline $2^{\prime \prime}$ & 22.1 & 1.71 & $\mathrm{~s}$ & 2.08 & $\mathrm{~s}$ & 1'” \\
\hline 1'” & 173.7 & - & - & & & - \\
\hline $5 a$ & 56.3 & 3.83 & $\mathrm{~s}$ & 3.81 & $\mathrm{~s}$ & 5 \\
\hline $1 \mathrm{a}$ & 160.9 & 8.68 & $\mathrm{~s}$ & 8.71 & $\mathrm{~S}$ & $7 a$ \\
\hline
\end{tabular}

Tab. 12: ${ }^{1} \mathrm{H}-\mathrm{NMR}$ (599.7 MHz, CD $\left.3 \mathrm{OD}\right)$ und ${ }^{13} \mathrm{C}-\mathrm{NMR}$ (150.8 MHz, CD $\left.3 \mathrm{OD}\right)$ ${ }^{1}$ H-NMR (599.7 MHz, CDCl 3 ) Daten von Produkt 3

Die Suche nach Publikationen in der Datenbank der „Chemical Abstracts“ blieb ohne Ergebnis, so dass davon ausgegangen werden muss, dass es sich hierbei um eine neue Substanz handelt.

Die Ermittlung des DBE-Wertes von der Strukturformel von Produkt 3 beträgt 8. Dieser Wert stimmt mit der gefundenen Molekülformel $\mathrm{C}_{13} \mathrm{H}_{14} \mathrm{~N}_{2} \mathrm{O}_{5}$ der Substanz überein.

Nach Auswertung aller Spektren ergab sich schließlich folgende Struktur:<smiles>COc1ccc2c(c1)C(=O)C(O)(CNC(C)=O)N2C=O</smiles>

Abb. 60: Nummerierungssystem von Produkt 3: 
Formalname: $N$-(1-Formyl-2-hydroxy-5-methoxy-3-oxo-2,3-dihydro-1H-indol-2ylmethyl)-acetamid

Aussehen: gelblich

UV: $\quad \lambda_{\max } 248$ und $384 \mathrm{~nm}$ in EtOH

Fluoreszenz: $\quad \mathrm{Ex}=378, \mathrm{Em}=484 \mathrm{~nm}$ (bei $366 \mathrm{~nm}$ bläulich)

Rf-Wert: $\quad 0,41$ in Ethylacetat / MetOH (9.5: 0.5)

Masse: $\quad 278$

Molekülformel: $\mathrm{C}_{13} \mathrm{H}_{14} \mathrm{~N}_{2} \mathrm{O}_{5}$

${ }^{1} \mathbf{H}-\mathbf{N M R}\left(\mathrm{CD}_{3} \mathrm{OD}, 599.7 \mathrm{MHz}\right): \delta_{\mathrm{H}}=8.68(\mathrm{~s}, 1 \mathrm{H}, \mathrm{CHO}), 8.26(\mathrm{~d}, J=8.9 \mathrm{~Hz}, 1 \mathrm{H},=\mathrm{C}-\mathrm{H})$, 7.35 (dd, $J=8.9$ u. $2.8 \mathrm{~Hz}, 1 \mathrm{H},=\mathrm{C}-\mathrm{H}), 7.22$ (d, $J=2.7 \mathrm{~Hz}, 1 \mathrm{H},=\mathrm{C}-\mathrm{H}), 3.89,3.64\left(\mathrm{~d}_{\mathrm{AB}}, J=\right.$ $14.1 \mathrm{~Hz}, 2 \mathrm{H}, \mathrm{CH}_{2}-\mathrm{NH}$ ), 3.84 (s, $3 \mathrm{H}, \mathrm{OMe}$ ), 1.71 (s, $3 \mathrm{H}, \mathrm{O}=\mathrm{C}-\mathrm{CH}_{3}$ ).

${ }^{1} \mathbf{H}-\mathrm{NMR}\left(\mathrm{CDCl}_{3}, 599.7 \mathrm{MHz}\right): \delta_{\mathrm{H}}=8.71$ (s, $\left.1 \mathrm{H}, \mathrm{CHO}\right), 8.27(\mathrm{~d}, J=9.0,1 \mathrm{H},=\mathrm{C}-\mathrm{H}), 7.27$ (dd, $J=9.0$ u. $2.9 \mathrm{~Hz}, 1 \mathrm{H}$, =C-H), 7.14 (d, $J=2.65 \mathrm{~Hz}, 1 \mathrm{H},=\mathrm{C}-\mathrm{H}$ ), 6.17 (brs, $1 \mathrm{H}, \mathrm{N}-\mathrm{H}$ ), 3.81 (s, 3 H, OMe), 3.73 (dd, 1 H, $J=6.94$ u. 16.16 Hz, $\mathrm{CH}_{2}-\mathrm{NH}$ ), 3.67 (dd, $1 \mathrm{H}, J=6.37$ u. $14.68 \mathrm{~Hz}, \mathrm{CH}_{2}-\mathrm{NH}$ ), 2.08 (s, $3 \mathrm{H}, \mathrm{O}=\mathrm{C}-\mathrm{CH}_{3}$ ).

${ }^{13}$ C-NMR (CD $\left.{ }_{3} \mathrm{OD}, 150.8 \mathrm{MHz}\right): \delta_{\mathrm{C}}=198.5\left(\mathrm{C}_{\mathrm{q}}, \mathrm{C}=\mathrm{O}, \mathrm{C}-3\right), 173.7\left(\mathrm{C}_{\mathrm{q}}, \mathrm{O}=\mathrm{C}-\mathrm{CH}_{3}, \mathrm{C}-1\right.$ '’), 160.9 (C $\left.\mathrm{C}_{\mathrm{q}}, \mathrm{CHO}\right), 158.6$ (C $\left.\mathrm{q}_{\mathrm{q}}, \mathrm{C}-5\right), 146.4$ (C $\left.\mathrm{q}, \mathrm{C}-7 \mathrm{a}\right), 127.0$ (C-6), 124.1 (C-3a), 119.5 (C-7), 106.7 (C-4), 90.1 (C-OH, C-2), 56.3 (OMe), 45.2 ( $\mathrm{CH}_{2}-\mathrm{NH}, \mathrm{C}-1$ '), 22.1 ( $\mathrm{CH}_{3}, \mathrm{C}-2$ '’).

(+)-ESI-HRMS: $m / z=279,09765[\mathrm{M}+\mathrm{H}]^{+}$, (calcd. 279,09755 für $\mathrm{C}_{13} \mathrm{H}_{15} \mathrm{~N}_{2} \mathrm{O}_{5}$ ), 301,07975 $[\mathrm{M}+\mathrm{Na}]^{+}$, (calcd. 301,07949 für $\mathrm{C}_{13} \mathrm{H}_{14} \mathrm{~N}_{2} \mathrm{O}_{5} \mathrm{Na}$ ).

(+)-EI-MS: $m / z(\%)=278.1$ (5), 250.2 (6),221.1 (2), 207.1 (25), 179.1 (100),. 178.1 (15), 136.1 (5), 79.0 (4), 72.1 (14), 43.0 (38). 


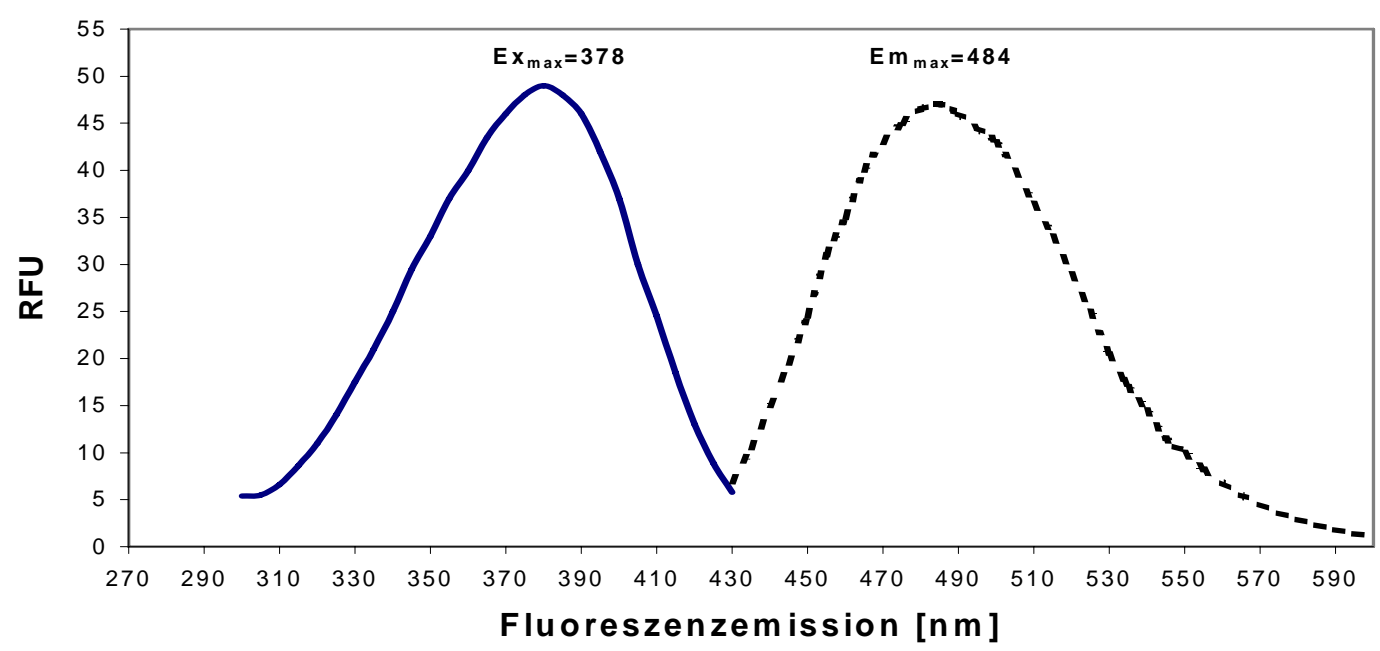

Abb. 59: Excitations- und Emissionsspektrum von Produkt 3

Durchgezogene Linie: Excitationsspektrum, Emissionswellenlänge 484 nm; gestrichelte Linie: Emissionsspektrum, Excitationswellenlänge 378 nm.

\subsubsection{Umsatzanalyse von Produkt 2 und 3}

In einen nachfolgenden Experiment wurde geprüft, welche Abhängigkeiten zwischen Substanz 2 und 3 bestehen. Dazu wurde ein 10-facher Ansatz des ABTS-Kationradikal-Assay (siehe 2.3.3) eingesetzt. Das Reaktionsgemisch wurde gemäß 2.4 Extraktion aufbereitet und auf eine DC aufgetragen. Die Banden der Substanzen 2 und 3 wurden gemäß 2.4 Reelution präpariert und in einem jeweils 1-fachen ABTS-Kationradikal-Assay inkubiert.

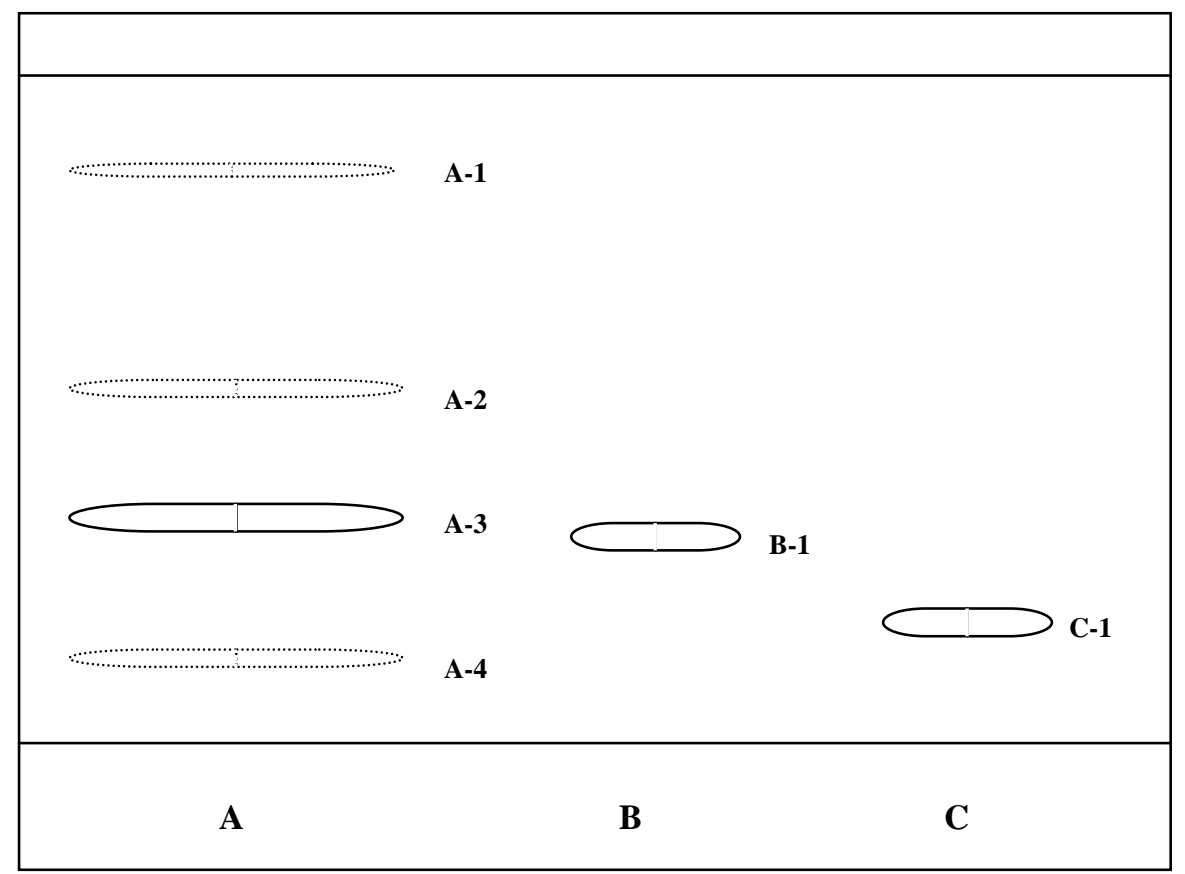

Abb. 61: Umsatz Produkt 2 mit ABTS ${ }^{\bullet+}$ 
Tab. 13: Charakterisierung der Banden aus Abb. 61

\begin{tabular}{|c|l|c|l|c|l|c|}
\hline Bande & \multicolumn{1}{|c|}{ Spur A } & $\begin{array}{c}\text { Rf- } \\
\text { Wert }\end{array}$ & \multicolumn{1}{|c|}{ Spur B } & $\begin{array}{c}\text { Rf- } \\
\text { Wert }\end{array}$ & Spur C & $\begin{array}{c}\text { Rf- } \\
\text { Wert }\end{array}$ \\
\hline 1 & $254(+)$ & 0,89 & Mel & 0,35 & AFMK & 0,21 \\
\hline 2 & $366(+)$ & 0,56 & & & & \\
\hline 3 & Produkt 3 & 0,36 & & & & \\
\hline 4 & $366(+)$ & 0,13 & & & & \\
\hline
\end{tabular}

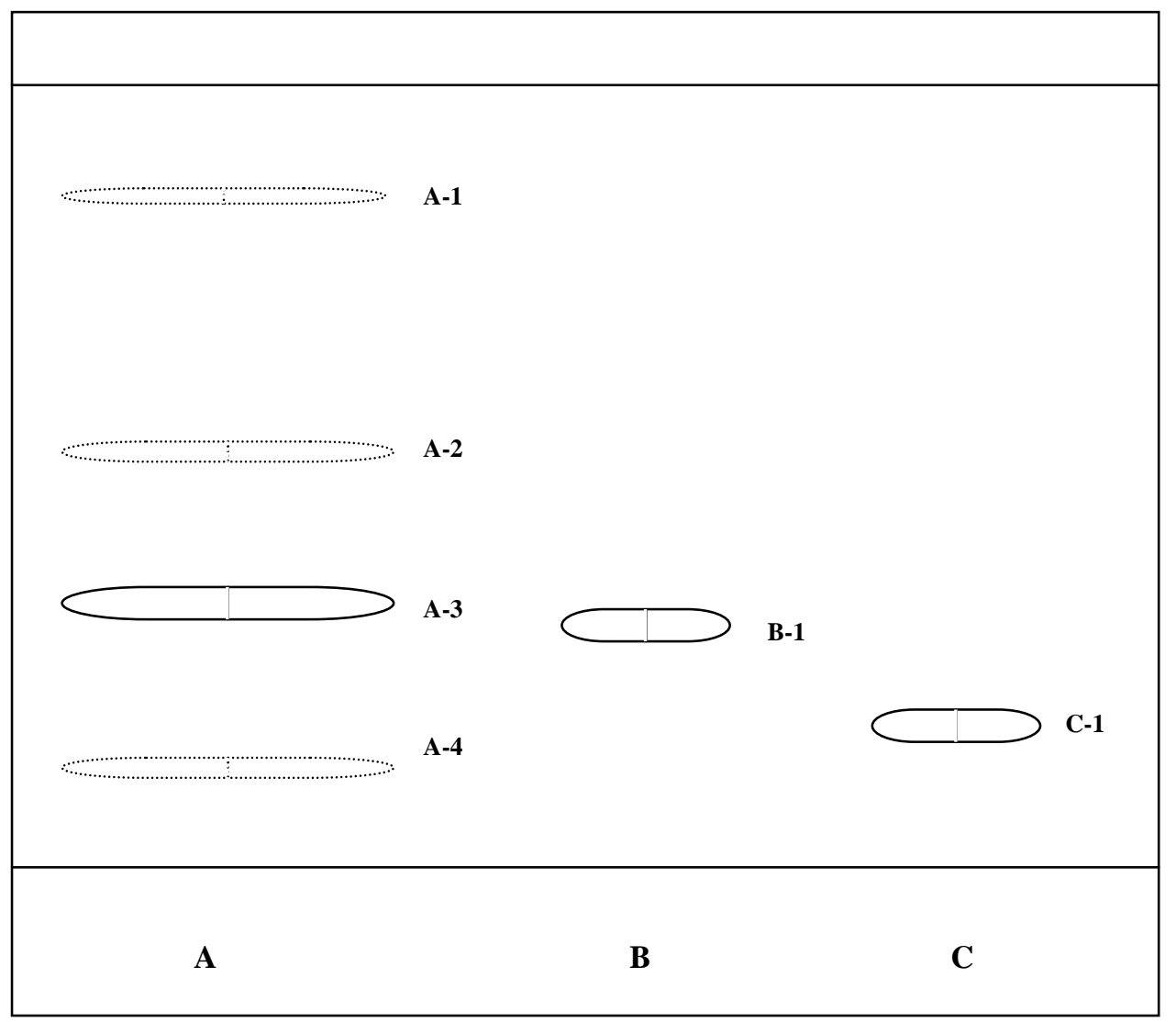

Abb. 62: Umsatz Produkt 3 mit ABTS ${ }^{\bullet+}$ 
Tab. 14: Charakterisierung der Banden aus Abb. 62

\begin{tabular}{|c|l|c|l|c|c|c|}
\hline Bande & \multicolumn{1}{|c|}{ Spur A } & $\begin{array}{c}\text { Rf- } \\
\text { Wert }\end{array}$ & \multicolumn{1}{|c|}{ Spur B } & $\begin{array}{c}\text { Rf- } \\
\text { Wert }\end{array}$ & Spur C & $\begin{array}{c}\text { Rf- } \\
\text { Wert }\end{array}$ \\
\hline 1 & $254(+)$ & 0,85 & Melatonin & 0,30 & AFMK & 0,17 \\
\hline 2 & $366(+)$ & 0,52 & & & & \\
\hline 3 & Produkt 3 & 0,33 & & & & \\
\hline 4 & $366(+)$ & 0,11 & & & & \\
\hline
\end{tabular}

Die Ergebnisse dieser Versuche (Abb. 61, 62) zeigt eindeutig, dass das Produkt 3 aus Produkt 2 (a oder b) hervorgeht, jedoch nicht Produkt 2 a/b durch Wasserabspaltung in Produkt 3 übergehen kann. 


\section{$\underline{\text { 3.4.3 Produkt } 4}$}

Durch eine weitere Aufreinigung der Substanz 2 konnten zwei weitere Produkte isoliert werden. In der Reihenfolge ihres Erscheinens wurde sie mit Produkt 4 und 5 benannt.

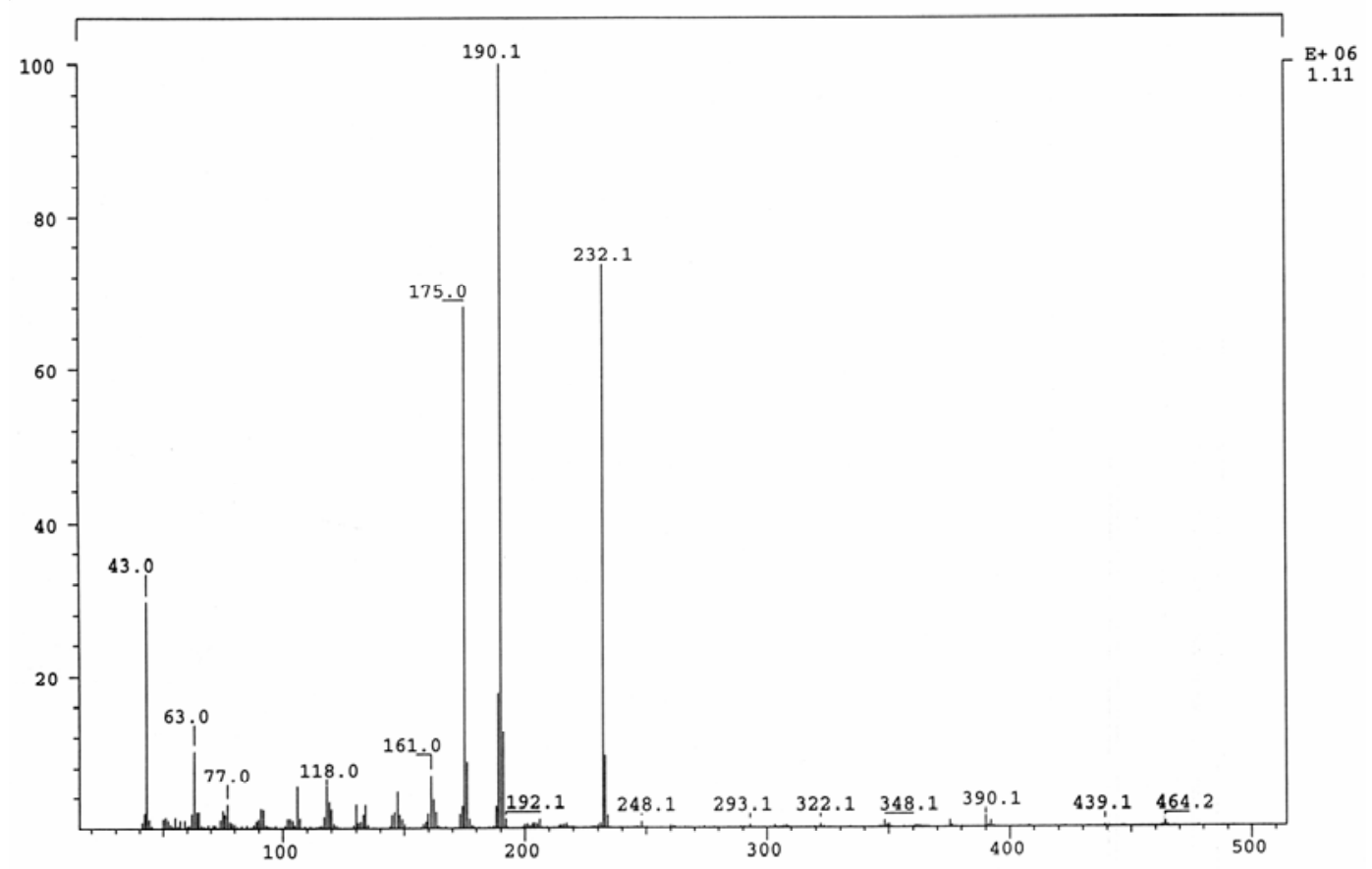

Abb. 63: Massenspektrum (EI-MS, 70 eV) von Produkt 4

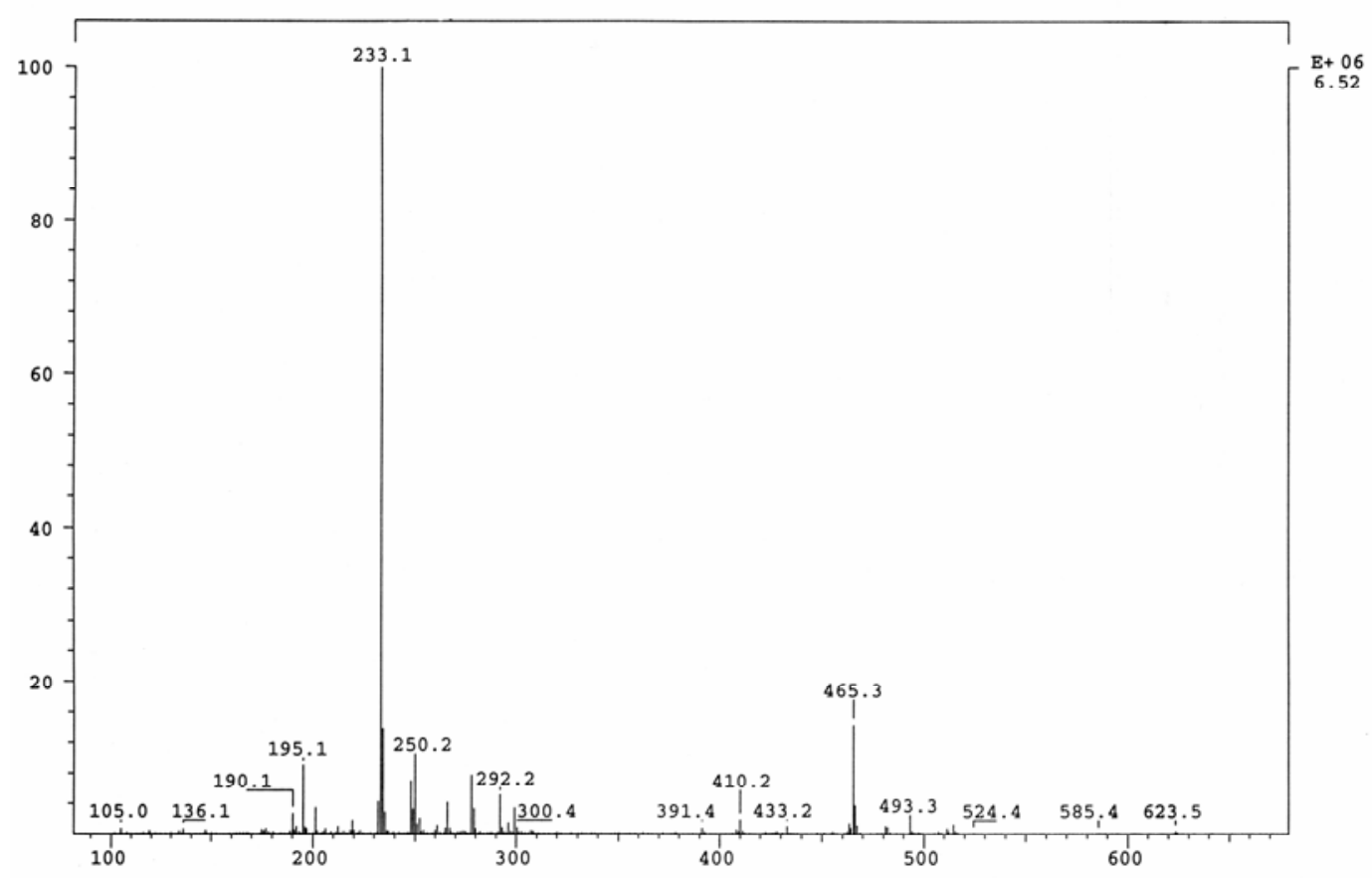

Abb. 64: Massenspektrum (CI-MS, 70 eV) von Produkt 4 (H+) 


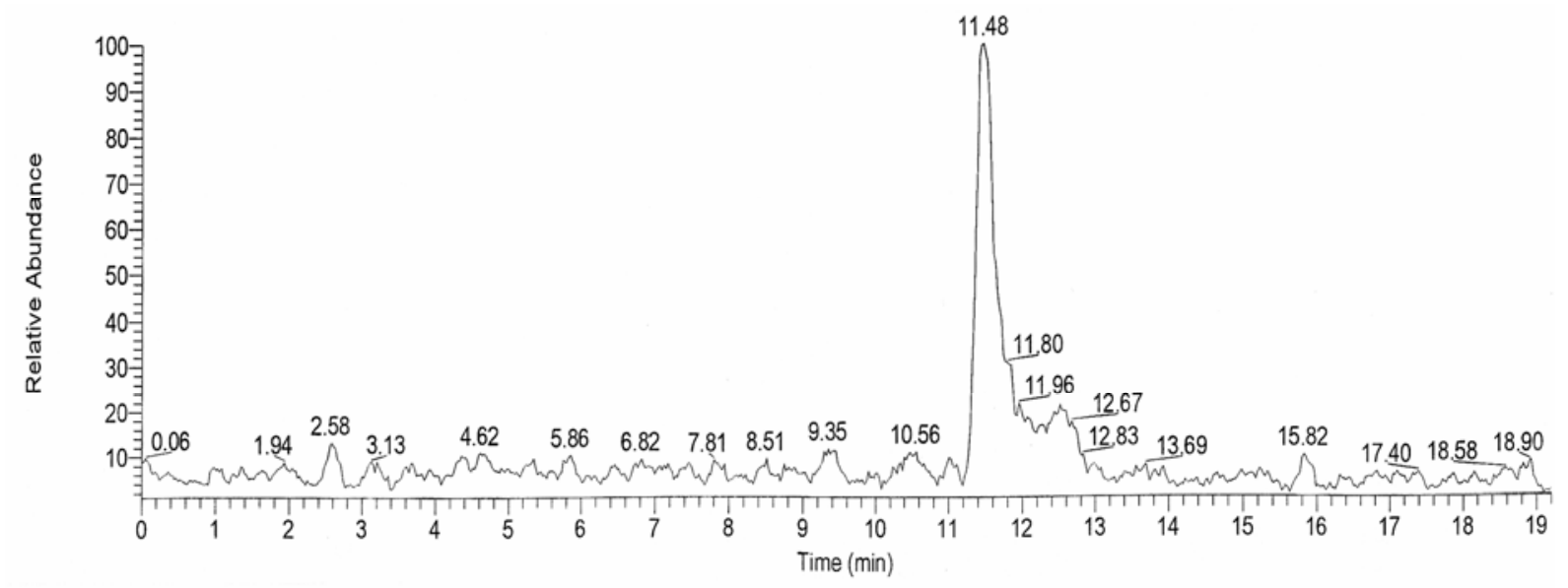

Abb. 65: HPLC-MS (MeOH / $\mathrm{H}_{2} \mathrm{O}, 10-100 \% \mathrm{MeOH}, 0-20$ min) von Produkt 4

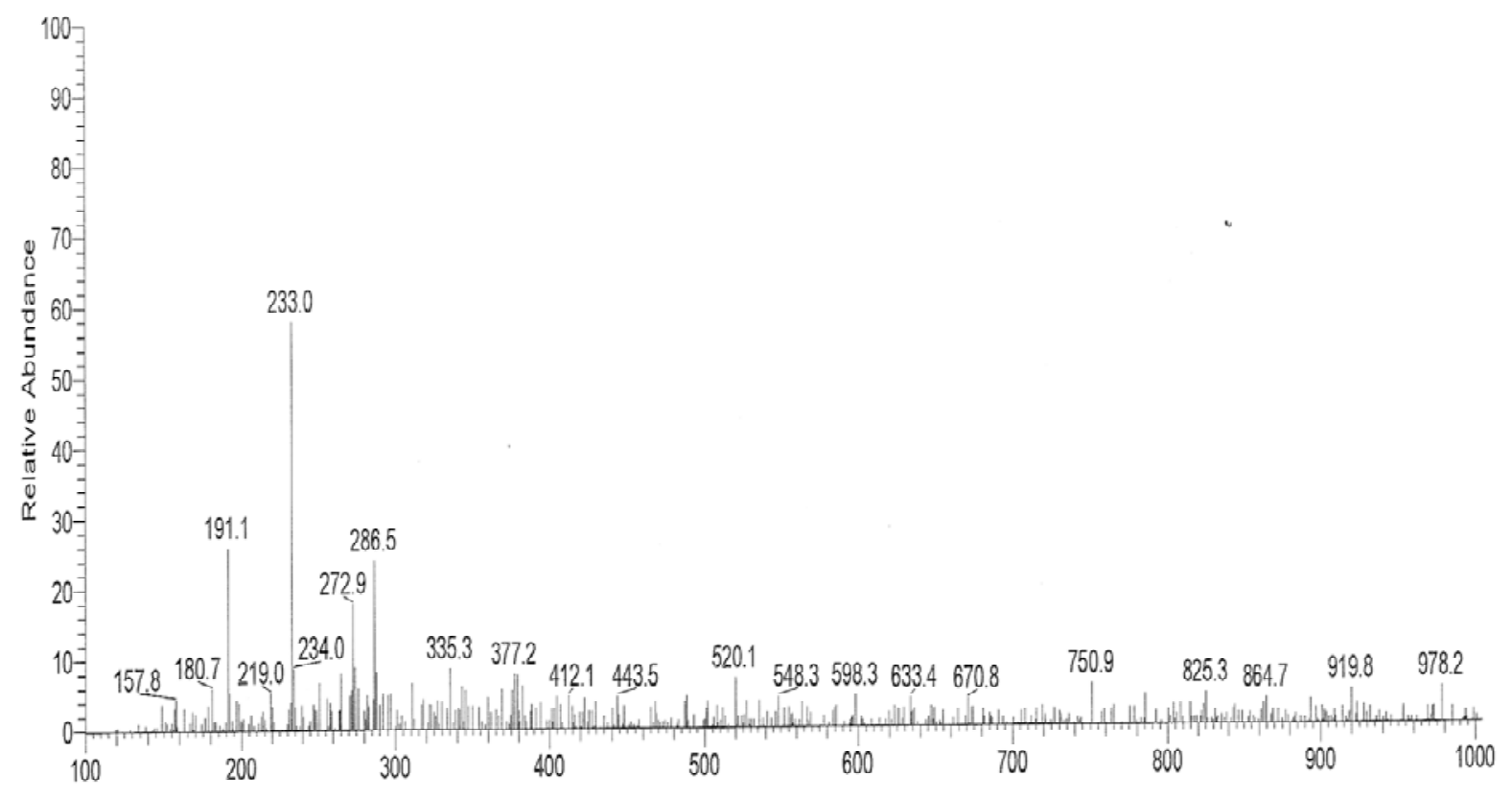

Abb. 66: Massenspektrum (ESI-HRMS, 70 eV) von Produkt 4 (H+) 
Auf Grund der geringen Menge der Substanzen konnte weder ein UV-Spektrum noch ein C-NMR-Spektrum aufgenommen werden.

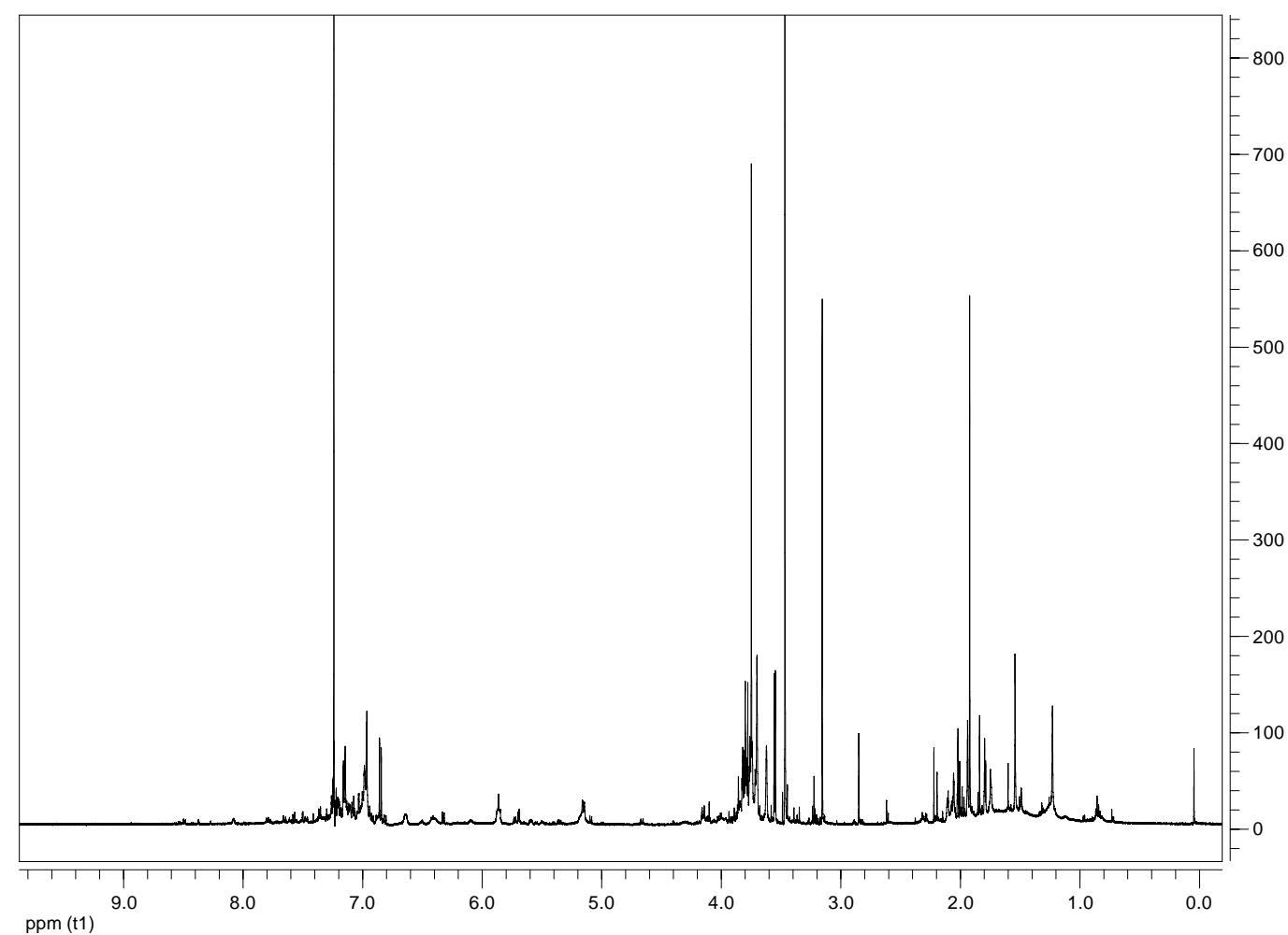

Abb. 67: ${ }^{1} \mathrm{H}-\mathrm{NMR}-S p e k t r u m ~\left(599,1 \mathrm{MHz}\right.$ ) von Produkt 4 in $\mathrm{CDCl}_{3}$

Das H-NMR-Spektrum beinhaltet noch Verunreinigungen, welche aber noch tolerierbar sind, denn eine weitere Aufreinigung von Produkt 4, hätte die Nachweisbarkeitsgrenze der H-NMR unterschritten.

Ein Singulett bei $\delta_{\mathrm{H}} 3.70$ mit der Intensität von 3 Protonen deutet auf eine Methoxygruppe hin. Das Singulett bei $\delta_{\mathrm{H}} 1.92$ mit der Intensität von 3 Protonen weist auf eine $\mathrm{CH}_{3}$-Gruppe hin. Typische Signale für den aromatischen Bereich konnten bei $\delta_{\mathrm{H}}$ 6.85, 6.96 und 7.15 entdeckt werden. 


\begin{tabular}{|c|c|c|c|c|c|c|}
\hline No. & $\delta_{\mathrm{C}-\mathrm{ACD}} \|$ & $\delta_{\mathrm{H}-\mathrm{ACD}}$ & $\delta_{\text {C-ACD }} \|$ & $\delta_{\mathrm{H}-\mathrm{ACD}}$ & $\delta_{\mathrm{H}}$ & mult., $J[\mathrm{~Hz}]$ \\
\hline 1 & $\mathrm{~N}=$ & $=$ & $\mathrm{N} \|$ & $\|$ & & \\
\hline 2 & 122,45 & & 145,76 & & 3,15 & $\mathrm{~s}$ \\
\hline 3 & 185,81 & & 195,54 & & 3,46 & $\mathrm{~s}$ \\
\hline 3a & 119,90 & & 125,45 & & 3,55 & d, 6,28 \\
\hline \multirow[t]{2}{*}{4} & 109,32 & 7,06 & 111,94 & 7,35 & 6,96 & \\
\hline & & $3,2 \mathrm{~d}$ & & $2,36 \mathrm{~d}$ & & d, 2,72 \\
\hline 5 & 152,37 & & 152,03 & & 3,62 & s \\
\hline $5 a$ & 55,60 & $3,77 \mathrm{~s}$ & 55,34 & $3,78 \mathrm{~s}$ & 3,70 & $\mathrm{~s}$ \\
\hline \multirow[t]{2}{*}{6} & 126,57 & 7,12 & 121,41 & 7,05 & 7,15 & \\
\hline & & 3,2/8,6 dd & & 2.36/8,42 dd & & dd, 8,7; 2,6 \\
\hline 7 & 116,02 & $\begin{array}{c}6,71 ; \\
8,60 \text { dd }\end{array}$ & 121,72 & $\begin{array}{c}\text { 7,76; } \\
\text { 8,42 dd }\end{array}$ & 6,85 & d, 8,80 \\
\hline $7 a$ & 146,51 & & 152,58 & & & \\
\hline $1^{\prime}$ & 127,41 & $5,27 / 6,58 \mathrm{~s}$ & 32,40 & $4,83 \mathrm{~s}$ & 3,75 & $\mathrm{~s}$ \\
\hline $2^{\prime}$ & $\mathrm{NH}$ & & $\mathrm{NH}$ & & 4,15 & d, 10,80 \\
\hline 1 '” & 164,88 & & 170,30 & & 5,15 & d, 9,18 \\
\hline $2 ’$ & 23,26 & $1,92 \mathrm{~s}$ & 22,80 & $1,91 \mathrm{~s}$ & 1,92 & $\mathrm{~s}$ \\
\hline
\end{tabular}

Tab. 15: ${ }^{1} \mathrm{H}-\mathrm{NMR}$ (599.7 MHz, CD $\left.3 \mathrm{OD}\right)$ und ${ }^{13} \mathrm{C}-\mathrm{NMR}(150.8 \mathrm{MHz})$ Daten von Produkt 4

Zur Ermittlung einer chemischen Struktur wurden die Daten aus der H-NMR mit berechneten Daten des Chemie Programms ACD ergänzt und miteinander verglichen.

Unter anderem wurden dabei zwei Strukturvarianten betrachtet.

$\mathrm{N} \| \rightarrow$ Doppelbindung im neu gebildeten hetrozyklischen Ring, keine in der Seitenkette.

$\mathrm{N}=\rightarrow$ Doppelbindung in der Seitenkette, dafür keine im hetrozyklischen Ring. 


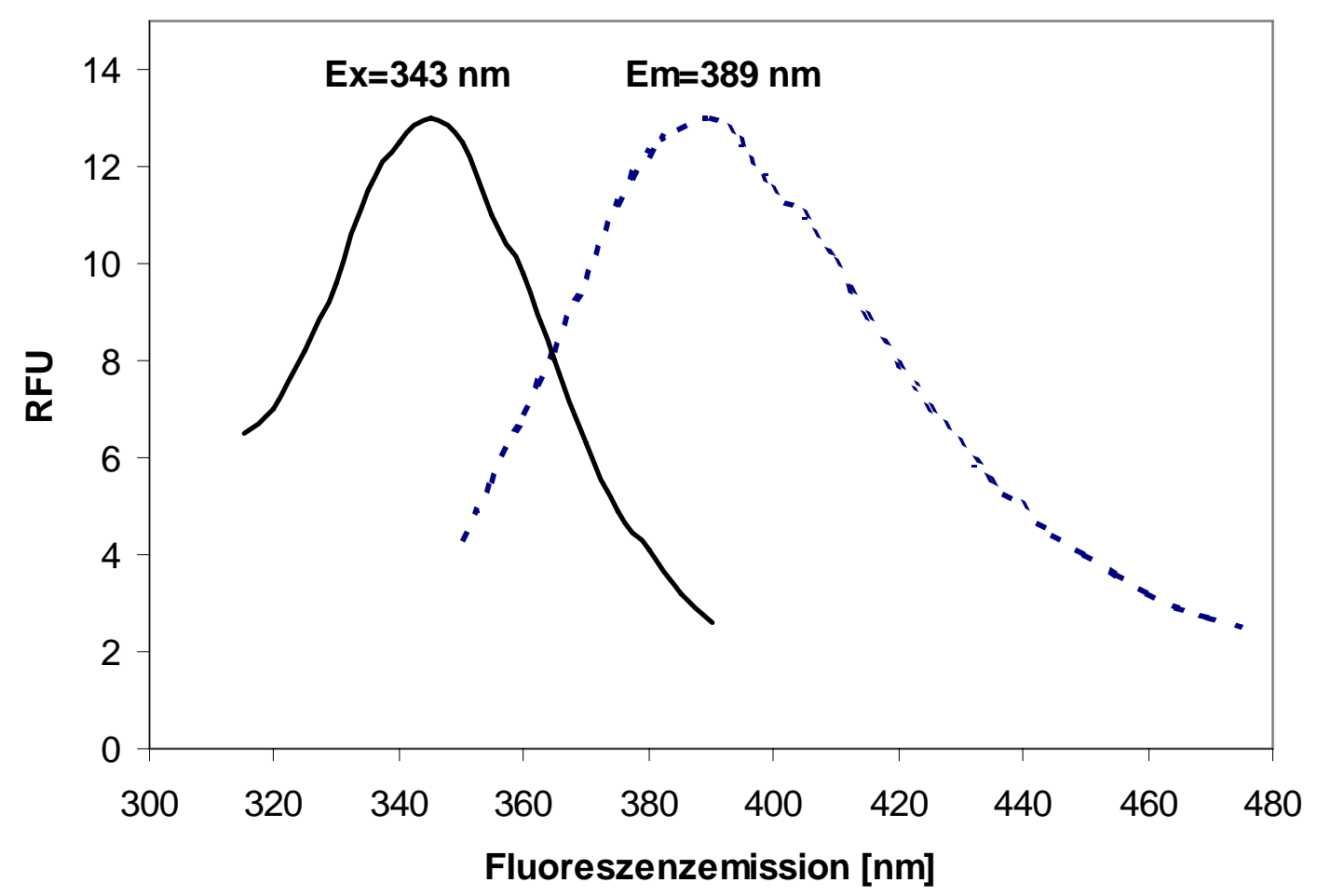

Abb. 68: Excitations- und Emissionsspektrum von Produkt 4

Durchgezogene Linie: Excitationsspektrum, Emissionswellenlänge 389 nm; gestrichelte Linie: Emissionsspektrum, Excitationswellenlänge $343 \mathrm{~nm}$.

Die Auswertung aller vorliegenden Daten führte zu der folgenden, möglichen Struktur:<smiles>COc1ccc2c(c1)C(=O)C(CNC(C)=O)=N2</smiles>

Abb. 69: Nummerierungssystem von Produkt 4 
Formalname: $\quad N$-(5-Methoxy-3-oxo-3H-indol-2-ylmethyl)-acetamid

$\begin{array}{ll}\text { Aussehen: } & \text { gelblich } \\ \text { Fluoreszenz: } & \mathrm{Ex}=343, \mathrm{Em}=389 \mathrm{~nm} \\ \text { Rf-Wert } & 0,59 \text { in Ethylacetat / MetOH (9.5 : 0.5) } \\ \text { Masse } & 232 \\ \text { Molekülformel: } & \mathrm{C}_{12} \mathrm{H}_{12} \mathrm{~N}_{2} \mathrm{O}_{3}\end{array}$

EI-MS: m/z (\%) 232.1 (67), 190.1 (100), 175.0 (62), 161.0 (6), 118.0 (5), 77.0 (2), 63.0 (9), 43.0 (27)

CI-MS: m/z (\%) $233.1[\mathrm{M}+\mathrm{H}]^{+}(100), 250.2\left[\mathrm{M}+\mathrm{NH}_{4}\right]^{+}(10), 465.3\left[2 \mathrm{M}+\mathrm{NH}_{4}\right]^{+}(14)$.

${ }^{1}$ H-NMR (CD 3 OD, 600 MHz): $\delta=7.15$ (dd, 1 H, 8.7 u. 2.6 Hz, 4-H), 6.96 (d, 1-H, $2.7 \mathrm{~Hz}$, 3-H), 6.85 (d, 1-H, 8.8 Hz, 3-H), 4.15 (brs, 1-H, NH), 3.70 (s, 3 H, OCH $\mathrm{OCH}_{3} 1.92$ (s, 3-H, $\mathrm{CH}_{3}$ ). 


\section{$\underline{\text { 3.4.4 Produkt } 5}$}

Wie schon unter Punkt 3.4.3 erwähnt wurden zwei Produkte isoliert, wobei Produkt 5 den geringsten Mengenanteil hatte.

Dementsprechend konnten lediglich Massenspektren aufgenommen werden, da die Nachweisbarkeitsgrenze für die NMR-Spektren unterschritten war.

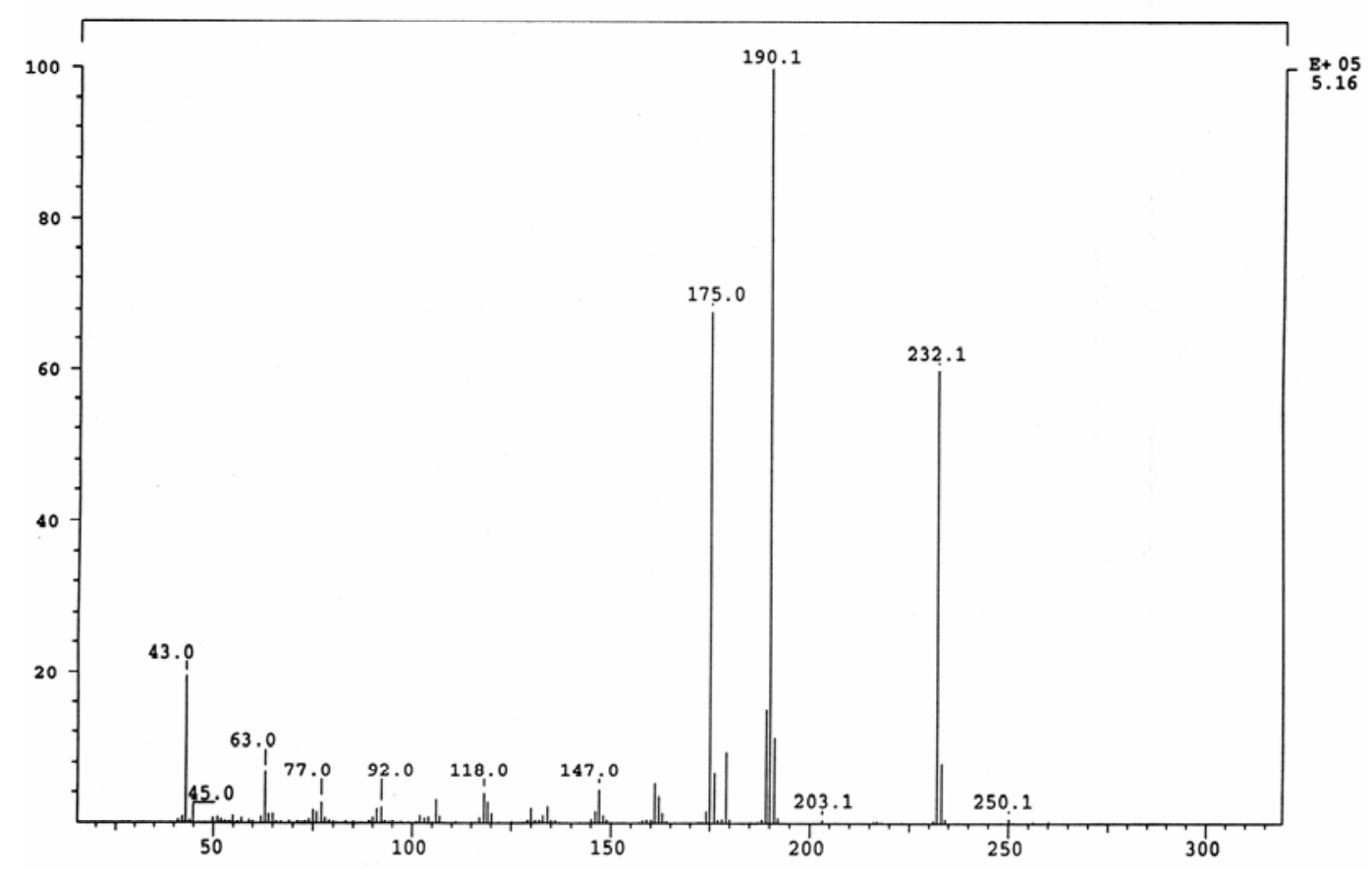

Abb. 70: Massenspektrum (EI-MS, 70 eV) von Produkt 5

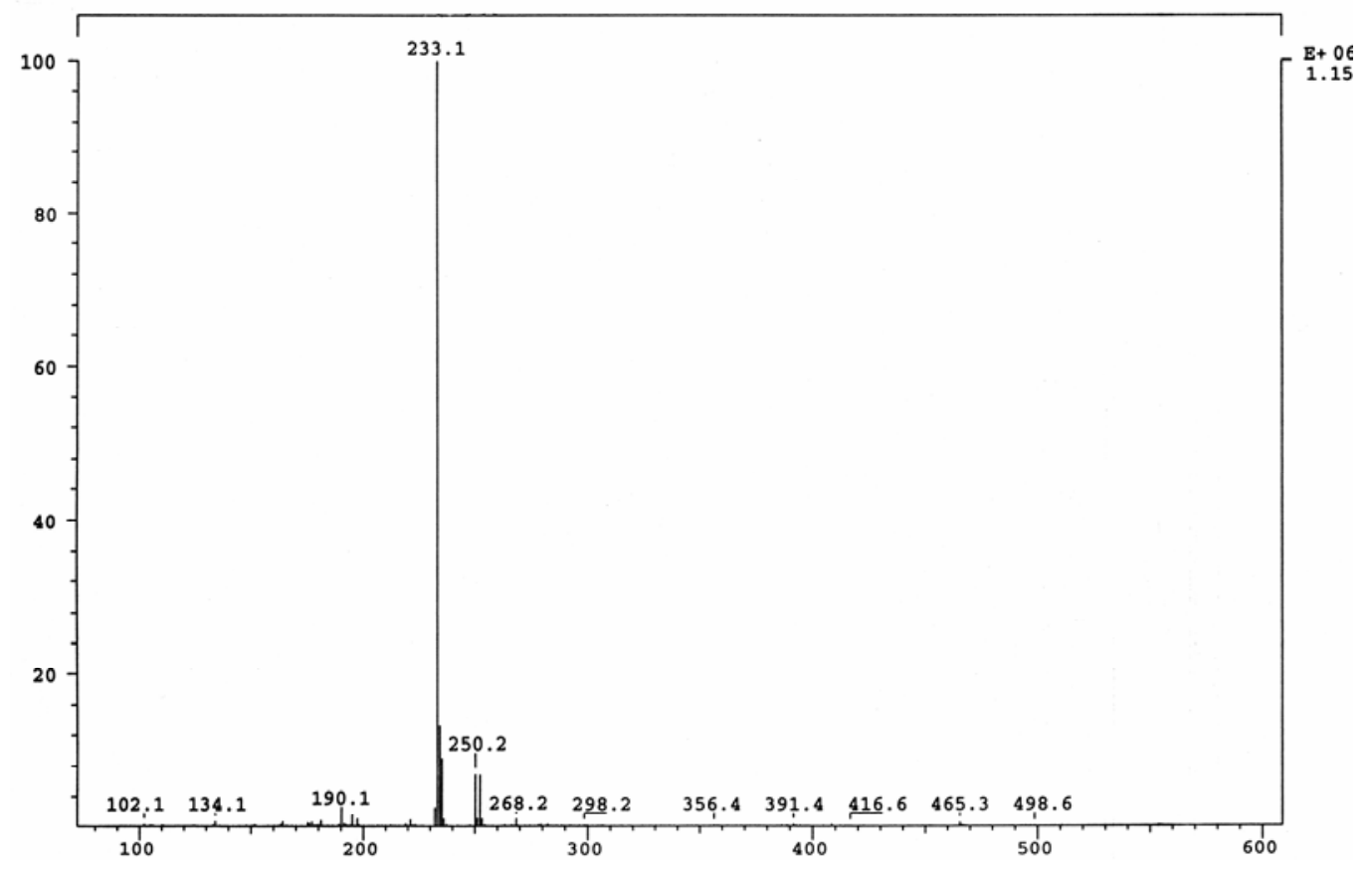

Abb. 71: Massenspektrum (CI-MS, 70 eV) von Produkt 5 (H+) 


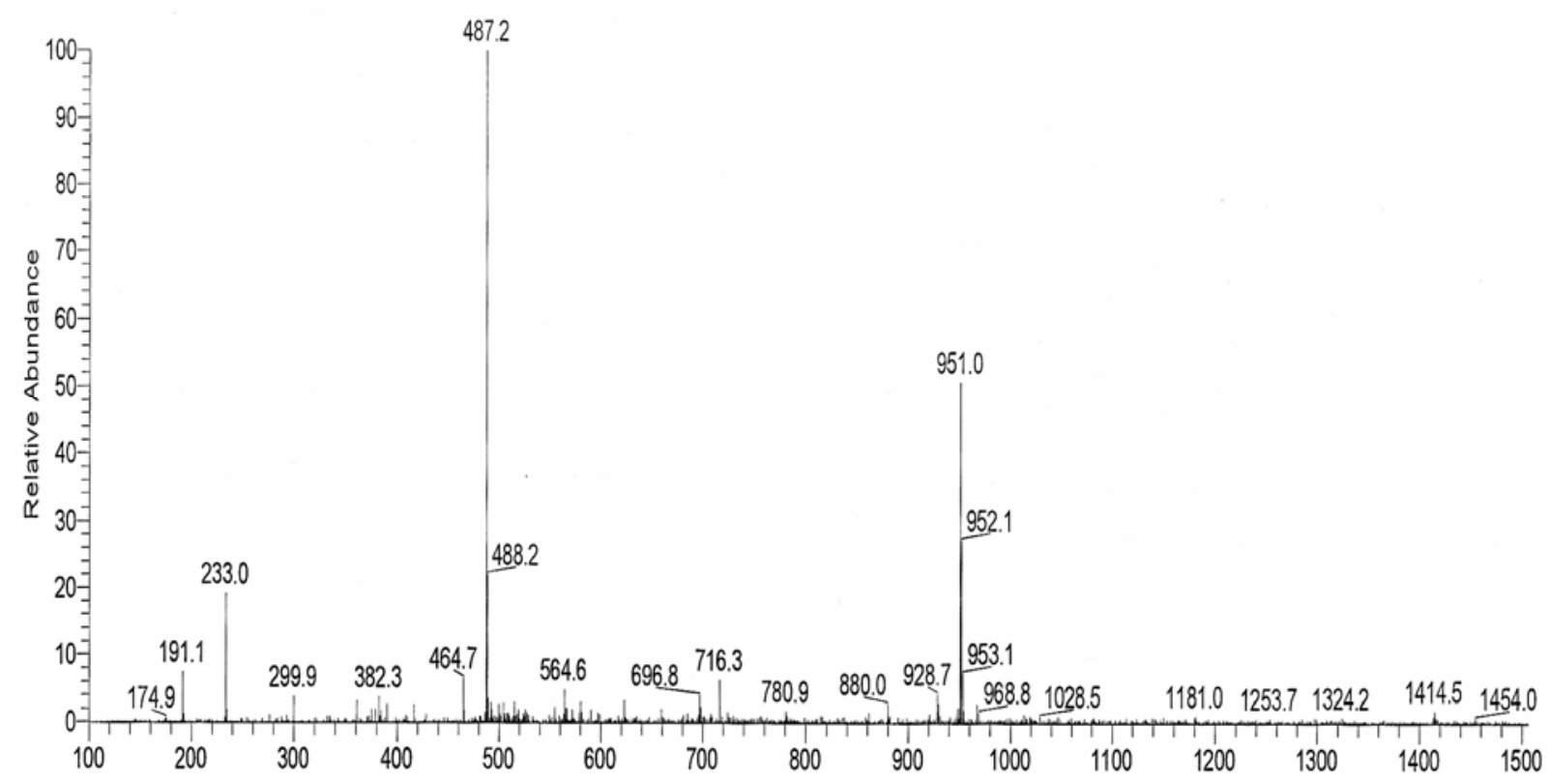

Abb. 72: Massenspektrum (ESI-HRMS, 70 eV) von Produkt 5 (H+)

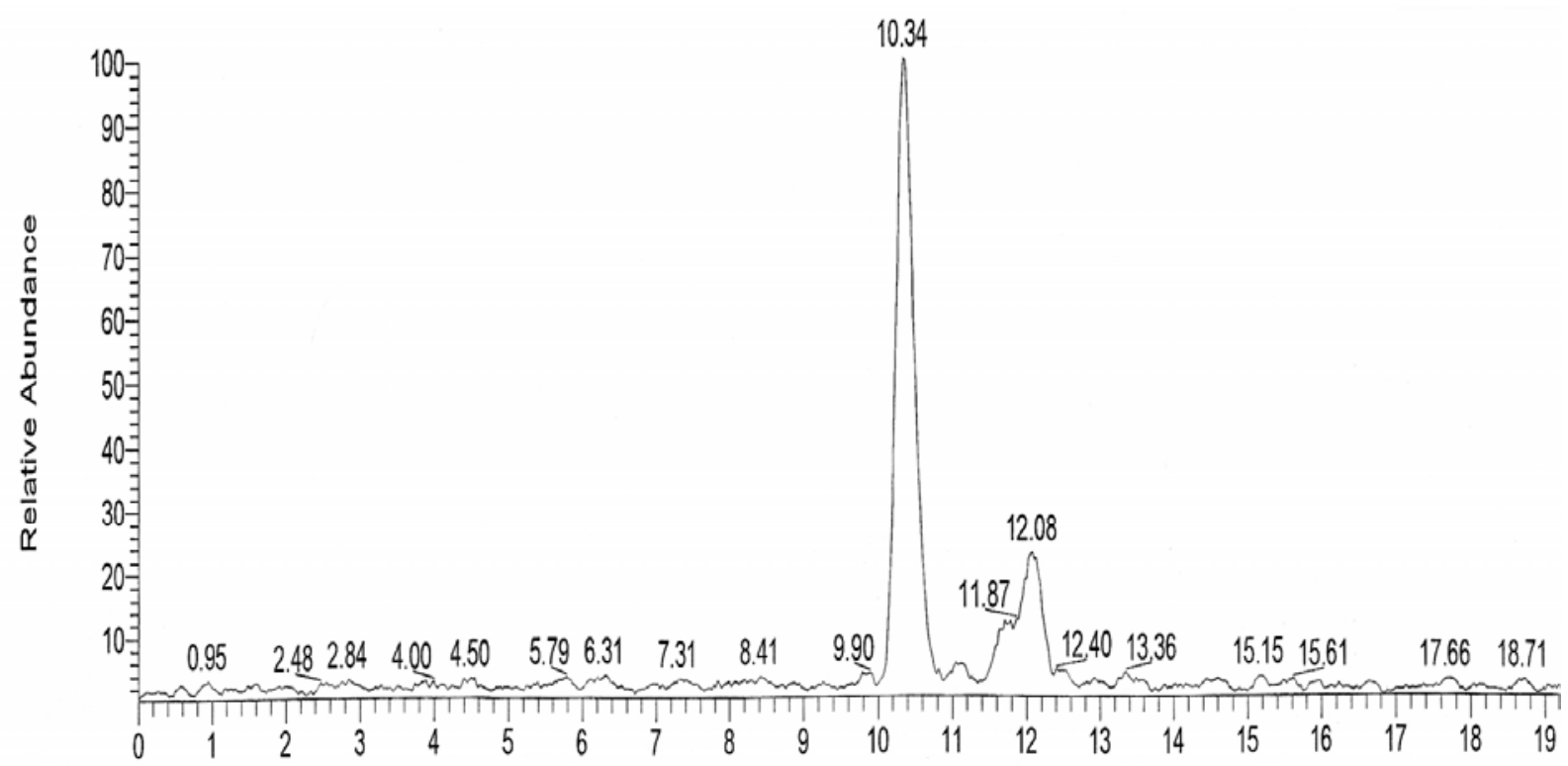

Abb. 73: HPLC-MS ( $\mathrm{MeOH} / \mathrm{H}_{2} \mathrm{O}, 10-100 \% \mathrm{MeOH}, 0-20$ min) von Produkt 5 


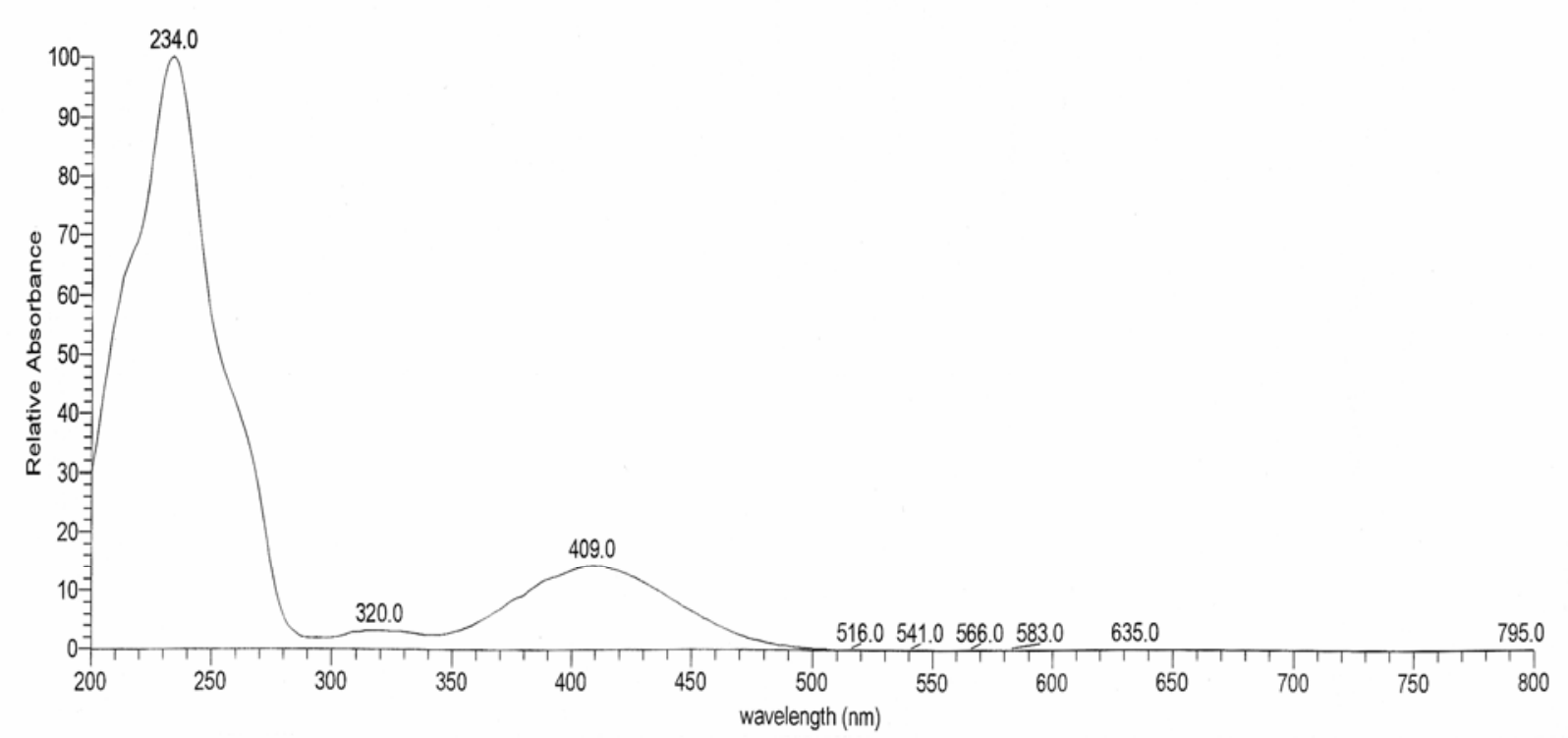

Abb. 74: UV-Spektrum von Produkt 5

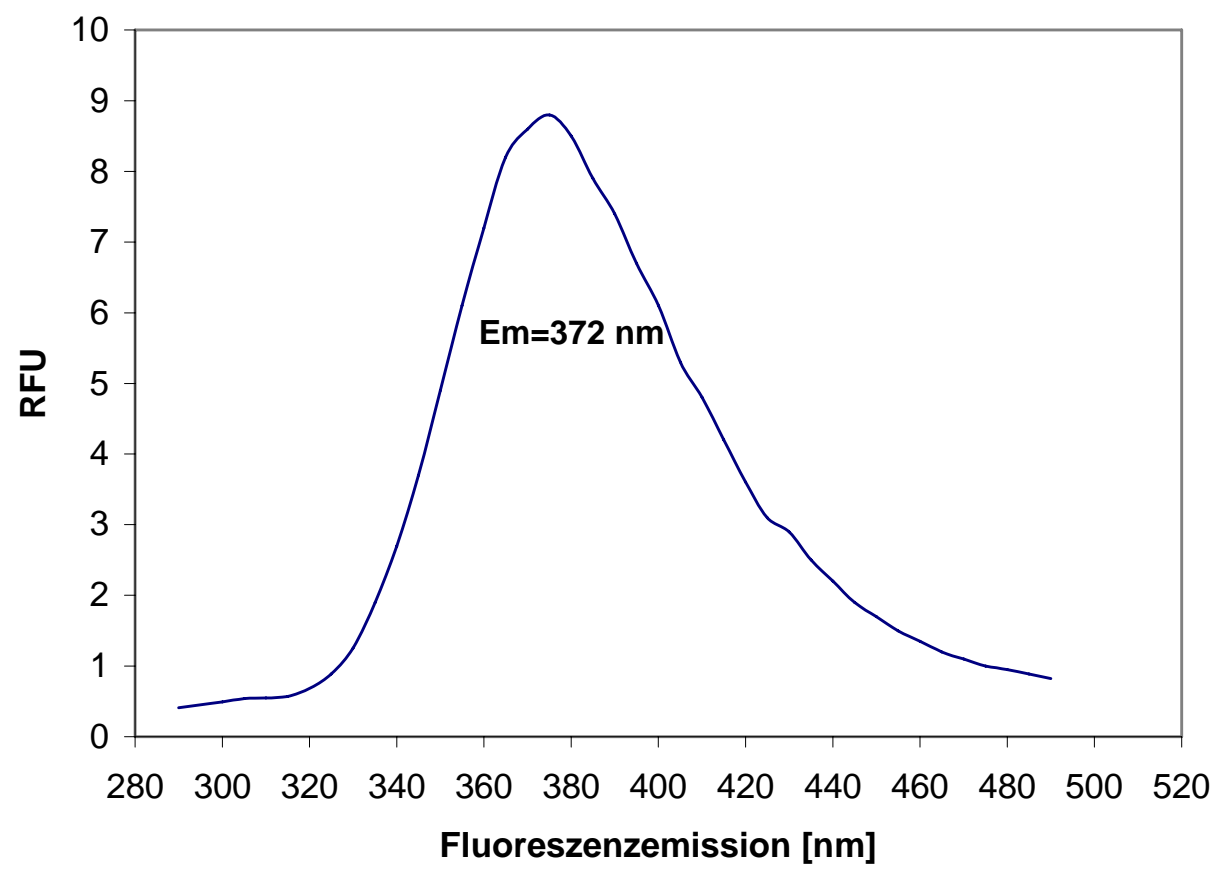

\section{Abb. 75: Emissionsspektrum von Produkt 5}

Da sich das Excitationsmaximum unterhalb von $287 \mathrm{~nm}$ befand, konnte ein Excitationsspektrum nicht aufgenommen werden. 
Die vermutliche chemische Struktur beruht auf dem Vergleich der Massenspektren mit denen von Produkt 4 und Produkt 2. In Analogie zu Produkt 2 ist zu vermuten, dass auch von Produkt 5 E,Z-Isomere vorkommen.<smiles>COc1ccc2c(c1)C(=O)/C(=C/NC(C)=O)N2</smiles>

Produkt 5a<smiles>COc1ccc2c(c1)C(=O)/C(=C\NC(C)=O)N2</smiles>

Produkt 5b

Abb. 76: Nummerierungssystem der E,Z-Isomere von Produkt 5

Formalname: $\quad N$-(5-Methoxy-3-oxo-1,3-dihydro-indol-2-ylidenemethyl)-acetamid

$\begin{array}{ll}\text { Aussehen: } & \text { rötlich } \\ \text { UV: } & \lambda \max 234,320 \text { und } 409 \mathrm{~nm} \\ \text { Fluoreszenz: } & \mathrm{Ex}=--, \mathrm{Em}=372 \mathrm{~nm} \\ \text { Rf-Wert } & 0,59 \text { in Ethylacetat / MetOH (9.5 : 0.5) } \\ \text { Masse } & 232 \\ \text { Molekülformel: } & \mathrm{C}_{12} \mathrm{H}_{12} \mathrm{~N}_{2} \mathrm{O}_{3}\end{array}$

EI-MS: m/z (\%) 232.1 (60), 190.1 (100), 175.0 (67), 147.0 (4), 118.0 (4), 92.0 (3), 77.0 (3), $63.0(6), 43.0(19)$

CI-MS: m/z (\%) $233.1[\mathrm{M}+\mathrm{H}]^{+}(100), 250.2\left[\mathrm{M}+\mathrm{NH}_{4}\right]^{+}(6), 465.3\left[2 \mathrm{M}+\mathrm{NH}_{4}\right]^{+}(1)$. 


\section{Diskussion}

Die sich bereits zuvor andeutenden Unterschiede in der Oxidationschemie zwischen Melatonin und anderen strukturverwandten Indolaminen (Poeggeler et al., 2002) verlangt nach einer weiterführenden Analyse. Ein entscheidender Fortschritt in der hier vorgelegten Untersuchung besteht darin, dass sich diese Verschiedenheiten nicht allein als solche der Reaktivität erwiesen haben, sondern in z. T. überraschendem Umfang als Abweichungen im Produktspektrum, was naturgemäß auf strukturspezifische Reaktionsmöglichkeiten zurückgeführt werden muss, die beim Melatonin offenbar viel stärker eingeschränkt sind als bei anderen Indolaminen und bei denen kleine Änderungen der Substituenten erhebliche Konsequenzen nach sich ziehen.

Solche Unterschiede in den Reaktionsmöglichkeiten dürften unter dem Aspekt pharmakologischer Anwendung von entscheidender Bedeutung sein, was im guten Einklang mit der ausgezeichneten Verträglichkeit von Melatonin selbst in hohen Dosen steht (Weishaupt et al., 2006). Da Melatonin in erster Linie - teils über die Zwischenstufe c3OHM, teils direkt - zum AFMK verstoffwechselt wird (Hardeland et al., 2004), entsteht hier erneut eine protektive Substanz (Tan et al., 2001, Tan 2002). Konsequenterweise stellt sich hier sodann auch die Frage, in welcher Weise AFMK weiter zu reagieren im Stande ist.

Im Einzelnen zeigte sich, dass - im Gegensatz zur präferierten Pyrrolringspaltung beim Melatonin in verschiedenen Oxidationssystemen - die anderen Indolamine nicht nur zu weiteren Produkten führen, sondern dass dies in hohem Maße auch vom verwendeten Oxidans abhing.

Sinnvollerweise sind die Vergleiche auf das Vorhandensein der relevanten Substituenten zu beziehen, d.h. Hydroxy- oder Methoxygruppen an C-5 bzw. C-6 und N-Acetylreste.

Dabei zeigte sich schon, dass das Fehlen der Acetylgruppe im 5-Methoxytryptamin (5-MT) erhebliche Auswirkungen besaß. Bei 5-MT entstanden im Protoporphyrin-IX-katalysierten System wesentlich mehr Produkte (Abb. 8) als bei Melatonin (Abb. 5). Des weiteren ergab sich in diesem System, dass 5-MT (Abb. 9) einen höheren Umsatz hatte als Melatonin (Abb. 7). 
Im Gegensatz dazu zeigen die beiden Indolamine Serotonin und $N$-Acetylserotonin, dass die $N$-Acetylierung der Seitenkette im Protoporphyrin-IX-System zu einer Beschleunigung des oxidativen Umsatzes führte (Abb. 18, 15). Diese Beobachtungen konnten ebenso im Häminkatalysierten $\mathrm{H}_{2} \mathrm{O}_{2}$-System gemacht werden (Poeggeler et al., 2002). Diese unterschiedlichen Reaktionen der Indolamine in den einzelnen Oxidationssystem lassen erkennen, dass nicht alle Aspekte des einen auf das andere System übertragen werden können.

Die einzelnen Substituenten führen zu unterschiedlichen Konsequenzen.

Das Ersetzen der 5-Methoxy-Gruppe im Melatonin durch eine 5-Hydroxy-Gruppe zum $N$-Acetylserotonin bewirkte, dass im Chromatogramm (Abb. 13) 3 weitere, möglicherweise kynurische Produkte auftraten. Im Vergleich erschienen im Chromatogramm der 5-MTOxidation (Abb. 8) mehr als doppelt so viele Banden.

Der Umsatz von NAS im Protoporphyrin-IX-System (Abb. 15) ist ebenso wie bei 5-MT (Abb. 9) deutlich erhöht. Nahezu 3/4 des eingesetzten NAS werden hierbei oxidiert. Dies spiegelt sich ebenso in der Umsatzanalyse im ABTS ${ }^{+}{ }^{-}$Reduktionssystem (Abb. 37) wider.

Dagegen scheint der Einfluss der 5-Hydroxylierung am Benzolring bei den einzelnen Indolaminen unterschiedliche Effekte zu haben. So der Umsatz von NAS schneller als der von NAT $(15,12)$, während Serotonin im geringeren Umfang oxidiert wird als Tryptamin (Abb. $18,20)$.

Dass dies zum Teil Reaktionssystem-bedingte Unterschiede sind, lässt sich leicht durch den Vergleich des Protoporphyrin-IX-Systems mit dem ABTS $^{\bullet+}$-Reduktionssystems erkennen. Wie schon zuvor erwähnt, zeigt Serotonin im Gegensatz zu Tryptamin eine deutliche Verminderung des oxidativen Umsatzes. Dies spiegelt sich aber im ABTS $^{\circ}$-Assay nicht unbedingt wieder. Bei dem Vergleich der Stöchiometrie der beiden Indolamine zeigt Serotonin einen höheren Wert als Tryptamin $(34,32)$, ebenso wie bei der konzentrations- und zeitabhängigen $\mathrm{ABTS}^{{ }^{+}}{ }^{+}$-Reduktionsanalyse $(33,31)$.

Die Vergleiche zwischen den Indolaminen müssen auf dem Hintergrund der in den Reaktionssystemen gebildeten Indolylradikalen gesehen werden wie auch der Interaktion zwischen einem $N$-Acetylrest und dem Pyrrolring, letzteres - nicht von vornherein vorhersehbar - wird bereits durch die Bildung von c3OHM demonstriert. Hinsichtlich der 
Indolylradikale ist festzuhalten, dass am Benzolkern unsubstituierte Indolamine notwendigerweise leicht C-zentrierte Radikale bilden, am Benzol hydroxylierte sowohl O- als auch C-zentrierte Radikal ergeben, während sie bei den methylierten Analogen nicht möglich bzw. nicht begünstigt ist, so dass sie präferenziell zu $N$-zentrierten Radikalen (Hardeland et al., 1993) führen. O-zentrierte Radikale tendieren zur Propagation radikalischer Reaktionsketten (Hardeland, 2005), was für Serotonin als prooxidatives Verhalten offenkundig wird (Tan et al., 1993). C-zentrierte Radikale begünstigen Dimerisierungen, was ebenfalls für Serotonin und $N$-Acetylserotonin gezeigt wurde (Huether et al., 1997; Behrends et al., 2004; Behrends \& Hardeland unpubl. Daten).

\section{$\underline{\text { 6-Methoxymelatonin }}$}

Eine Sonderstellung im Vergleich der Indolamin-Oxidation nimmt 6-Methoxymelatonin ein. Diese kommerziell nicht erhältliche Substanz wurde entwickelt, um einen melatoninergen Agonisten (Dubocovich et al., 1997) zu erhalten, der jedoch aufgrund seines zusätzlichen Methoxy-Substituenten am C6-Atom nicht mehr dem hepatischen Abbau durch $\mathrm{P}_{450}$-Monoxygenase unterliegt und daher eine erheblich längere Halbwertszeit als Melatonin besitzen muss. Dies würde zugleich bedeuten, dass 6-Methoxymelatonin ebenfalls erheblich länger als Radikalfänger zur Verfügung stünde, wenn es pharmakologisch gegeben würde.

Dennoch erscheint der Einsatz von 6-Methoxymelatonin als Antioxidans fragwürdig. In einer diesbezüglichen Untersuchung zeigte sich, dass 6-Methoxymelatonin in geringeren Konzentrationen weniger, in höheren Konzentrationen eine größere Wirksamkeit besaß als Melatonin (López-Burillo et al., 2003).

Dieser Vorbehalt ergibt sich aus den hier gezeigten Unterschieden in der Oxidationschemie zwischen 6-Methoxymelatonin und Melatonin. Wie zu erwarten, wird 6-Methoxymelatonin aufgrund der zweiten reaktivitätssteigernden Methoxygruppe schneller umgesetzt (Abb. 21). Diese Reaktionssteigerung führt jedoch dazu, dass 6-Methoxymelatonin bereits durch Hydroxylradikale-unabhängige Reaktionen größtenteils umgesetzt wird, wie das Häminkatalysierte Oxidationssytem in Gegenwart von DMSO erweist (Abb. 25). Dieses Ergebnis könnte die geringer Effizienz von 6-Methoxymelatonin in niedriger Dosis (López.Burillo et al., 2003) erklären. 
Wiewohl aus 6-Methoxymelatonin ein auffälliges, offenbar kynurisches Produkt entsteht, welches nach Analogieschluss $N^{1}$-Acetyl- $N^{2}$-formyl-5,6-dimethoxykynuramin (AFDK) sein müsste, sind dennoch Unterschiede in der Produktbildung im Vergleich zu Melatonin vorhanden, was sich in zusätzlichen Produktbanden äußert (Abb. 23, Tab. 8).

Bevor 6-Methoxymelatonin überhaupt eingesetzt werden könnte, müssten die Eigenschaften dieser zusätzlichen Produkte geklärt werden.

Vergleiche mit anderen 6-substituierten Melatonin-Derivaten sind bislang - abgesehen von Rezeptoraffinitätsstudien (Dubocovich et al., 1997) - nur anhand von Chemilumineszenzdaten möglich (Poeggeler et al., 2002).

6-Hydroxymelatonin wurde im Hämin-katalysierten System wesentlich langsamer umgesetzt als Melatonin und wie hier belegt, 6-Methoxymelatonin. 6-Chloromelatonin hingegen zeigt ähnliche Charakteristika wie Melatonin, sowohl hinsichtlich der Kinetik als auch der Photonenausbeute. Die wesentlich geringeren Lichtemissionsraten, die mit 6-Methoxymelatonin gemessen werden (Abb. 21, 22), lassen sich eher nicht auf nichtlumineszente Konkurrenzreaktionen zurückführen, da der kynurische Produktanteil erheblich ist. Der Unterschied müsste also vor allem in einer verminderten Stärke des chemilumineszenten Emitters zu suchen sein. Derartige Phänomene sind nicht von vornherein durch die Elektronenverteilung im Edukt zu erklären, sondern resultieren weitgehend aus der Stabilität des aktiven Carbonyls im lumineszenten Intermediats. Eine etwas längere Lebensdauer bewirkt Energiedissipation als Wärme und folglich geringere Lumineszenz, was beim Vergleich von Indolen oft festzustellen ist (Hardeland, pers. Mitt.).

\section{Produkte von AFMK}

Eine der wichtigsten Erkenntnisse dieser Arbeit war, dass die Erweiterung der Radikalfängerkaskade von Melatonin größer ist, als aufgrund vorangegangener Untersuchungen (Tan et al., 2001) vorhergesagt werden konnte.

Das hierbei verwendete ABTS-Kationradikal-System erwies sich als besonders nützlich für die Identifizierung sequentieller Schritte bei der Produktbildung aus Melatonin (Tan et al., 2003).

Eine Auswertung der Stöchiometrie ist aber nur dann sinnvoll, wenn das ABTS $^{{ }^{+}}$während eines Experiments nicht vollständig verbraucht wird, sondern vielmehr im Überschuss vorliegt. 
Die Ergebnisse dieser Arbeit haben gezeigt, dass bei einem Verhältnis von 1:40 etwa 10 Radikale reduziert werden. Dieses Resultat deutet auf eine viel höher antioxidative Wirksamkeit von Melatonin hin als zuvor erwartet.

Es zeigte sich, dass bereits in den ersten 15 min circa 7 ABTS-Radikale reduziert wurden, bei einer Konzentration von $1 \mu \mathrm{M}$ Melatonin und $40 \mu \mathrm{M} \mathrm{ABTS}^{\bullet^{+}}$(Abb. 27, 28).

Diese Tatsache ist unter physiologischen Bedingungen betrachtet kein schlechter Wert, da AFMK relativ leicht mit ${ }^{\circ} \mathrm{OH}$ reagiert (Burkhardt et al., 2001, Tan et al., 2001) und auch bereitwillig in Reaktionssystemen erzeugte Radikale eliminiert.

Bei der Produktanalyse konnte gezeigt werden, dass es sich bei den Oxidationsprodukten um eine völlig neue, noch nicht zuvor beschriebene Gruppe von Substanzen handelte. Anmerkend sollte hier erwähnt werden, dass der AFMK Metabolit AMK nicht innerhalb der Nachweisgrenze zu detektieren war.

Dies ist nicht darauf zurückzuführen, dass eventuell entstehendes AMK schneller oxidiert wird als aus AFMK nachgebildet. Vielmehr wurden auch keine aus AMK im ABTS ${ }^{+}$-System gebildeten Oxidationsprodukte (Than et al., 2006) gefunden.

Die entstandenen Produkte können als Ergebnis einer Art von Rezyklisierung des AFMK angesehen werden, wobei es zur Bildung von 3-Indolinonen kommt. Die Besonderheit hierbei ist, dass die für Kynuramine bzw. Kynurenine typische Oxo-Gruppe erhalten bleibt, jedoch die Seitenkette am C2-Atom des neu gebildeten heteroaromatischen Ringes erscheint und um ein C-Atom verkürzt ist, da sich die $N$-Formyl-Gruppe von AFMK nicht an der Rezyklisierung beteiligt.

Diese C2-substituierten 3-Indolinone sollten nicht mit C3-substituierten 2-Indolinonen verwechselt werden, welche in anderen Reaktionssystemen beim Fangen von $2{ }^{\circ} \mathrm{OH}$ durch Melatonin entstehen, z.B. durch Ketonbildung aus 2-Hydroxymelatonin (Hardeland, 2005; Agozzino et al., 2003).

Unter welchen Voraussetzungen sich diese neue Substanzen auch unter physiologischen Bedingungen bilden, muss noch untersucht werden. Des weiteren müssen homologe Reaktionen von AFMK mit anderen elektronenabstrahierenden freien Radikalen analysiert werden.

Diese neu entdeckten C2-substituierten 3-Indolinone können auch in einem anderen Zusammenhang von Bedeutung sein, da ähnliche Substanzen, die ebenfalls eine aliphatische Seitenkette am C2-Atom tragen, speziell für die Suche nach Melatonin-Rezeptor-Liganden 
und Melatonin-verwandten Antioxidantien synthetisiert worden sind (Spadoni et al. 1998, 2001, 2006; Mor et al., 2004). Der große Unterschied zu jenen ist das Vorhandensein einer Carbonyl-Gruppe am C3-Atom und die Verkürzung der aliphatischen Seitenkette um ein C-Atom.

Hieraus ergibt sich die interessante Frage, ob die neu entdeckten Substanzen 2a/b und 3 aus AFMK ebenfalls Eigenschaften von Rezeptor-Liganden aufweisen.

Die beiden Substanzen 2a und 2b stellen E,Z-Isomere dar, welche zur selben Zeit während der Isolierung vorhanden waren. Das E-Isomer (2b), das durch die Wasserstoffbückenbindung zwischen dem Acetamido-Stickstoff und dem Sauerstoff an C3 stabilisiert wird, stellt vermutlich die bevorzugte Struktur dar und ist daher auch offensichtlich in höheren Mengen als $2 \mathrm{a}$ vorhanden. Die Reihenfolge bei der Bildung der ungesättigten Produkte $2 \mathrm{a} / \mathrm{b}$ und der gesättigten C2-hydroxylierten Substanz 3 ist hierbei klar vorgegeben. Im ABTS ${ }^{\bullet^{+}-}$ Reaktionssystem ergab sich das Produkt 3 aus dem Racemat der Produkte 2a/b, jedoch nicht im umgekehrten Fall. Des weiteren führten Versuche, das Wasser mit konz. $\mathrm{H}_{2} \mathrm{SO}_{4}$ aus dem Produkt 3 zu entfernen, nicht zu den Produkten 2a/b, sondern zu ganz anderen Substanzen.

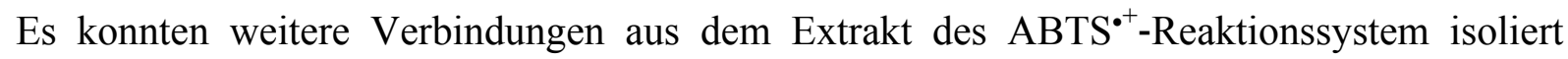
werden, welche nach Ergebnissen aus der Massenspektrometrie deformyliert sein müssten. Ob sich diese farbigen, fluoreszierenden Produkte aus der Deformylierung der Produkte $2 \mathrm{a} / \mathrm{b}$ oder auf anderem Wege z. B. aus Produkt 3 ergeben, ist zur Zeit noch unklar. Die wahrscheinlichen Strukturen $(4,5 \mathrm{a} / \mathrm{b})$ sind in Abb. 69, 76 wiedergegeben, wobei $5 \mathrm{a} / \mathrm{b}$ ebenfalls E,Z-Isomere darstellen.

Mit Blick auf die Radikalfängerkaskade sollte die Bildung von 3-Indolinon 2a/b durch die Rezyklizierung eines Intermediats von AFMK schon erheblich zur Fangkapazität beitragen.

Bei der Oxidation von Melatonin zu AFMK können vier Radikale im ABTS ${ }^{+}$-System gefangen werden (Tan et al. 2003). Bei der Reaktion von AFMK zu 2a/b werden weitere 4 Elektronen verbraucht, wobei der genaue Mechanismus noch nicht genau geklärt ist. Diese Bilanz von 8, welche pro Melatonin-Molekül gefangen werden, erklärt noch nicht die gemessenen $10 \mathrm{ABTS}^{{ }^{+}}$(Abb. 27, 28). Daher bleibt die Frage, welche zusätzlichen Schritte zur Reduktion der verbleibenden Radikale beitragen. Die Umwandlung von $2 \mathrm{a} / \mathrm{b}$ zum Indolinon 3 kann möglicherweise als Wasseraufnahme verstanden werden und würde daher nicht in der Bilanz erscheinen.

Jedoch erscheint die Anwesenheit einer höheren Menge von Indolinon 3 und der offenbaren 
Abwesenheit von $2 \mathrm{a} / \mathrm{b}$ in einem $\mathrm{H}_{2} \mathrm{O}_{2}$-haltigen Reaktionssystem (Rosen et al., 2006), bei vorsichtiger Betrachtung, auf die Beteiligung einer Redox-Reaktion hinzuweisen.

Substanz 3 zeichnete sich durch eine relativ geringe Reaktivität im ABTS-RückreduktionsAssay aus. Diese Substanz blieb über längere Zeit im Reaktionssystem stabil und repräsentierte das Hauptprodukt aus dem Umsatz von AFMK. Die Reaktionskaskade kann daher unter Entstehung von Substanz 3 in dem ABTS $^{{ }^{+}}$-System terminiert werden. Unter dieser Annahme müssten die zwei noch nicht erklärten zusätzlich gefangenen Radikale auf eine Nebenreaktion zurückgeführt werden.

Dieses Problem kann zum jetzigen Zeitpunkt nicht leicht erklärt werden, insbesondere weil die Substanzen 2a/b relativ instabil sind. Dies gilt in noch höheren Maße für die Substanzen 4 und $5 \mathrm{a} / \mathrm{b}$, die nur in sehr geringen Mengen isoliert werden konnten. Ferner ist davon auszugehen, daß die Stereoisomere ineinander überführt werden können.

Da eine NH-Gruppe im Heterozyklus der Verbindung 5a/b vorhanden ist, ist in Analogie zu den reaktiven Indolaminen mit einer Methoxy-Gruppe an C5 eine Radikalentgiftung durch Elektronendonatoren aus dem einsamen Elektronenpaar denkbar.

Diese Befunde einer Termination von Radikalreaktionen in einer antioxidativen Kaskade im $\mathrm{ABTS}^{{ }^{+}}{ }^{-}$-System lässt sich nicht ohne weiteres auf die komplexe Situation in vivo übertragen. Substanz 3 reagiert mit dem Hydroxyl-Radikal im ABTS ${ }^{\bullet+}$-Kompetitions-Assay (Rosen et al., 2006). Daher könnte die antioxidative Kaskade in vivo sogar noch unter Oxidation des Indolinons 3 fortgeführt werden.

Die hier vorgelegten Befunde zur Oxidation von Melatonin und den aus ihm durch Radikalreaktionen hervorgehenden Produkten unterstreichen die Besonderheit des Melatonins im Vergleich $\mathrm{zu}$ anderen Indolen, was im Zusammenhang mit seiner therapeutischen Anwendung von Interesse ist. 


\section{Zusammenfassung}

Eine Vergleichsanalyse strukturverwandter Indolamine von Melatonin zeigte, dass bereits kleine Änderungen der Substituenten erhebliche Konsequenzen in der Oxidationschemie nach sich ziehen.

Analysiert wurden hierbei die Substitutionen von Hydroxy- oder Methoxygruppen an C-5 bzw. C-6 und einem aliphatischen $N$-Acetylrest. Im Einzelnen wurden Tryptamin, 5-Methoxytryptamin, $N$-Acetyltryptamin, Serotonin, $N$-Acetylserotonin, Melatonin und 6-Methoxymelatonin in folgenden Oxidationssystemen verglichen: Photooxidation mit Protoporphyrin-IX, Hämin-katalysiertes $\mathrm{H}_{2} \mathrm{O}_{2}$-System und Elektronendonation an ABTSKationradikalen $\left(\mathrm{ABTS}^{{ }^{+}}\right)$.

5-Methoxytryptamin und $N$-Acetylserotonin zeigten einen beschleunigten Umsatz im Protoporphyrin-IX-System. Im ABTS ${ }^{+}{ }^{-}$-System hingegen ist 5-Methoxytryptamin langsamer als Melatonin. Im Vergleich zu $N$-Acetyltryptamin wurde $N$-Acetylserotonin schneller oxidiert. Die Reaktion von Serotonin verlief langsamer als die von Tryptamin. 5-Methoxytryptamin und Tryptamin ergaben eine erheblich größere Zahl an Oxidationsprodukten als Melatonin.

Der Sonderfall des nicht natürlichen 6-Methoxymelatonin zeigte, dass die Einführung der zusätzlichen Methoxygruppe an C6 zu einer Reaktivitätssteigerung und damit schnellerem Umsatz führte, dies bereits in Hydroxylradikal-unabhängigen Reaktionen. Im Produktspektrum konnten zusätzliche Substanzen detektiert werden, deren nähere Untersuchung noch aussteht. Diese Ergebnisse einer abweichenden Oxidationschemie lassen einen therapeutischen Einsatz von 6-Methoxymelatonin, zum jetzigen Zeitpunkt, ohne Kenntnis der zusätzlichen Produkte, noch nicht angeraten erscheinen.

Das wichtigste Resultat der hier vorgenommen Produktanalyse führt zu einer Erweiterung der Radikalfängerkaskade von Melatonin.

Zuvor war angenommen worden, dass diese mit der Bildung von $N^{1}$-Acetyl- $N^{2}$-formyl-5methoxykynuramin (AFMK) endet. Es wird nunmehr gezeigt, dass AFMK durch radikalische Mechanismen zu Substanzen umgesetzt wird, die einer bislang unbekannten Klasse von Melatonin-Metaboliten angehören. Sie entstehen ebenfalls beim Umsatz von Melatonin als Ausgangsprodukt. Hierbei handelt es sich um C2-substituierte 3-Indolinone, welche jede für sich eine chemisch neue Verbindung darstellt. 
Eine stöchiometrische Verbrauchsanalyse in $\mathrm{ABTS}^{\bullet+}$-Reduktionssystem zeigt, dass bis zu den neuen Produkten insgesamt 10 Radikale eliminiert werden. Zuvor war nur ein Verbrauch von 4 Radikalen bis zum AFMK bekannt gewesen.

Bei den in diesem Prozess neu entstanden Produkten handelt es sich um N-(1-Formyl-5methoxy-3-oxo-2,3-dihydro-1H-indol-2-ylidenmethyl)-acetamid [Produkt 2] und $\mathrm{N}$-(1Formyl-2-hydroxy-5-methoxy-3-oxo-2,3-dihydro-1H-indol-2-ylmethyl)-acetamid [Produkt 3]. Die neuen Substanzen können als ein Art von Rezyklisierungsprodukte des AFMK angesehen werden und bilden zum Teil E,Z-Isomere [Produkt 2a/b]. Bei zwei weiteren Verbindungen [Produkt 4 und Produkt 5] handelt es sich nach Daten der Massenspektroskopie offenbar um deformylierte Analoge. Im ABTS ${ }^{+}$-Reaktionssystem ergab sich das Produkt 3 aus dem Racemat der Produkte 2a/b, jedoch nicht umgekehrt.

Die Ergebnisse dieser Arbeit unterstreichen die außergewöhnlichen Redox-Eigenschaften von Melatonin einschließlich der aus ihm hervorgehenden Reaktionsprodukte. 


\section{Literatur}

Acuña-Castroviejo D., Coto-Montes A., Gaia Monti M., Ortiz G.G., Reiter R.J. (1997)

Melatonin is protective against MPTP-induces striatal and hippocampal lesions.

Life Sci. 60: L23-L29.

Agozzino P, Avellone G, Bongiorno D., Ceraulo L., Filizzola F., Natoli MC., Livrea M.A, Tesoriere L. (2003).

Melatonin: structural characterization of its non-enzymatic mono-oxygenate metabolite.

J. Pineal Res. 35:269-275.

Balzer I., Hardeland R. (1989)

Action of kynuramine in a dinoflagellate: stimulation of bioluminescence in Gonyaulax polyedra.

Comp. Biochem. Physiol. 94C: 129-132.

Behl C., Davis J.B., Lesley R., Schubert D. (1994)

Hydrogen peroxide mediates amyloid protein toxicity.

Cell 77: 817-927.

Behrends A., Hardeland R., Ness H., Grube S., Poeggeler B., Haldar C. (2004)

Photocatalytic actions of the pesticide metabolite 2-hydroxyquinoxaline: destruction of antioxidant vitamins and biogenic amines - implications of organic redox cycling.

Redox Rep. 9: 279-288.

Behrmann G. (1989)

Die Rolle des Metabolismus der aromatischen Aminosäure Tryptophan bei Gonyaulax polyedra.

Diplomarbeit, Göttingen.

Behrmann G., Uría H., Fuhrberg B., Poeggeler B., Hardeland R. (1995)

Melatonin terminiert photooxidative radikalische Reaktionsketten.

Verh. Dtsch. Zool. Ges. 88, Pt. 1: 94. 
Beyer H., Walter W. (1984)

Lehrbuch der Organischen Chemie. 20. Aufl.

Hirzel, Stuttgart.

\section{Bubenik G.A. (2001)}

Localization, physiological significance and possible clinical implications of gastrointestinal melatonin.

Biol. Signals Recept. 10: 350-366.

Bubenik G.A. (2002)

Gastrointestinal melatonin: localization, function, and clinical relevance.

Dig. Dis. Sci. 47: 2336-2348.

Bubenik G.A., Hacker R.R., Brown G.M., Bartos L. (1999)

Melatonin concentrations in the luminal fluid, mucosa, and muscularis of the bovine and porcine gastrointestinal tract.

J. Pineal Res. 26: 56-63.

Burkhardt S., Poeggeler B., Tan D.-X., Rosner C., Gruetzner T., Nitzki F., Schoenke M., Thuermann S., Reiter R.J., Hardeland R. (2001a)

Oxidation products formed from melatonin in various radical-generating systems.

In: Actions and redox properties of melatonin and other aromatic amino acid metabolites. (ed. by Hardeland R.), Cuvillier, Göttingen, pp. 9-22.

Burkhardt S., Reiter R.J., Tan D.-X., Hardeland, R., Cabrera J., Karbownik M. (2001b) DNA oxidatively damaged by chromium(III) and $\mathrm{H}_{2} \mathrm{O}_{2}$ is protected by the antioxidants melatonin, $N^{1}$-acetyl- $N^{2}$-formyl-5-methoxykynuramine, resveratrol and uric acid. Int. J. Biochem. Cell Biol. 33: 775-783.

\section{Cardinali D.P. (1981)}

Melatonin: A mammalian pineal hormone

Endocr. Rev. 2: 327-346 
Cryer B., Feldman M. (1998)

Cyclooxygenase-1 and cyclooxygenase-2 selectivity of widely used nonsteroidal antiinflammatory drugs.

Am. J. Med. 104: 413-421.

Dubocovich M.L., Masana M.I., Iacob S. and Sauri D.M. (1997)

Melatonin receptor antagonists that differentiate between the human Mella and Mellb recombinant subtypes are used to assess the pharmacological profile of the rabbit retina ML1 presynaptic heteroreceptor.

Naunyn Schmiedebergs Arch. Pharmacol. 355: 365-375

Ebadi M., Govitrapong P. (1986)

Neural pathways and neurotransmitters affecting melatonin

J. Neural. Transm. Suppl. 21: 125-155

Erel O. (2003)

A novel automated direct measurement method for total antioxidant capacity using a new generation, more stabile ABTS radical cation.

Clin. Biochem. 47: 277-285.

Erlich SS., Apuzzo ML. (1985)

The pineal gland: anatomy, physiology, and clinical significance.

J. Neurosurg. 63: 321-341

Esquifino A.I., Pandi-Perumal S.R., Cardinali D.P. (2004)

Circadian organization of the immune response: A role for melatonin.

Clin. App. Imunol. Rev. 4: 423.433.

Ferry G., Ubeaud C., Lambert P.H., Bertin S., Cogé F., Chromarat P., Delagrange R., Serkiz B., Bouchet J.P., Truscott T.J., Boutin J.A. (2005)

Molecular evidence that melatonin is enzymatically oxidized in a different manner than tryptophan. Investigation on both indoleamine-2,3-dioxygenase and myeloperoxidase.

Biochem. J. 388, Pt. 1: 205-215. 
Foote C.S. (1988)

Mechanistic characterization of photosensitized reactions.

In: Photosensitisation, molecular, cellular and medical aspects.

NATO ASI Ser., Vol. H 15 (ed. by Moreno G., Pottier R.H., Truscott T.G.), Springer, Berlin

- Heidelberg - New York - London - Paris - Tokyo, pp. 125-144.

Friebolin H. (1999)

Ein- und zweidimensionale NMR-Spektroskopie: eine Einführung. 3.Aufl.

Wiley-VCH, Weinheim.

Fuhrberg B., Hardeland R. (1994)

Oxidation of melatonin by free radicals: catalysis by hemin.

In: Cell biological problems in chronobiology.

(ed. by Hardeland R.), University of Göttingen, Göttingen, pp. 75-81.

Gawron E., Hardeland R. (1999)

Photocatalytic destruction of melatonin by chlorophyll.

In: Studies on antioxidants and their metabolites.

(ed. by Hardeland R.), Cuvillier, Göttingen, pp. 95-98.

Grace M.S. and Besharse J.C. (1993)

Solubilization and biochemical characterization of the melatonin deacetylase from Xenopus laevis retina.

J. Neurochem. 60: 990-999.

Guenther A.L., Schmidt S.I., Laatsch H., Fotso S., Ness H., Ressmeyer A.-R., Poeggeler B., Hardeland R. (2005)

Reactions of the melatonin metabolite AMK ( $\mathrm{N}^{1}$-acetyl-5-methoxykynuramine) with reactive nitrogen species: Formation of novel compounds, 3-acetamidomethyl-6-methoxycinnolinone and 3-nitro-AMK.

J. Pineal Res. 39: 251-260. 
Guerrero J.M., Reiter R.J. (2002)

Melatonin-immune system relationships.

Curr. Top. Med. Chem. 2: 167-179.

Hardeland R. (1996)

Photooxidation of melatonin: Catalysis by extracts from Pterygophora californica (Phaeophyceae).

In: Metabolism and cellular dynamics of indoles.

(ed. by Hardeland R.), University of Göttingen, Göttingen, pp. 149-152.

Hardeland R. (1997)

Melatonin: multiple functions in signalling and protection.

In: Skin cancer and UV radiation.

(ed. by Altmeyer P., Hoffmann K., Stücker M.), Springer, Berlin-Heidelberg, pp. 186-198.

Hardeland R. (1999)

Melatonin and 5-methoxytryptamine in non-metazoans.

Reprod. Nutr. Dev. 39: 399-408.

Hardeland R. (2005)

Antioxidative protection by melatonin. Multiplicity of mechanisms from radical detoxification to radical avoidance.

Endocrine 27: 119-130.

Hardeland R., Balzer I., Poeggeler B., Fuhrberg B., Uría H., Behrmann G., Wolf R., Meyer T.J., Reiter R.J. (1995)

On the primary functions of melatonin in evolution: Mediation of photoperiodic signals in a unicell, photooxidation and scavenging of free radicals.

J. Pineal Res. 18: 104-111.

Hardeland R., Coto-Montes A., Poeggeler B. (2003a)

Circadian rhythms, oxidative stress and antioxidative defense mechanisms.

Chronobiol. Int. 20: 921-962. 


\section{Hardeland R., Fuhrberg B. (1994)}

On the possible significance of melatonin oxidation in chronobiology.

In: Cell biological problems in chronobiology.

(ed. by Hardeland R.), University of Göttingen, Göttingen, pp. 100-103.

\section{Hardeland R., Fuhrberg B. (1996)}

Ubiquitous melatonin - presence and effects in unicells, plants and animals.

Trends Comp. Biochem. Physiol. 2: 25-45.

\section{Hardeland R., Fuhrberg B., Behrmann G., Balzer I. (1993a)}

Sleep-latency reducing pineal hormone melatonin as a scavenger of free radicals: hemin-catalysed formation of $\mathrm{N}^{1}$-acetyl- $\mathrm{N}^{2}$-formyl-5-methoxykynuramine.

Sleep Res. 22: 621.

\section{Hardeland R., Niebergall R., Schoenke M., Poeggeler B. (2001)}

Carbonate radicals as initiators of melatonin oxidation: Chemiluminescence and formation of oxidation products.

In: Action and redox properties of melatonin and other aromatic amino acid metabolites.

(ed. by Hardeland R.), Cuvillier, Göttingen, pp. 49-55.

\section{Hardeland R., Pandi-Perumal S.R. (2005)}

Melatonin, a potent agent in oxidative defense: Actions as a natural food constituent, gastrointestinal factor, drug and prodrug.

Nutr. Metab. (London), 2: article no. 22 [DOI: 10.1186/1743-7075-2-22]

\section{Hardeland R., Poeggeler B. (1995)}

Effect of oxidized glutathione on photooxidation of melatonin in a Gonyaulax extract/DMSO system.

In: Cellular rhythms and indoleamines.

(ed. by Hardeland R.), University of Göttingen, Göttingen, pp. 132-134. 


\section{Hardeland R., Poeggeler B. (2002)}

Chemistry and biology of melatonin oxidation.

In: Treatise on pineal gland and melatonin.

(ed. by Haldar C., Singaravel M., Maitra S.K.), Science Publishers (USA), Enfield, pp. 407422.

Hardeland R., Poeggeler B. (2003)

Non-vertebrate melatonin.

J. Pineal Res. 34: 233-241.

Hardeland R., Poeggeler B. (2005)

Melatonin: New aspects of its protective actions and novel metabolites.

In: Experimental endocrinology and reproductive biology.

(ed. by Haldar C., Singaravel M., Pandi-Perumal S.R., Cardinali D.P.), im Druck.

Hardeland R., Poeggeler B., Balzer I., Behrmann G. (1991)

Common basis of photoperiodism in phylogenetically distant organisms and its possible origins.

J. Interdiscipl. Cycle Res. 22: 122-123.

Hardeland R., Poeggeler B., Balzer I., Behrmann G. (1993b)

A hypothesis on the evolutionary origins of photoperiodism based on circadian rhythmicity of melatonin in phylogenetically distant organisms.

In: Chronobiology \& chronomedicine.

(ed. by Gutenbrunner C., Hildebrandt G., Moog R.), Lang, Frankfurt, pp. 113-120.

Hardeland R., Poeggeler B., Behrmann G., Fuhrberg B. (1996)

Enzymatic and non-enzymatic metabolism of methoxyindoles.

In: Metabolism and cellular dynamics of indoles.

(ed. by Hardeland R.), University of Göttingen, Göttingen, pp. 6-22. 


\section{Hardeland R., Poeggeler B., Burkhardt S., Zelosko V., Niebergall R., Tomás-Zapico}

C., Thuermann S., Schoenke M., Dose A., Gruetzner T., Coto-Montes A. (2003b)

Oxidation chemistry of melatonin: New aspects of radical reactions.

In: Neuroendocrine system and pineal gland with special reference to livestock.

(ed. by Haldar C., Singh S.S.), Banaras Hindu University, Varanasi, pp. 27-35.

\section{Hardeland R., Poeggeler B., Niebergall R., Zelosko V. (2003c)}

Oxidation of melatonin by carbonate radicals and chemiluminescence emitted during pyrrole ring cleavage.

J. Pineal Res. 34: 17-25.

\section{Hardeland R., Reiter R.J., Poeggeler B., Tan D.-X. (1993c)}

The significance of the metabolism of the neurohormone melatonin: antioxidative protection and formation of bioactive substances.

Neurosci. Biobehav. Rev. 17: 347-357.

\section{Hardeland R., Ressmeyer A.-R., Zelosko V., Burkhardt S., Poeggeler B. (2004)}

Metabolites of melatonin: Formation and properties of the methoxylated kynuramines AFMK and AMK.

In: Recent advances in endocrinology and reproduction: Evolutionary biotechnological and clinical implications.

(ed. by Haldar C., Singh S.S.), Banaras Hindu University, Varanasi, pp. 21-38.

\section{Hardeland R., Zelosko V., Niebergall R., Ressmeyer A.-R., Poeggeler B. (2003d)}

Interactions of carbonate radicals $\left(\mathrm{CO}_{3}{ }^{\circ}\right)$ with melatonin detected by chemiluminescence.

Clin. Lab. 49: 556.

\section{Hayaishi O. (1976)}

Properties and functions of indoleamine 2,3-dioxygenase.

J. Biochem. (Tokyo) 79: 13P-21P.

Helin-Salmivaara A., Huupponen R., Virtanen A., Klaukka T. (2005)

Adoptions of celecoxib and refecoxibit: a nationwide database study.

J. Clin. Pharm. Ther. 30: 145-152. 
Hirata F., Hayaishi O., Tokuyama T., Senoh S. (1974)

In vitro and in vivo formation of two new metabolites of melatonin.

J. Biol. Chem. 249: 1311-1313.

Huether G. (1993)

The contribution of extrapineal sites of melatonin synthesis to circulating melatonin levels in higher vertebrates.

Experientia 49: 665-670.

Huether G., Fettkötter I., Keilhoff G., Wolf G. (1997)

Serotonin acts as a radical scavenger and is oxidized to a dimer during the respiratory burst of activated microglia.

J. Neurochem. 69: 2096-2101.

Illnerova H. (1989)

Mechanism of the re-entrainment of the circadian rhythm in the rat pineal $N$-acetyltransferase activity to an eight-hour advance of the light-dark cycle: phase-jump is involved Brain Res. 494: 365-368

Illnerova H., Vanecek J. (1980)

Pineal rhythm in $\mathrm{N}$-acetyltransferase activity in rats under different artificial photoperiods and in natural daylight in the course of a year.

Neuroendocrinology 31: 321-326.

Kalgutkar A.S., Crews B.C., Rowlinson S.W., Marnett A.B., Kozak K.R., Remmel R.P., Marnett L.J. (2000)

Biochemically based design of cyclooxygenase-2 (COX-2) inhibitors: facile conversion of nonsteroidal anti-inflammatory drugs to potent and highly selective COX-2 inhibitors.

Proc. Natl. Acad. Sci. USA 97: 925-930.

Karasek M., Stawerskan R., Hilczer M., Zylinska K., Lewinski A. (2004)

Melatonin circadian rhythm in women with idiopathic hyperprolactinemia

Neuroendocrinol. Lett. 25: 411-414 
Kelly R.W., Amato F., Seamark R.F. (1984)

N-Acetyl-5-methoxy kynurenamine, a brain metabolite of melatonin, is a potent inhibitor of prostaglandin biosynthesis.

Biochem. Biophys. Res. Commun. 121: 372-379.

Kennaway D.J., Hugel H.M., Clarke S., Tjandra A., Johnson D.W., Royles P., Webb H.A., Carbone T. (1988)

Structure-activity studies of melatonin analogues in prepubertal male rats.

Aust. J. Biol. Sci. 41: 393-400.

Koolman J., Röhm K.-H. (1998)

Taschenatlas der Biochemie. 2. Aufl.

Thieme, Stuttgart.

Kopin I.J., Pare C.M.B., Axelrod J., Weissbach H. (1961)

The fate of melatonin in animals.

J. Biol. Chem. 236: 3072-3075.

Lerner A.B., Case J.D., Heinzelman R.V. (1959)

The structure of melatonin.

J. Am. Chem. Soc. 81: 6084-6085.

Lerner A.B., Case J.D., Takahashi Y. (1958)

Isolation of melatonin, a pineal factor that lightens melanocytes.

J. Am. Chem. Soc. 80: 2057-2058.

Levesque L.E., Brophy J.M., Zhang B. (2005)

The risk for myocardial infarctions with cyclooxygenase-2-inhibitors: a population study of elder adults.

Ann. Intern. Med. 142: 481-489. 
López-Burillo S., Tan X., Rodriguez-Gallego V., Manchester LC., Mayo J.C., Sainz R.M., Reiter R.J. (2003)

Melatonin and its derivatives cyclic 3-hydroxymelatonin, $\mathrm{N}^{1}$-acetyl- $\mathrm{N}^{2}$-formyl-5methoxykynuramine and 6-methoxymelatonin reduce oxidative DNA damage induced by Fenton reagents

J. Pineal Res. 34: 178-184.

Maestroni G.J.M. (1993)

The immunoneuroendocrine role of melatonin.

J. Pineal Res. 14: 1-10.

Manchester L.C., Poeggeler B., Alvares F.L., Ogden G.B., Reiter R.J. (1995)

Melatonin immunoreactivity in the photosynthetic procaryote, Rhodospirillum rubrum: Implications for an ancient antioxidant system.

Cell. Mol. Biol. Res. 41: 391-395.

Manchester L.C., Tan D.-X., Reiter R.J., Park W., Monis K., Qi W. (2000)

High levels of melatonin in the seeds of edible plants: possible function in germ tissue protection.

Life Sci. 67: 3023-3029.

Martinez G.R., Almeida E.A., Klitzke C.F., Onuki J., Prado F.M., Medeiros M.H., Di Mascio P. (2005)

Measurement of melatonin and its metabolites: importance for the evaluation of their biological roles.

Endocrine 27: 111-118

Matsubara E., Bryant-Thomas T., Pacheco Quinto J., Henry T.L., Poeggeler B., Herbert D., Cruz-Sanchez F., Chyan Y.J., Smith M.A., Perry G., Shoji M., Abe K., Leone A., Grundke-Ikbal I., Wilson G.L., Ghiso J., Williams C., Refolo L.M., Pappolla M.A., Chain D.G., Neria E. (2003)

Melatonin increases survival and inhibits oxidative and amyloid pathology in a transgenic model of Alzheimer's disease.

J. Neurochem. 85: 1101-1108. 
Mayo J.C., Sainz R.M., Tan D.-X., Hardeland R., Leon J., Rodriguez C., Reiter R.J. (2005)

Anti-inflammatory actions of melatonin and its metabolites, $\mathrm{N}^{1}$-acetyl- $\mathrm{N}^{2}$-formyl-5methoxykynuramine (AFMK) and $\mathrm{N}^{1}$-acetyl-5-methoxykynuramine (AMK), in macrophages. J. Neuroimmunol. 165: 139-149.

McCord C.P., Allen F.P. (1917)

Evidences associating pineal gland function with alterations in pigmentation.

J. Exp. Zool. 23: 207-224.

Messner M., Hardeland R., Rodenbeck A., Huether G. (1998)

Tissue retention and subcellular distribution of continuously infused melatonin in rats under near physiological conditions.

J. Pineal Res. 25: 251-259.

Mor M., Silva C., Vacondio F., Plazzi P., Bertoni S., Spadoni G., Diamantini G., Bedini A., Tarzia G., Zusso M. Fraschini F., Giusti P. (2004)

Indole-based analogs of melatonin: in vitro antioxidant and cytoprotective activities.

J. Pineal Res. 36: 95-102.

Pappolla M.A., Omar R.A., Chyan Y.-J., Ghiso J., Hsiao K., Bozner P., Smith M., Cruz-Sanchez F. (2001)

Induction of NADPH cytochrome P450 reductase by the Alzheimer $\beta$-protein. Amyloid as a "foreign body".

J. Neurochem. 78: 121-128.

Pardridge, W.M. and Mietus, L.J. (1980).

Transport of albumin-bound melatonin through the blood-brain barrier.

J. Neurochem. 34: 1761-1763.

Pereira C., Santos M.S., Oliveira C. (1998)

Mitochondrial function impairment induced by amyloid peptide on PC12 cells.

Neuroreport 9: 1749-1755. 


\section{Pereira C., Santos M.S., Oliveira C. (1999)}

Involvement of oxidative stress on the impairment of energy metabolism induced by $A \beta$ peptides on PC12 cells: protection by antioxidants.

Neurobiol. Dis. 6: 209-219.

Poeggeler B., Balzer I., Hardeland R., Lerchl A. (1991)

Pineal hormone melatonin oscillates also in the dinoflagellate Gonyaulax polyedra.

Naturwissenschaften 78: 268-269.

Poeggeler B., Miravalle L., Zagorski M.G., Wisniewski T., Chyan Y.J., Zhang Y., Shao H., Bryant-Thomas T., Vidal R., Frangione B., Ghiso J., Pappolla M.A. (2001)

Melatonin reverses the profibrillogenetic activity of apolipoportein E4 on the Alzheimer amyloid $A \beta$ peptide.

Biochemistry 40: 14995-15001.

Poeggeler B., Thuermann S., Dose A., Schoenke M., Burkhardt S., Hardeland R. (2002)

Melatonin's unique radical scavenging properties - Roles of its functional substituents as revealed by a comparison with its structural analogs.

J. Pineal Res. 33: 20-30.

Re R., Pellegrini N., Proteggente A., Pannals A., Yang M., Rice-Evans C. (1999) Antioxidant activity applying an improved ABTS cation radical decolorization assay. Free Radic. Biol. Med. 26: 1231-1237.

Reiter R.J. (1991)

Pineal melatonin: Cell biology of its synthesis and of its physiological interactions.

Endocr. Rev. 12: 151-180.

Reiter R.J. (1998)

Oxidative damage in the central nervous system: Protection by melatonin.

Prog. Neurobiol. 56: 359-384. 
Reiter R.J., Tan D.-X. (2002)

Role of CSF in the transport of melatonin.

J. Pineal Res. 33: 61.

Reiter R.J., Tan D.-X., Burkhardt S., Manchester L.C. (2001)

Melatonin in plants.

Nutr. Rev. 59: 286-290.

Reiter R.J., Tan D.-X., Burkhardt S. (2002)

Reactive oxygen and nitrogen species and cellular and organismal decline: Amelioration with melatonin.

Mech. Ageing Dev., 123: 1007-1019.

Ressmeyer A.-R. (2002)

Untersuchungen über den Melatonin-Metaboliten $\mathrm{N}^{1}$-Acetyl-5-methoxykynuramin (AMK).

Diplomarbeit, Göttingen.

Ressmeyer A.-R., Mayo J.C., Zelosko V., Sáinz R.M., Tan D.-X., Poeggeler B., Antolín I., Zsizsik B.K., Reiter R.J., Hardeland R. (2003)

Antioxidant properties of the melatonin metabolite $\mathrm{N}^{1}$-acetyl-5-methoxykynuramine (AMK): scavenging of free radicals and prevention of protein destruction.

Redox Rep. 8: 205-213.

Rodrigues M.R., Rodríguez D., Catalani L.H., Russo M., Campa A. (2003)

Interferon-gamma independent oxidation of melatonin by macrophages.

J. Pineal Res. 34: 69-74.

Rosen J., Than N.N., Koch D., Poeggeler B., Laatsch H., Hardeland R. (2006)

Interactions of melatonin and its metabolites with the ABTS cation radical: extension of the radical scavenger cascade and formation of a novel class of oxidation products, C2-substituted 3-indolinones

J. Pineal Res. 41: 374-381

Sainz R.M., Mayo J.C., Reiter R.J., Tan D.-X., Rodriguez C. (2003)

Apoptosis in primary lymphoid organs with aging.

Microsc. Res. Tech. 62: 524-539. 
Schmidt S.I. (2004)

Redox-Reaktionen des Melatonin-Metaboliten AMK ( $\mathrm{N}^{1}$-Acetyl-5-methoxykynuramin). Staatsexamensarbeit, Göttingen.

Schoenke M., Poeggeler B., Burkhardt S., Hardeland R., Zsizsik B.K. (2001)

A comparative study on products formed from indoleamines in radical-generating systems.

In: Actions and redox properties of melatonin and other aromatic amino acid metabolites. (ed. by Hardeland R.), Cuvillier, Göttingen, pp. 32-38.

Silva S.O., Rodrigues M.R., Carvalho S.R.Q., Catalani L.H., Campa A., Ximenes V.F. (2004)

Oxidation of melatonin and its catabolites, $\mathrm{N}^{1}$-acetyl- $\mathrm{N}^{2}$-formyl-5-methoxykynuramine and $\mathrm{N}^{1}$-acetyl-5-methoxykynuramine, by activated leucocytes.

J. Pineal Res. 37: 171-175.

Skwarlo-Sonta K. (2002)

Melatonin in immunity: comparative aspects.

Neuroendocrinol. Lett. 23, Suppl 1: 61-66.

Slominski A., Pisarchik A., Semak I., Sweatman T., Wortsman J., Szczesniewski A., Slugocki G., McNulty J., Kauser S., Tobin D.J., Jing C., Johansson O. (2002)

Serotoninergetic and melatoninergetic systems are fully expressed in human skin.

FASEB J. 16: 896-898.

Slominski A., Wortsman J., Tobin D.J. (2005)

The cutaneous serotoninergic/melatoninergic system: securing a place under the sun.

FASEB J. 19: 176-194.

Spadoni G., Balsamini C., Bedini A., Diamantini G., Di Giacomo B., Tontini A., Tarzia G., Mor M., Plazzi P., Rivara S., Nonno R., Pannacci M., Lucini V., Fraschini F., Stankov BM. (1998)

2-[N-acylamino(C1-C3)alkyl]indoles as MT1 melatonin receptor partial agonists, antagonists and putative inverse agonists.

J. Med. Chem. 41:3624-3634. 
Spadoni G., Balsamini C., Diamantini G., Tontini A., Tarzia G., Mor M., Rivara S., Plazzi P., Nonno R., Lucini V., Pannacci M., Fraschini F., Stankov BM. (2001)

2-N-acylaminoalkylindoles: design and quantitative structure-activity relationship studies leading to MT2-selective melatonin antagonists.

J. Med. Chem. 44:2900-2912.

Spadoni G., Diamantini G., Bedini A., Tarzia G., Vacondio F., Silva C., Rivara M., Mor M., Plazzi P., Zusso M., Franceschini F., Giusti P. (2006)

Synthesis, antioxidant activity and structure-activity relationships for a new series of 2-(Nacylaminoethyl) indoles with melatonin-like cytoprotective activity.

J. Pineal Res. 40:259-269.

Sprenger J., Hardeland R. (1999)

Melatonin and 5-methoxytryptamine in yeast: Requirement of precursors.

In: Studies on antioxidants and their metabolites.

(ed. by Hardeland R.), Cuvillier, Göttingen, pp: 191-198.

Sugden D (1989)

Melatonin biosynthesis in the mammalian pineal gland

Experientia 45: 922-932.

Tan D.-X. (2002)

Interrelationships between melatonin and oxidative stress: Mechanistic studies of the reactions of melatonin and its metabolites with free radicals.

Cuvillier, Göttingen.

Tan D.-X., Chen L.D., Poeggeler B., Manchester L.C., Reiter R.J. (1993)

Melatonin: a potent, endogenous hydroxyl radical scavenger.

Endocr. J. 1: 57-60. 
Tan D.-X., Manchester L.C., Burkhardt S., Saínz R.M., Mayo J.C., Kohen R., Shohami E., Huo Y.-S., Hardeland R., Reiter R.J. (2001)

$\mathrm{N}^{1}$-acetyl- $\mathrm{N}^{2}$-formyl-5-methoxykynuramine, a biogenic amine and melatonin metabolite, functions as a potent antioxidant.

FASEB J. 15: 2294-2296.

Tan D.-X., Reiter R.J., Manchester L.C., Yan M.-T., El-Sawi M., Sainz R.M., Mayo J.C., Kohen R., Allegra M., Hardeland R. (2002)

Chemical and physical properties and potential mechanisms: Melatonin as a broad spectrum antioxidant and free radical scavenger.

Curr. Top. Med. Chem. 2: 181-197.

Tan D.-X., Hardeland R., Manchester L.C., Poeggeler B., Lopez-Burillo S., Mayo

J.C., Sainz R.M., Reiter R.J. (2003a)

Mechanistic and comparative studies of melatonin and classic antioxidants in terms of their interactions with the ABTS cation radical.

J. Pineal Res. 34: 249-259.

Tan D.-X., Manchester L.C., Hardeland R., Lopez-Burillo S., Mayo J.C., Sainz R.M., Reiter R.J. (2003b)

Melatonin: a hormone, a tissue factor, an autocoid, a paracoid, and an antioxidant vitamin. J. Pineal Res. 34: 75-78.

Tesoriere L., Avellone G., Ceraulo L., D’Arpa D., Allegra M., Livrea M.A. (2001)

Oxidation of melatonin by oxoferryl hemoglobin: a mechanistic study.

Free Radic. Res. 35: 633-642.

Than N.N., Heer C., Laatsch H., Hardeland R. (2006)

Reactions of the melatonin metabolite $\mathrm{N}^{1}$-acetyl-5-methoxykynuramine (AMK) with the ABTS cation radical: identification of new oxidation products.

Redox Rep. 11: 15-24 


\section{Tomás-Zapico C., Hardeland R., Poeggeler B., Coto-Montes A. (2001)}

3-Hydroxyanthranilic acid and 3-hydroxykynurenine as photooxidants: catalysis of $\mathrm{N}^{1}$-acetyl$\mathrm{N}^{2}$-formyl-5-methoxykynuramine formation from melatonin.

In: Actions and redox properties of melatonin and other aromatic amino acid metabolites.

(ed. by Hardeland R.), Cuvillier, Göttingen, pp. 136-141.

Tricoire H., Locatelli A., Chemineau P., Malpaux B. (2002)

Melatonin enters the cerebrospinal fluid through the pineal recess.

Endocrinology 143: 84-90.

Uría H., Fuhrberg B., Poeggeler B., Hardeland R., Menéndez-Peláez A. (1994)

Photooxidation of melatonin.

In: Cell biological problems in chronobiology.

(ed. by Hardeland R.), University of Göttingen, Göttingen, pp. 89-99.

\section{Uría H., Hardeland R., Menéndez-Peláez A., Fuhrberg B. (1995)}

Extracts of the Syrian hamster Harderian gland catalyse photooxidation of melatonin.

In: Cellular rhythms and indoleamines.

(ed. by Hardeland R.), University of Göttingen, Göttingen, pp. 140-144.

\section{Waldhauser F., Ehrhart B., Forster E. (1993)}

Clincal aspect of the melatonin action: impact of development, aging, and puberty, involvement of melatonin in psychiatric disease and importance of neuroimmunoendocrine interactions.

Experientia 49: 671-681

Walton L.J., Franklin I.J., Bayston L.C., Brown L.C., Greenhalgh R.M., Taylor G.W., Powell J.T. (1999)

Inhibition of prostaglandin E2 synthesis in abdominal aortic aneurysms: implication for smooth muscle cell viability, inflammatory process, and the expansion of abdominal aortic aneurysms.

Circulation 100: 48-54. 
Weishaupt J.H., Bartels C., Pölking E., Dietrich J., Rohde G., Poeggeler B., Mertens N., Sperling S., Bohn M., Hüther G., Schneider A., Bach A., Sirén A.L., Hardeland R., Bähr M., Nave K.A., Ehrenreich H. (2006)

Reduced oxidative damage in ALS by high-dose enteral melatonin treatment.

J. Pineal Res. 41: 313-321

Ximenes V.F., Catalani L.H., Campa A. (2001)

Oxidation of melatonin and tryptophan by an HRP cycle involving compound III.

Biochem. Biophys. Res. Commun. 287: 130-134. 
Joachim Rosen

Valentinsbreite 13

37077 Göttingen

\section{Persönliche Daten:}

Name

Anschrift

geboren am

Staatsangehörigkeit

Schulbildung:

1967-1971

$1971-1972$

1972-1982

Berufsausbildung:

1982-1985

1988-1999

1999-2001

2002-2006
Joachim Rosen

Valentinsbreite 13

37077 Göttingen

11.01.1961 in Lingen/Emsland

Deutsch

Franziskus-Demann Schule in Freren

Maristen-Gymnasium St. Josef in Meppen

Gymnasium Georgianum in Lingen/Emsland

Studium der Medizin an der Medizinischen Hochschule Hannover (MHH)

Studium der Biologie an der Georg-August-Universität Göttingen.

Diplomand in der Inneren Medizin, Abteilung Hämatologie und Onkologie der Georg-August-Universität. Thema: Untersuchung zur Abspaltung der Extrazellulären Domäne der DiscoidinDomain-Rezeptortyrosinkinase DDR1.

Doktorand im Institut für Zoologie und Anthropologie, Abteilung Stoffwechselphysiologie der Georg-AugustUniversität Göttingen. Thema: Vergleich der Radikalreaktionen von Melatonin und deren strukturverwandten Indolaminen in unterschiedlichen Oxidationssystemen. 